Florida International University FIU Digital Commons

6-30-2015

\title{
Intraoperative Guidance for Pediatric Brain Surgery based on Optical Techniques
}

Yinchen Song

Florida International University, songyinchen@gmail.com

DOI: $10.25148 /$ etd.FIDC000092

Follow this and additional works at: https://digitalcommons.fiu.edu/etd

Part of the Bioimaging and Biomedical Optics Commons, and the Biomedical Devices and Instrumentation Commons

\section{Recommended Citation}

Song, Yinchen, "Intraoperative Guidance for Pediatric Brain Surgery based on Optical Techniques" (2015). FIU Electronic Theses and Dissertations. 2207.

https://digitalcommons.fiu.edu/etd/2207

This work is brought to you for free and open access by the University Graduate School at FIU Digital Commons. It has been accepted for inclusion in FIU Electronic Theses and Dissertations by an authorized administrator of FIU Digital Commons. For more information, please contact dcc@fiu.edu. 


\section{FLORIDA INTERNATIONAL UNIVERSITY}

Miami, Florida

\section{INTRAOPERATIVE GUIDANCE FOR PEDIATRIC BRAIN SURGERY BASED ON OPTICAL TECHNIQUES}

A dissertation submitted in partial fulfillment of the requirements for the degree of DOCTOR OF PHILOSOPHY

in

BIOMEDICAL ENGINEERING

by

Yinchen Song 
To: Dean Amir Mirmiran

College of Engineering and Computing

This dissertation, written by Yinchen Song, and entitled Intraoperative Guidance for Pediatric Brain Surgery based on Optical Techniques, having been approved in respect to style and intellectual content, is referred to you for judgment.

We have read this dissertation and recommend that it be approved.

Anthony McGoron

Malek Adjouadi

Armando Barreto

Jorge J. Riera, Co-Major Professor

Wei-Chiang Lin, Co-Major Professor

Date of Defense: June 30, 2015

The dissertation of Yinchen Song is approved.

$\begin{array}{r}\text { Dean Amir Mirmiran } \\ \text { College of Engineering and Computing } \\ \hline \begin{array}{r}\text { Dean Lakshmi N. Reddi } \\ \text { University Graduate School }\end{array}\end{array}$

Florida International University, 2015 
C Copyright 2015 by Yinchen Song

All rights reserved. 


\section{ACKNOWLEDGMENTS}

I sincerely appreciate the guidance and patience from my major professor Dr. WeiChiang Lin and co-major professor Dr. Jorge J. Riera, who led me to complete this Ph.D. dissertation. Dr. Lin and Dr. Riera's mentorship enlightened my view of biomedical research and assisted me to overcome the difficulties in my studies and my life. I could not accomplish this dissertation without the guidance from my dissertation committee, Dr. Anthony McGoron, Dr. Malek Adjouadi, and Dr. Armando Barreto. I would like to give thanks to my lab members, Dr. Po-Ching Chen, Dr. Nitin Yadav, Dr. Jihye Bae, Rafael A. Torres, Sarahy Garcia, Yisel Frometa, Abhay Deshmukh, Lakshmini Balachandar, Mohammad Soltani, Mohamed Almadi, Jared Leichner, and Arnold Joasil for their assistance and supports in the research projects. I would also like to acknowledge the assistance I received from Dr. Jessica Ramella-Roman for the phantom calibration studies. Thanks also to Dr. Fahmeed Hyder from Yale University and Dr. Ying Zheng from University of Reading for providing the insightful suggestions to my research projects.

I would like to acknowledge the financial support I received throughout my Ph.D. study, including the Teaching Assistantship from the Department of Biomedical Engineering and Research Assistantship from the Nicklaus Children's Hospital and National Institute of Health.

My gratitude also goes to all my friends who helped me all of these years and made my stay at Miami much more pleasurable. Finally, I would like to express my gratitude to my parents, my grandma, and the rest of my family for their unconditional love and support. 


\title{
ABSTRACT OF THE DISSERTATION \\ INTRAOPERATIVE GUIDANCE FOR PEDIATRIC BRAIN SURGERY BASED ON \\ OPTICAL TECHNIQUES
}

\author{
by \\ Yinchen Song \\ Florida International University, 2015 \\ Miami, Florida

\section{Professor Wei-Chiang Lin, Co-Major Professor \\ Professor Jorge J. Riera, Co-Major Professor}

For most of the patients with brain tumors and/or epilepsy, surgical resection of brain lesions, when applicable, remains one of the optimal treatment options. The success of the surgery hinges on accurate demarcation of neoplastic and epileptogenic brain tissue. The primary goal of this $\mathrm{PhD}$ dissertation is to demonstrate the feasibility of using various optical techniques in conjunction with sophisticated signal processing algorithms to differentiate brain tumor and epileptogenic cortex from normal brain tissue intraoperatively.

In this dissertation, a new tissue differentiation algorithm was developed to detect brain tumors in vivo using a probe-based diffuse reflectance spectroscopy system. The system as well as the algorithm were validated experimentally on 20 pediatric patients undergoing brain tumor surgery at Nicklaus Children's Hospital. Based on the three indicative parameters, which reflect hemodynamic and structural characteristics, the new algorithm was able to differentiate brain tumors from the normal brain with a very high accuracy. 
The main drawbacks of the probe-based system were its high susceptibility to artifacts induced by hand motion and its interference to the surgical procedure. Therefore, a new optical measurement scheme and its companion spectral interpretation algorithm were devised. The new measurement scheme was evaluated both theoretically with Monte Carlo simulation and experimentally using optical phantoms, which confirms the system is capable of consistently acquiring total diffuse reflectance spectra and accurately converting them to the ratio of reduced scattering coefficient to absorption coefficient $\left(\mu_{\mathrm{s}}{ }^{\prime}(\lambda) / \mu_{\mathrm{a}}(\lambda)\right)$. The spectral interpretation algorithm for $\mu_{\mathrm{s}}{ }^{\prime}(\lambda) / \mu_{\mathrm{a}}(\lambda)$ was also validated based on Monte Carlo simulation. In addition, it has been demonstrated that the new measurement scheme and the spectral interpretation algorithm together are capable of detecting significant hemodynamic and scattering variations from the Wistar rats' somatosensory cortex under forepaw stimulation.

Finally, the feasibility of using dynamic intrinsic optical imaging to distinguish epileptogenic and normal cortex was validated in an in vivo study involving 11 pediatric patients with intractable epilepsy. Novel data analysis methods were devised and applied to the data from the study; identification of the epileptogenic cortex was achieved with a high accuracy. 


\section{TABLE OF CONTENTS}

CHAPTER

PAGE

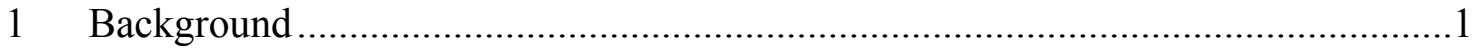

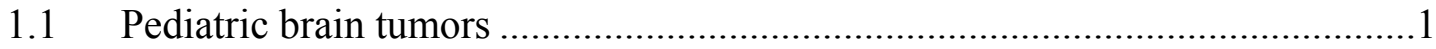

1.2 Pediatric epilepsy …………………………….........................................

1.3 Intraoperative guidance for pediatric brain surgery .......................................

1.3.1 Frameless stereotaxic neuronavigation...............................................

1.3.2 Intraoperative magnetic resonance imaging ..............................................

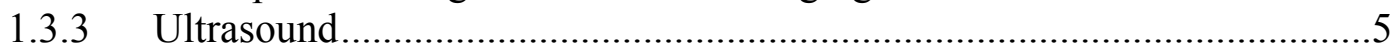

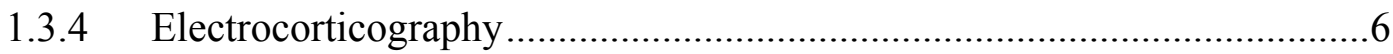

1.3.5 Diffuse reflectance spectroscopy ……………........................................6

1.3.6 Dynamic intrinsic optical imaging ..........................................................

2 Significance and goals ...............................................................................

3 Intraoperative pediatric brain tumor detection using diffuse reflectance

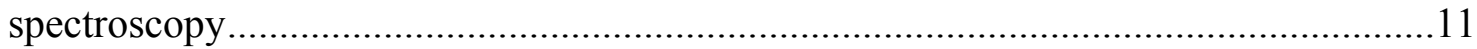

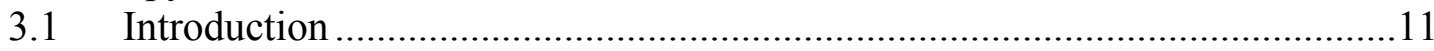

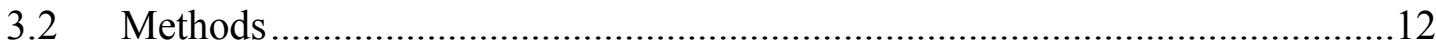

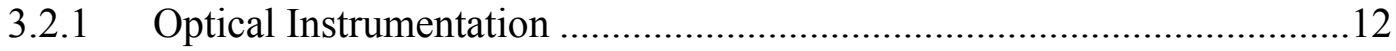

3.2.2 Clinical data acquisition..................................................................13

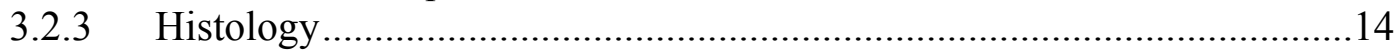

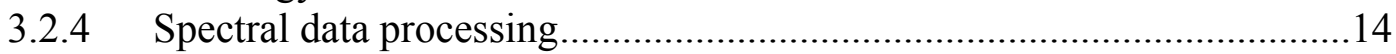

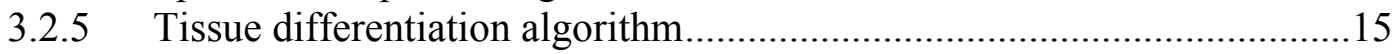

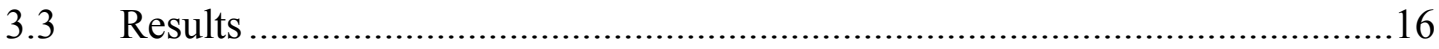

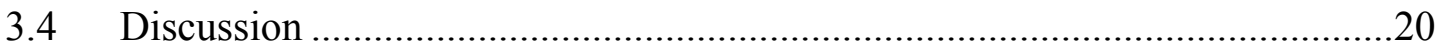

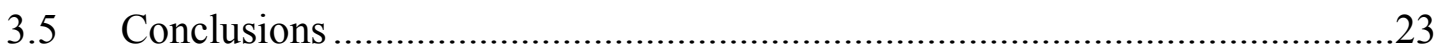

4 Determination of the optical properties of turbid media using total diffuse

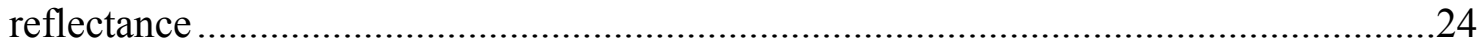

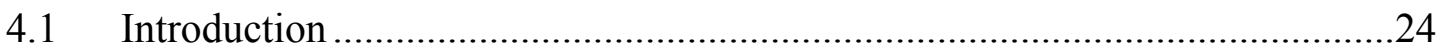

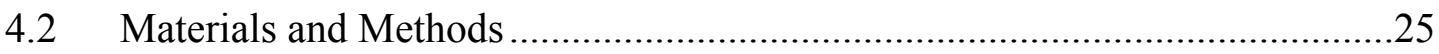

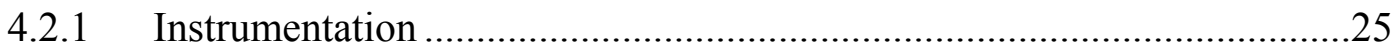

4.2.2 Look-up table for $\mu_{\mathrm{s}}{ }^{\prime} / \mu_{\mathrm{a}}$ estimation based on total diffuse

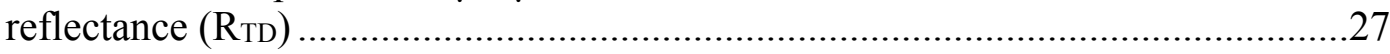

4.2.3 Evaluation of the accuracy of the look-up table using Monte Carlo

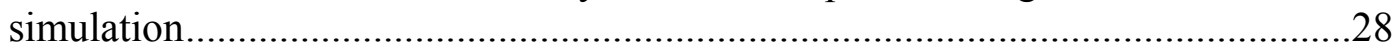

4.2.4 Evaluation of point spectroscopic detection modality of the hybrid

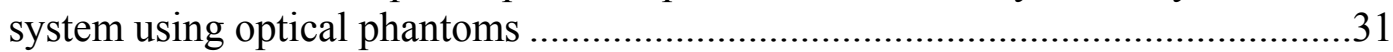

4.2.5 Evaluation of the imaging modality of the hybrid system using

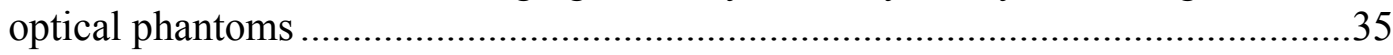

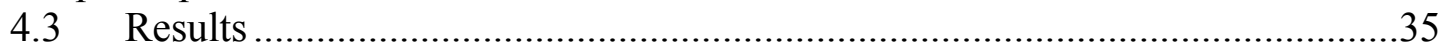




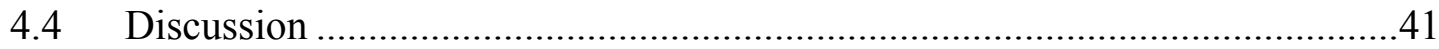

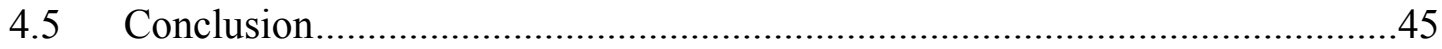

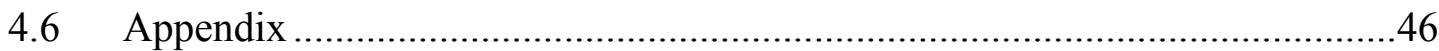

5 A new algorithm for determination of hemodynamic and structural characteristics of brain tissue from total diffuse reflectance measurements...................49

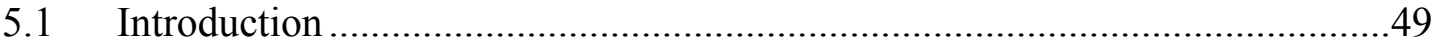

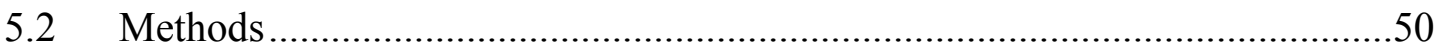

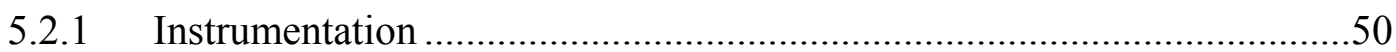

5.2.2 Spectral interpretation algorithm ..........................................................51

5.2.3 Validation based on Monte Carlo simulation ……………………….........55

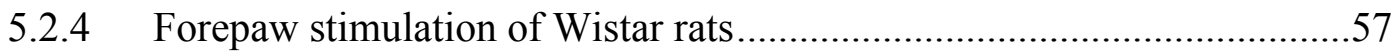

5.2.5 Acquisition of point spectroscopic detection modality .............................59

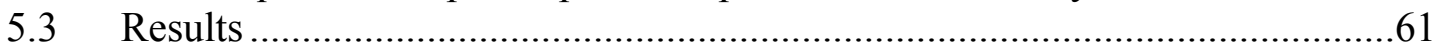

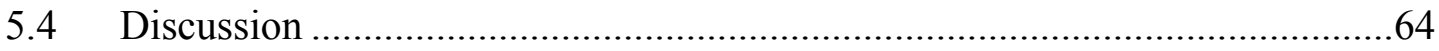

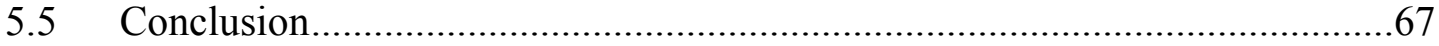

6 Intraoperative optical mapping of epileptogenic cortices in pediatric patients ......68

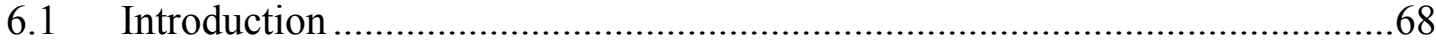

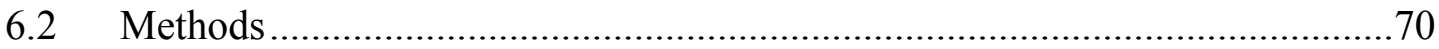

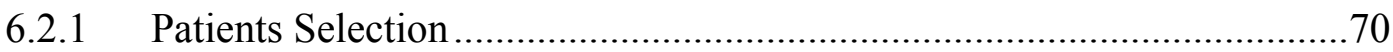

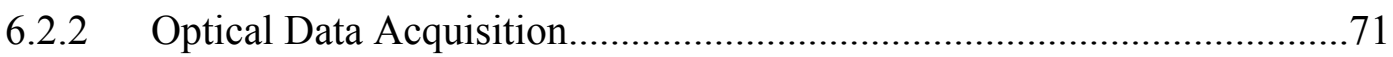

6.2.3 Optical Data Analysis (Method 1) ...........................................................

6.2.4 Electrocorticography (ECoG) Acquisition and Analysis............................75

6.2.5 Stochastic modeling and machine learning (Method 2) …….....................76

6.2.6 Static Digital Imaging and Analysis of the Vasculature

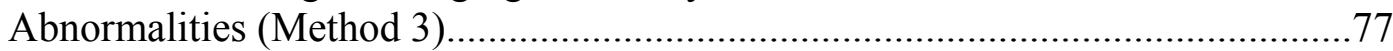

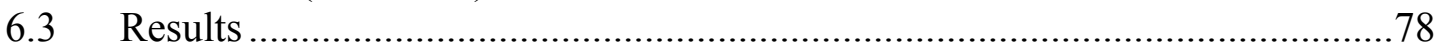

6.3.1 Correlation, clustering and Granger causality (Method 1) ........................79

6.3.2 Stochastic modeling and machine learning (Method 2) ............................83

6.3.3 Vasculature abnormalities in the superficial layer (Method 3)..................84

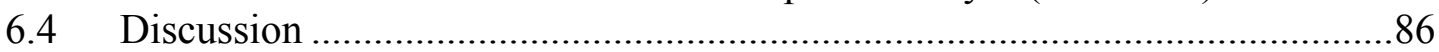

6.4.1 Hemodynamic low-frequency oscillations (LFOs).....................................86

6.4.2 Stochastic modeling to characterize the underlying

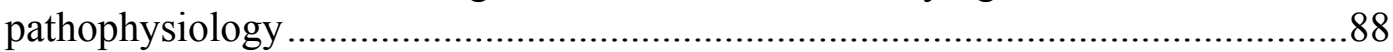

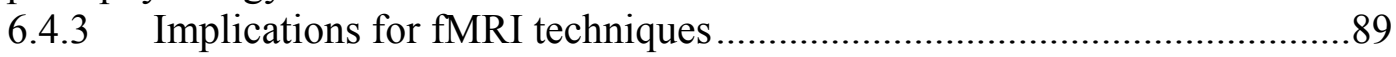

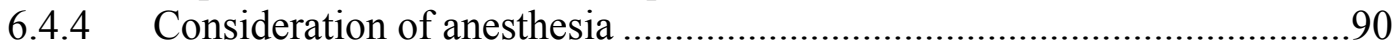

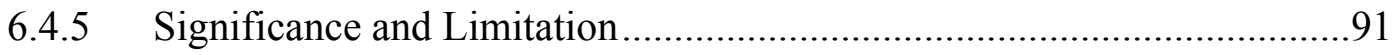

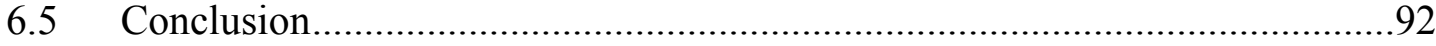

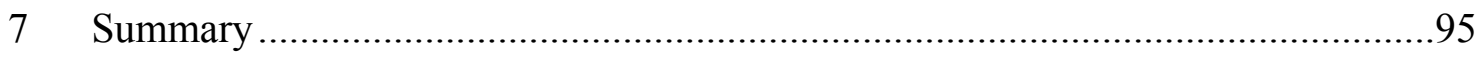

LIST OF REFERENCES ..................................................................................98

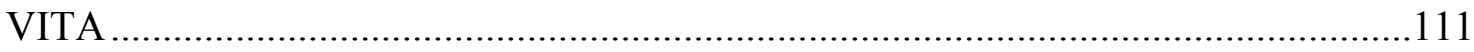




\section{LIST OF TABLES}

TABLE

PAGE

Table 3. 1 Demographic data on the twenty patients who participated in the clinical study

Table 3. 2 Tissue differentiation accuracy, sensitivity, and specificity for three individual classification criteria and four combined classification criterion with support vector machine. 20

Table 4. 1 Ranges of $\mu_{\mathrm{s}}$ ' $/ \mu_{\mathrm{a}}$ used in look-up table building 28

Table 5. 1 Ranges of parameters used in the Monte Carlo simulation to generate $\mathrm{R}_{\mathrm{TD}}$ spectra 56

Table 6. 1 Patients' demographic and clinical information. .71

Table 6. 2 Analysis Summary 82

Supplementary Table 6. 1 Surgery-related Information 


\section{LIST OF FIGURES}

FIGURE

PAGE

Figure 3. 1 Histograms of (a) $\mathrm{n}[\mathrm{Hb}]$, (b) nRd700and (c) $\mathrm{nSatO}_{2}$ from normal and neoplastic brain tissue.

Figure 3. 2 Scatter plot of (a) $n[\mathrm{Hb}]$ and $n R d 700$, (b) $n[\mathrm{Hb}]$ and $n \mathrm{natO}_{2}$, and (c) $\mathrm{nRd} 700$ and $\mathrm{nSatO}_{2}$ from normal cortex and brain tumor.

Figure 3. 3 The distribution of tumor and normal tissue sites, in accordance with their corresponding $\mathrm{n}[\mathrm{Hb}], \mathrm{nRd} 700$, andnSatO ${ }_{2}$. The black hyperplane that differentiate brain tumors from normal cortex was obtained from support vector machine based on all three normalized indicative parameters. The performance of the differentiation is shown in Table 3.2.

Figure 4. 1 The schematic of the hybrid spectroscopy imaging system.

Figure 4. 2 Relationships between total diffuse reflectance RTD and $\mu_{\mathrm{s}}{ }^{\prime} / \mu_{\mathrm{a}}$. Black dots are RTD derived from the Monte Carlo simulations. The blue line is the fitted curve obtained using the Curve Fitting Toolbox of Matlab.

Figure 4. 3 Experimental setup for evaluating the function of the spectroscopic point-detection of the hybrid system. The flow chart on the left shows the measurement procedure.

Figure 4. 4 Experimental setup for evaluating the function of the spectroscopic point-detection of the hybrid system. The flow chart on the top shows the measurement procedure.

Figure $4.5 \mathrm{~A})$ Absolute percentage error $|\Delta \mathrm{E}|$ in $\mu_{\mathrm{s}}{ }^{\prime} / \mu_{\mathrm{a}}$ estimations as a function of $\mathrm{R}_{\mathrm{TD}}$ and anisotropy factor (g). B) Absolute percentage error $|\Delta \mathrm{E}|$ in $\mathrm{R}_{\mathrm{TD}}$ estimations as a function of $\mu_{\mathrm{s}}{ }^{\prime} / \mu_{\mathrm{a}}$ and g. The look-up table of $\mu_{\mathrm{s}} / \mu_{\mathrm{a}}$ verse $\mathrm{R}_{\mathrm{TD}}$ with $\mathrm{g}=0.85$ was used for the conversion process, and $|\Delta \mathrm{E}|$ was calculated using Eq. (4.1).

Figure 4. $6 \mathrm{~A})$ Absolute percentage error $|\Delta \mathrm{E}|$ in $\mu_{\mathrm{s}}{ }^{\prime} / \mu_{\mathrm{a}}$ estimations as a function of $\mathrm{R}_{\mathrm{TD}}$ and refractive index (n). B) Absolute percentage error $|\Delta \mathrm{E}|$ in $\mathrm{R}_{\mathrm{TD}}$ estimations as a function of $\mu_{\mathrm{s}}{ }^{\prime} / \mu_{\mathrm{a}}$ and $\mathrm{n}$. The look-up table of $\mu_{\mathrm{s}} / \mu_{\mathrm{a}}$ verse $\mathrm{R}_{\mathrm{TD}}$ with $\mathrm{n}=1.3$ was used in al all conversion processes, and $|\Delta \mathrm{E}|$ was calculated using Eq. (4.1).

Figure 4. 7 A) Absolute percentage error $|\Delta \mathrm{E}|$ in $\mu_{\mathrm{s}}{ }^{\prime} / \mu_{\mathrm{a}}$ estimations and B) correlation coefficients of the linear fitting between the input and the 
estimated $\mu_{\mathrm{s}}{ }^{\prime} / \mu_{\mathrm{a}}$ values under four different conditions of $\mathrm{n}$ and $\mathrm{g}$, over the range of $\mu_{\mathrm{s}}{ }^{\prime} / \mu_{\mathrm{a}}$ depicted in Table 4.1

Figure 4. 8 Absolute percentage error $|\Delta \mathrm{E}|$ in $\mu_{\mathrm{s}}{ }^{\prime}(\lambda) / \mu_{\mathrm{a}}(\lambda)$ estimations using $\mathrm{R}_{\mathrm{TD} \_ \text {eva }}(\lambda)$ between $500 \mathrm{~nm}$ and $640 \mathrm{~nm}$. The references used are $\mu_{\mathrm{s}}{ }^{\prime}(\lambda) / \mu_{\mathrm{a}}(\lambda)$ of the optical phantoms determined by the double integrating sphere technique. The middle line represents the mean $|\Delta \mathrm{E}|$ calculated from all 8 phantoms; the error bars the standard deviation. One optical phantom was used as the reference standard in this study.

Figure 4. 9 Representative results from the experimental evaluation study of the point spectroscopic detection modality of the hybrid system. An optical phantom was used as the reference standard in this study. A) $R_{\text {TD_eva }}(\lambda)$ verse $\mathrm{RTD}_{\mathrm{TD} \text { theory }}(\lambda)$ at $\lambda=633 \mathrm{~nm}$. B) Measured $\mu_{\mathrm{s}}{ }^{\prime}(\lambda) / \mu_{\mathrm{a}}(\lambda)$ verse theoretical $\mu_{\mathrm{s}}{ }^{\prime}(\lambda) / \mu_{\mathrm{a}}(\lambda)$ at $\lambda=633 \mathrm{~nm}$. The solid lines in the figures are the linear regression lines

Figure 4. 10 Absolute percentage error $|\Delta \mathrm{E}|$ of $\mu_{\mathrm{s}}{ }^{\prime}(\lambda) / \mu_{\mathrm{a}}(\lambda)$ estimations between $500 \mathrm{~nm}$ and $640 \mathrm{~nm}$. The references used are $\mu_{\mathrm{s}}{ }^{\prime} / \mu_{\mathrm{a}}$ of the optical phantoms determined by the double integrating sphere technique. The middle line represents the mean $|\Delta \mathrm{E}|$ calculated from all 8 phantoms; the error bar the standard deviation. A paper-based reflectance material was used as the reference standard in this study

Figure 4. 11 Representative results from the evaluation study of the point spectroscopic detection modality of the hybrid system. A paper-based reflectance material was used as the reference standard in this study. A) $\mathrm{R}_{\mathrm{TD} \_ \text {eva }}(\lambda)$ verse $\mathrm{R}_{\mathrm{TD} \text { _theory }}(\lambda)$ at $\lambda=633 \mathrm{~nm}$. B) Measured $\mu_{\mathrm{s}}{ }^{\prime}(\lambda) / \mu_{\mathrm{a}}(\lambda)$ verse theoretical $\mu_{\mathrm{s}}{ }^{\prime}(\lambda) / \mu_{\mathrm{a}}(\lambda)$ at $\lambda=633 \mathrm{~nm}$. The solid lines in the figures are the linear regression lines.

Figure 4. 12 Results from the evaluation study of the imaging modality of the hybrid system. An optical phantom was used as the reference standard in this study. A) $R_{T D}$ eva $(\lambda)$ verse $\operatorname{R}_{\text {TD_theory }}(\lambda)$ at $\lambda=500 \mathrm{~nm}$. B) Measured $\mu_{\mathrm{s}}{ }^{\prime}(\lambda) / \mu_{\mathrm{a}}(\lambda)$ verse theoretical $\mu_{\mathrm{s}}{ }^{\prime}(\lambda) / \mu_{\mathrm{a}}(\lambda)$ at $\lambda=500 \mathrm{~nm}$. The solid lines in the figures are the linear regression lines.

Figure 4. 13 Diffuse reflectance $\left(\mathrm{R}_{\mathrm{d}}\right)$ as a function of radius $(\mathrm{r})$ at three different wavelengths (A-500 nm, B-600 nm, and C-700 nm). The red curve demonstrated the narrowest diffuse reflectance profile, while the blue one is the widest one. The dash lines represent the cutoffs within which $95 \%$ of total diffuse reflectance is observed. To generate these $R_{d}(r)$, the Monte Carlo simulation was carried out with the optical properties of human brain [94]. Specifically, $\mathrm{n}$ is $1.395, \mathrm{~g}$ is $0.88, \mu_{\mathrm{a}}$ is $1.6-4.5 \mathrm{~cm}^{-1}$ at $500 \mathrm{~nm}, 0.6-1.7$ 
$\mathrm{cm}^{-1}$ at $600 \mathrm{~nm}$ and $0.07-0.19 \mathrm{~cm}^{-1}$ at $700 \mathrm{~nm}$, and $\mu_{\mathrm{s}}$ is $83-250 \mathrm{~cm}^{-1}$ at 500

$\mathrm{nm}, 52-228 \mathrm{~cm}^{-1}$ at $600 \mathrm{~nm}$ and $35-211 \mathrm{~cm}^{-1}$ at $700 \mathrm{~nm}$.

Figure 4. 14 Graphic illustrations of (A) total diffuse reflectance measured from an arbitrary point on a tissue surface illuminated by a broad uniform beam and $(\mathrm{B})$ the fraction $\mathrm{f}$ associated with the collection geometry.

Figure 5. 1 Schematics of the non-contact RTD spectroscopy system.............................51

Figure 5. 2 Algorithm to estimate hemodynamic and structural characteristics..............55

Figure 5. 3 Validation of the proposed RTD spectral interpretation algorithm using Monte Carlo simulation.

Figure 5. 4 Results from the forepaw stimulation in old rats $(\mathrm{N}=2)$. The time histories of the indicative parameters were obtained from nine events induced by 5 -second stimulation, 18 events by 16 -second stimulation, and 8 events by 30 -second stimulation. The solid lines are the averaged time histories. The error bars represent the standard deviations. The gray dash-line marked the baseline of each indicative parameter.

Figure 5. 5 Results from the forepaw stimulation in younger rats $(\mathrm{N}=2)$. The time histories of the indicative parameters were obtained from 13 events induced by 5 -second stimulation, 12 events by 16 -second stimulation and 15 events by 30 -second stimulation. The solid lines are the averaged time histories. The error bars represent the standard deviations. The gray dash-line marked the baseline of each indicative parameter.

Figure 5. 6 Baseline $\mathrm{SatO}_{2}$ from 4 old rats and 4 young rats

Figure 6. 1 Flow chart of the image analysis procedures (Method 1). PCA:

principal component analysis.

Figure 6. 2 An example of images acquired by dynamic intrinsic optical imaging (DIOI) system (A) and commercial dSLR camera (B). An image was also taken with dSLR camera after the placement of electrocorticography (ECoG) electrode array (C), for the purpose of post-analysis comparison between DIOI and ECoG analyses.

Figure 6. 3 Analysis of dynamic intrinsic optical imaging (DIOI) data (Method 1). (A) and (B) were the raw images obtained by DIOI at $500 \mathrm{~nm}$ and $700 \mathrm{~nm}$, respectively. Spectral density maps (SDMs) at $500 \mathrm{~nm}(\mathrm{C})$ and $700 \mathrm{~nm}$ (D) show the power of hemodynamic low-frequency oscillations (LFOs). Correlation coefficient map (CCM) in (E) was obtained by calculating the correlation coefficient between LFOs at both wavelengths. K-means 
segmentation (F) was performed to isolate those regions with negative correlation $(\mathrm{G})$. These regions were later classified into multiple clusters $(\mathrm{H})$ by mean shift clustering. Each cluster consists of LFOs with unique temporal profiles (I). Granger causality toolbox was used to identify the Grangercause (J) and Granger-effect (M) in all these clusters. Seed-based CCM at both wavelengths were generated with regard to the Granger-cause ( $\mathrm{K}$ for $500 \mathrm{~nm}$, L for $700 \mathrm{~nm}$ ) and the Granger-effect ( $\mathrm{N}$ for $500 \mathrm{~nm}, \mathrm{O}$ for $700 \mathrm{~nm}$ ). Among all twelve cases with the resection areas inside the optical field of view, $83 \%$ of them showed the Granger-causes in epileptic cortex $(\mathrm{P})$.

Figure 6. 4 Differentiation between epileptogenic and eloquent cortex (Method 2). (A) Autoregressive model with exogenous source (ARX) at Order 11 shows the highest accuracy (0.84) and largest area under receiver operating characteristic (ROC) curve (0.82). (B) The corresponding impulse response functions (IRFs) obtained from epileptogenic (blue) and eloquent (red) areas. (C) The hyperplane obtained by support vector machine (SVM) could separate the epileptogenic cortex from eloquent areas with a sensitivity of $93 \%$ and a specificity of $70 \%(n=25,15$ epileptogenic and 10 eloquent areas). $\mathrm{HWD}=$ Haar wavelet decomposition.

Figure 6. 5 Vasculature abnormalities in the superficial layer (Method 3). A) Examples of vessel networks in epileptogenic and normal cortices and their corresponding histogram (blue: epileptogenic cortex, red: normal cortex). Areas showing normal cortex were randomly selected, regardless of their functional roles. B) Normalized histogram of the vessel network image obtained from the epileptogenic and normal cortices. Six patients (Patient 1, $2,5,7,8$, and 9) with high-quality color image of the exposed brain (Number of epileptogenic areas $=18$, number of normal areas $=20$ ). For each patient, the window sizes of the epileptogenic area and normal area were the same. The histograms for each patient were normalized to the highest value in epileptogenic areas' histograms. 


\section{Background}

Pediatric brain surgery is a major branch of the pediatric neurosurgery. It accounts for the diagnosis, evaluation, and treatment of neurological diseases and/or disorders. Two of the most common neurological diseases/disorders diagnosed in children are brain tumors and epilepsy. For those patients, surgical resection of brain lesions remains one of the optimal treatment options when applicable.

\subsection{Pediatric brain tumors}

A tumor is a mass of tissue formed by accumulated abnormal cells. In the brain, the tumor emerges from various cell types that make up the brain and central nervous system (CNS), such as astrocytes. Common pathophysiological traits of brain tumors include hypoxia and elevated hemoglobin concentration $[1,2]$. Brain tumors could be either benign (non-cancer) or malignant (cancer). When a brain tumor becomes sufficiently large, it may press the surrounding normal brain tissues and hence interrupt or stop their associated functions, and even cause seizures. Brain tumors are the most common solid tumor among children and are the leading cause of death in children [3, 4]. Unfortunately, the cause of most pediatric brain tumors is mostly unknown. The symptoms and signs of the pediatric brain tumors vary significantly from patient to patient, in terms of the location, size, growth speed, and the age of incidence.

The Central Brain Tumor Registry of the United States (CBTRUS) recently released their seventeenth statistical report [5] regarding the most up-to-date population-based summary of the current descriptive epidemiology of primary brain and central nervous system (CNS) tumors in the United States. The CBTRUS report was created based on the 
data collected from 51 population-based cancer registries, including 50 state registries and the District of Columbia, from 2007 to 2011.

According to this report, brain and CNS tumors are the most common neoplasm for children and adolescents between 0 and 19 years old, with an average annual ageadjusted incidence rate of 5.42 per 100,000 for a total count of 22,535 incident tumors. It was estimated that 4,620 new cases of primary malignant and benign brain and CNS tumors would be diagnosed among children and adolescents in the United States in 2015. Among 343,175 incident brain tumors reported during 2007-2011, 66\% of them $(227,376)$ were benign. Children and adolescents $(0-19$ years old) have the highest fiveyear relative survival rates $(73.3 \%)$ among all age groups diagnosed with primary malignant brain and CNS tumors. Five-year relative survival rate after diagnosis with a benign brain/CNS tumor is $91.9 \%$ in the United States. The three most common pediatric brain tumors are pilocytic astrocytomas, embryonal tumors, and glioma malignant, not otherwise specified (NOS) which account for $15.4 \%, 12.0 \%$, and $11.7 \%$, respectively. Gliomas account for approximately $47.9 \%$ of tumors in children and adolescents ages 0 19 years.

Surgery is the initial treatment for most benign and many malignant tumors following the diagnosis. It is often the most preferred treatment when a tumor can be removed without any unnecessary risk of neurological deficits. The surgery has two main goals. The first one, which is also the most important one, is to obtain a tissue biopsy to guide further management, such as the need of chemotherapy. The second goal is to remove all tumorous tissue if possible, while preserving the eloquent cortex as much as possible to 
reduce the post-surgical morbidity. There is a strong correlation between the extent of tumor removal and clinical outcomes (i.e., 10-year progression-free survival rate) [4, 610]. To achieve these two goals, accurate intraoperative delineation of brain tumors is desired.

\subsection{Pediatric epilepsy}

Epilepsy is a common and a complex neurological disorder that can affect health and quality of life in many ways. In epilepsy, groups of neurons in the brain sometimes signal abnormally and therefore, cause recurrent seizures. Epileptic seizures are especially detrimental to children because they may severely interrupt the development of normal brain functions. According to the Epilepsy Foundation, there are approximately 326,000 American children under the age of 15 who have epilepsy. And nearly 150,000 new cases

of epilepsy are diagnosed each year [11]. The incidence of epilepsy among children is much higher than that in other age groups.

There are many different ways to successfully control epileptic seizures [11]. The most common one is to treat the epilepsy with anti-seizure drugs. However, it could cause side effects to the patients and its effectiveness could diminish over time. Dietary approaches, such as high-fat, very low carbohydrate ketogenic diet, have often been used as the alternatives to treat medication-resistant epilepsy, but they are not easy to maintain as a result of their strict adherence to a limited range of foods. For patients with refractory epilepsy that cannot be controlled by either medication or dietary approaches, surgical removal of the seizure onset zone, if possible, is the optimal solution to control 
the recurrent seizures. In this case, a clear demarcation of the brain region responsible for seizure generation is crucial to the success of the epilepsy surgery.

\subsection{Intraoperative guidance for pediatric brain surgery}

Several techniques have been developed over the years to demarcate neoplastic and epileptogenic lesions intraoperatively. These technologies utilize either the structural or the functional characteristics to differentiate the brain lesions from the normal brain. Here, a brief review of the current intraoperative technologies for pediatric brain surgery guidance is presented as follows.

\subsubsection{Frameless stereotaxic neuronavigation}

Frameless stereotaxic neuronavigation is widely available as the intraoperative guidance tool for neurosurgeries [12]. It utilizes fiducial markers or optical sensors to determine the position of the surgical instruments with regard to the area of interest defined by the pre-operative anatomical and functional imaging study. However, as a result of the loss of cerebrospinal fluid and its mechanical integrity, the brain shifts and deforms significantly during the course of the craniotomy [12-14], which could compromise the accuracy of the neuronavigation solely based on pre-operative imaging data. Therefore, additional intraoperative imaging techniques would have to be employed for the purpose of correcting target localization errors induced by brain shift/deformation.

\subsubsection{Intraoperative magnetic resonance imaging}

Magnetic resonance imaging (MRI) has very high spatial resolution and offers excellent soft tissue contrast. Intraoperative MRI (iMRI) and intraoperative functional MRI (ifMRI) became a desirable technique to provide anatomical and functional 
mapping ever since its first implementation in a surgical imaging suite [15]. It has been reported that with iMRI/ifMRI, a maximum extent of resection, despite the lesion's proximity to eloquent brain cortex and fiber tracts, can be achieved, which leads to a favorable surgical outcome and acceptable neurological deficit rates [16].

However, iMRI and ifMRI require extremely high infrastructure standards and maintenance; only a handful of hospitals/research institutes in the world have the financial and technical means to provide these techniques in routine patient care. In addition, ifMRI requires a sophisticated control on the anesthetic level of the patient and an external stimulation to trigger the response in the brain. In addition, the functional mapping of fMRI relies on the selections of hemodynamic response function (HRF). With different HRFs, the locations of corresponding event-related functional areas may vary. Cannestra et al. found a spatiotemporal difference in localizations of sensorimotor cortex in human between using fMRI with different HRFs and using evoked potential mapping [17].

\subsubsection{Ultrasound}

Ultrasound imaging is a cost-effective intraoperative imaging modality that often is used as an alternative to iMRI in neuronavigation [18]. It is capable of detecting neoplastic tumors because of their hyperechoic characteristic, which is associated with the structural abnormalities of brain tumors $[19,20]$. While it is very accurate in detecting confined, deeply located remnants of low- or high-grade tumors, the accuracy of ultrasound imaging is limited when detecting superficial remnants [20]. In addition, 
ultrasound is less likely to differentiate tumor from peritumoral edema that is also hyperechoic [21, 22].

\subsubsection{Electrocorticography}

Electrocorticography $(\mathrm{ECoG})$ could be used both intraoperatively and chronically. It has been demonstrated as a valuable tool to identify eloquent cortical areas in awake patients [12, 23-25]. It could also be used to localize epileptic lesions: seizure foci may be identified during seizure activity, between seizures, or both [26]. In a clinical setting, ECoG is considered gold standard in demarcating the seizure onset zone.

However, the ECoG study is usually time-consuming. It also requires a complex anesthesiological regimen when used intraoperatively [12]. When ECoG is used in a chronic recording, an additional operative session will be required for electrode implementation, which is associated with an elevated risk of having hemorrhage, infection, or cerebral edema [26]. Additionally, its validity is mainly limited to the superficial cortical areas [12].

\subsubsection{Diffuse reflectance spectroscopy}

Optical spectroscopy uses light absorption and scattering to quantify tissue biochemical composition and morphological characteristics. It has the advantage of providing non-intrusive, automated tissue characterization in real-time, without removing tissue. To date, optical spectroscopy has been widely used to study pathological and physiological features at the tissue and cell levels in vivo and in vitro [27-32]. Diffuse reflectance spectroscopy (DRS) is one of the optical spectroscopy techniques commonly used in optical tissue characterization. It allows detection of tissue structure and 
biochemical composition through assessing the optical properties (i.e., absorption and reduced scattering coefficients) of tissue [33]. One common utility of DRS is the detection of tissue hemodynamics, based on the fact that oxy- and deoxy-hemoglobin $(\mathrm{Hb})$ possess unique absorption features [34]. Understanding and assessing $\mathrm{Hb}$ oxygenation and concentration provides valuable insights into the condition of tissues. It is not surprising, then, that regional $\mathrm{Hb}$ oxygenation and $\mathrm{Hb}$ concentration are highly sought information in tissue injury and disease diagnosis [35-40]. Optical diagnostic technologies provide a potential complimentary solution for intraoperative brain tumor demarcation, as demonstrated by several research groups [41-45]. However, its penetration depth is limited to superficial layer. The accuracy of the measurement could also be affected by excessive pressure applied on the brain tissue due to the hand movements [46-50].

\subsubsection{Dynamic intrinsic optical imaging}

Dynamic intrinsic optical imaging (DIOI) has been considered to hold extensive scientific potential that could provide more detailed understandings of the cortical microenvironment [13]. It usually involves imaging the in vivo brain at multiple wavelengths simultaneously, from which the blood volume and oxygenation information of the cortical surface can be retrieved [51]. DIOI has been evaluated for functional mapping in humans with electrocortical simulation [52] or peripheral stimulation [53, 54].

It has been more than two decades since Haglund et al. [55] observed significant changes in optical signals from the human cortical surface during seizure and cognitive tasks. However, DIOI was only used for research purposes, extensively on preclinical 
models of acute seizure [56-58] and chronic epilepsy [59] with regard to the neurovascular coupling, and has not yet been approved for application in clinical decisionmaking.

Current studies involved with animal models of seizure solely investigated how DIOI would reflect the seizure onset and propagation under very fine control of the location and timing of seizure induction [56-58]. As for the mapping of epileptogenic cortex in humans, the only available method relies on the onset of ictal episodes [55], which is hardly to achieve in an intraoperative setting. Nevertheless, little work has been attributed to investigate the feasibility of delineating the epileptogenic cortex during interictal periods. 


\section{Significance and goals}

An objective, real-time system that is capable of intraoperatively detecting the margins of pediatric brain tumors and epileptic cortex with high sensitivity would greatly aid neurosurgeons in their objective to safely and completely resect abnormal brain tissue without removing normal one. Intraoperative optical diagnostic techniques, such as diffuse reflectance spectroscopy (DRS) and dynamic intrinsic optical imaging (DIOI), are able to provide anatomical and functional mapping at relatively lower cost, without the concerns about the brain shifting and deformation after surgical procedures.

The primary goal of this $\mathrm{PhD}$ research is to demonstrate the feasibility of using various optical techniques in conjunction with sophisticated signal processing algorithms to differentiate brain tumors and epileptogenic cortex from normal brain tissue intraoperatively. Considering that neoplastic and epileptogenic brain lesions have their own unique pathophysiologic characteristics, optical techniques derived to differentiate them from the normal brain should be efficient in capturing the unique attributes. For example, brain tumors are usually characterized by high cell density, enlarged nuclei, and unique vessel network, hence, its static hemodynamic and structural properties should be the targeted biomarkers, based on which the optical tumor detection system should be developed. On the other hand, interictal epileptiform discharges could be frequently detected from the epileptic brain, which have been used to localize irritative zones

clinically. Therefore, DIOI might be able to capture those hemodynamic variations caused by interictal activities from the seizure onset zone within the exposed cortical surface during the surgery. 
These surgical guidance tools will ultimately differentiate brain tumors and epileptogenic cortex from normal brain based upon distinct intrinsic morphological, biochemical, and physiological attributes. The success of this $\mathrm{PhD}$ research should positively impact the management of pediatric brain tumors and epilepsy, because it will produce a new means by which neurosurgeons can objectively optimize the outcomes of brain tumor and epilepsy surgeries, thereby improving the prognoses of patients, and reducing the emotional and financial burdens endured by patients and their families. Moreover, the same system can be used for other applications, such as intraoperative monitoring of oxygen saturation level during bypass surgery.

Three major aims listed below describe the procedures of accomplishing the goal of this $\mathrm{PhD}$ research.

Aim 1: To develop a novel tissue differentiation algorithm for in vivo brain tumor detection using a probe-based diffuse reflectance spectroscopy system, and to compare its accuracy with histological results.

Aim 2: To devise a novel non-contact point spectroscopic detection system for in vivo brain tumor detection, and to derive a novel spectral interpretation algorithm to estimate useful hemodynamic and structural characteristics for in vivo tissue differentiation purposes.

Aim 3: To develop novel methodologies for intraoperative mapping of epileptogenic and eloquent cortex using a dynamic intrinsic optical imaging system. 


\section{Intraoperative pediatric brain tumor detection using diffuse reflectance spectroscopy}

\subsection{Introduction}

According to the statistical report based upon data collected from 2007 to 2011 across 50 states and the District of Columbia issued by the United States Central Brain Tumor Registry, brain tumors are the leading cause of death among all forms of pediatric cancer [5]. The five-year survival rates of some tumors, such as glioblastoma, are lower than $20 \%$ [5]. Among all the available treatment options, surgical removal is largely considered the primary and most desired course of action in most cases [60]. To achieve accurate demarcation of the brain tumor intraoperatively, various imaging modalities like magnetic resonance imaging (MRI), ultrasound, and optical techniques - have been utilized by different research groups to provide delineation of the margins of a brain tumor, and sometimes to assess the volume of residual tumor. Among these techniques, intraoperative MRI (iMRI) is considered to be the most advanced modality, because of its superior imaging resolution [61]. However, iMRI requires very high standard infrastructure, which is expensive both in installment and maintenance, and its image acquisition time is relatively long. A popular alternative is ultrasound, which is also capable of real-time imaging of brain tumors [19, 20]. However, its reliability in

detecting tumor is less than that of iMRI; Gerganov et al. compared the ultrasound technique with iMRI and pointed out that ultrasound could be used to detect more confined deeply located remnants of low- or high-grade tumors with high accuracy, though its accuracy is limited when detecting superficial remnants [20]. 
Optical diagnostic technologies provide another complimentary solution for intraoperative brain tumor demarcation, as they feature real-time feedback with functional information and high spatial resolution. Several studies have demonstrated the feasibility of using these technologies for differentiating brain tumors from normal brain, in vitro as well as in vivo [62-66]. Among the available optical diagnostic technologies, diffuse reflectance spectroscopy is the simplest and most affordable, in terms of implementation. It uses the principle of photon-tissue interaction to gauge the optical properties of biological tissue and, hence, indirectly assess its structural and physiological characteristics. Since hemoglobin is the dominant absorber of many in vivo tissues in the visible wavelength region, diffuse reflectance spectroscopy is often used to estimate the hemodynamic information of biological tissue $[67,68]$. We have previously demonstrated its potential to differentiate pediatric brain tumors from normal brain tissue, by simply analyzing the intensities of diffuse reflectance spectra in the near infrared region [64]. This chapter describes and examines a further improved tumor classification system with enhanced accuracy, which is achieved by the addition of functional parameters (i.e., hemoglobin concentration ([Hb]) and hemoglobin oxygen saturation level $\left.\left(\mathrm{SatO}_{2}\right)\right)$ and structural information (intensity of diffuse reflectance signal at 700 $\mathrm{nm}$, denoted as $\mathrm{Rd} 700$ ) estimated using diffuse reflectance signals in the visible wavelength region.

\subsection{Methods}

\subsubsection{Optical Instrumentation}

A tungsten halogen light source (LS-1, Ocean Optics, Florida), with an average output power of $6.5 \mathrm{~W}$, was used to illuminate the tissue. The intensity of diffused 
reflected photons was recorded by a spectrometer (USB 2000, Ocean Optics, Florida) with a spectral range of $240 \mathrm{~nm}$ to $932 \mathrm{~nm}$. Light delivery and collection were accomplished with a custom-made fiber-optic probe. The probe consisted of seven identical optical fibers with a $300-\mu \mathrm{m}$ core diameter and a 0.22 numerical aperture. One fiber was used to deliver illumination from the light source to the tissue surface; one was unused; and the other five were used to collect photons reflected from the tissue surface to the spectrometer. The average separation distance between the illumination fiber and collection fibers was roughly $300 \mu \mathrm{m}$. Further details on the fiber-optic system could be found in previous publications [63-65].

\subsubsection{Clinical data acquisition}

Twenty pediatric patients, between one and 18 years old, participated in this clinical study at Nicklaus Children's Hospital since 2007. The protocol of the clinical study was approved by the Western Institutional Review Board and the Institutional Review Board at Florida International University. Diffuse reflectance spectra were obtained from the in vivo brains of participants during craniotomy procedures for tumor resection. During spectral data acquisition, the optical probe was held by the neurosurgeons and was in direct contact with the brain tissue. Optical measurements were taken both from areas away from the resection zone (i.e., normal sites) and from areas within the resection zone (tumor sites). A set of diffuse reflectance spectra $(\mathrm{n}=5)$ was acquired from each investigation site with an integration time of 30-100 milliseconds. Following each spectral acquisition sequence, a baseline measurement was obtained by turning off the

illumination light source. At least two normal sites were investigated during each study 
for the first ten patients enrolled in a pilot study dated back to 2007-2008. For the other ten patients enrolled most recently since 2013, the control diffuse reflectance spectra were acquired from at least five normal sites. The number of investigated tumor sites was determined by the neurosurgeons based on the size and location of the brain tumor within the exposed cortical surface.

\subsubsection{Histology}

A specimen was collected by the neurosurgeons from each investigated site within the resection zone for histopathological evaluation to identify the type of brain tumor. The neurosurgeons were not aware of the analytical results of diffuse reflectance spectroscopy during the resection procedure. Biopsy samples were immediately fixed in a $5 \%$ formalin solution after resection. The specimens then were prepared for sectioning and hematoxylin and eosin staining. Processed slides were reviewed by a neuropathologist (Dr. Mahlon Johnson at University of Rochester) who was blinded to the study results and other clinical information. If any specimen had been identified as normal, the corresponding measured diffuse reflectance spectra were included in the normal groups in the following analyses. A summary of the numbers of investigated normal sites and tumor sites were provided in Table 3.1.

\subsubsection{Spectral data processing}

The baseline measurement $\operatorname{Rdbase}(\lambda)$ from each investigated site was first subtracted from the corresponding diffuse reflectance spectral set $\operatorname{Rd}(\lambda)$ to remove unwanted ambient light influences. Then, instrumentally-induced spectral alterations were eliminated by dividing the spectra by a calibration spectrum $\operatorname{Rd} d_{c a l}(\lambda)$. Note that the 
calibration spectrum was measured from a diffuse reflectance standard (FGS-20-02c, Avian Technologies, NH) using the same spectroscopic system. Mathematically speaking, the entire spectral calibration process can be described using the following equation: $\left[\operatorname{Rd}(\lambda)-\operatorname{Rd} \operatorname{base}_{\text {be }}(\lambda)\right] / \operatorname{Rd} d_{\text {cal }}(\lambda)$.

Three indicative parameters, extracted from each calibrated diffuse reflectance spectra, were used to characterize the site of investigation. These indicative parameters were $[\mathrm{Hb}], \mathrm{SatO}_{2}$, and $\mathrm{Rd} 700$. The methods for estimating $[\mathrm{Hb}]$ and $\mathrm{SatO}_{2}$ using diffuse reflectance spectra can be found in our previously published papers $[67,68]$. In order to reduce inevitable biological variations among all studied subjects, the indicative parameters of all measurements (both normal sites and tumor sites) from each patient were centered to the mean value and scaled based on the standard deviation of the indicative parameters from the normal sites of the same patient, which yielded normalized $\mathrm{n}[\mathrm{Hb}], \mathrm{nRd} 700$, and $\mathrm{nSatO}_{2}$. In addition, the distributions of $\mathrm{n}[\mathrm{Hb}], \mathrm{nRd} 700$, and $\mathrm{nSatO}_{2}$ from all normal sites and tumor sites of the studied patients were investigated.

\subsubsection{Tissue differentiation algorithm}

The normalized indicative parameters described in the previous section were used to establish a classification system to differentiate brain tumors from normal brain cortex. Support vector machine (SVM), a machine learning method, was employed in this study. Each indicative parameter was used as a stand-alone feature in the SVM to differentiate brain tumors from normal brain tissue. In addition, combinations of any two indicative parameters or all three of them were also used as classification features in SVM. The 
performance of SVM with different classification features (individual or combinations) was evaluated in terms of estimation accuracy, sensitivity and specificity,

$$
\left\{\begin{array}{l}
\text { Accuracy }=\frac{T P+T N}{P+N} \\
\text { Sensitivity }=\frac{T P}{T P+F N} \\
\text { Specificity }=\frac{T N}{T N+F P}
\end{array}\right.
$$

where TP is true positive, TN is true negative, FN is false negative, FP is false positive, $\mathrm{P}$ is positive (tumor), and $\mathrm{N}$ is negative (normal brain tissue).

\subsection{Results}

Demographic data on the patients and the tumor types identified by histology are listed in Table 3.1. The average age was $7.3 \pm 5.0$ years. A total of 82 control measurements were taken from the normal cerebral cortex and 53 from brain tumors.

Table 3. 1 Demographic data on the twenty patients who participated in the clinical study

\begin{tabular}{llllcc}
\hline $\begin{array}{l}\text { Case } \\
\#\end{array}$ & Gender & Age & Tumor type & $\begin{array}{c}\text { Number of } \\
\text { normal sites }\end{array}$ & $\begin{array}{c}\text { Number of } \\
\text { tumor sites }\end{array}$ \\
\hline 1 & M & 1 & Primitive neuroectodermal tumor & 2 & 2 \\
2 & F & 9 & Primitive neuroectodermal tumor & 2 & 1 \\
3 & M & 10 & Pilocytic astrocytoma & 2 & 2 \\
4 & F & 5 & Angioglioma & 2 & 3 \\
5 & F & 6 & Astrocytoma & 2 & 2 \\
6 & F & 9 & Astrocytoma & 2 & 1 \\
7 & F & 8 & Medulloblastoma & 2 & 3 \\
8 & F & 6 & Pilocytic astrocytoma & 2 & 2 \\
9 & F & 3 & Ganglioglioma & 2 & 5 \\
10 & F & 14 & Astrocytoma & 2 & 1
\end{tabular}




\begin{tabular}{|c|c|c|c|c|c|}
\hline 11 & M & 2 & Glioma & 6 & 8 \\
\hline 12 & M & 5 & Medulloblastoma & 6 & 4 \\
\hline 13 & $\mathrm{~F}$ & 16 & Glioblastoma & 5 & 3 \\
\hline 14 & M & 7 & Ganglioglioma & 5 & 1 \\
\hline 15 & $\mathrm{~F}$ & 3 & $\begin{array}{l}\text { Dysembryoplastic neuroepithelial } \\
\text { tumor }\end{array}$ & 6 & 3 \\
\hline 16 & M & 8 & Atypical teratoid rhabdoid tumor & 9 & 1 \\
\hline 17 & $\mathrm{~F}$ & 13 & Craniopharyngioma & 8 & 1 \\
\hline 18 & M & 1 & Anaplastic Astrocytoma & 5 & 6 \\
\hline 19 & M & 2 & $\begin{array}{l}\text { Embryonal tumor with abundant } \\
\text { neuropil and true rosettes }\end{array}$ & 5 & 2 \\
\hline 20 & M & 18 & Brainstem Glioma & 7 & 2 \\
\hline
\end{tabular}

(a)

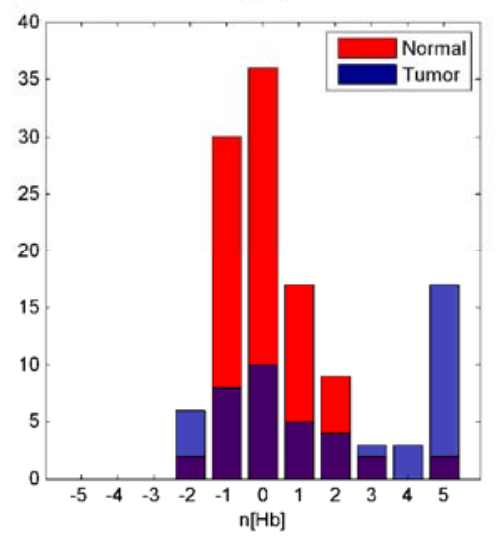

(b)

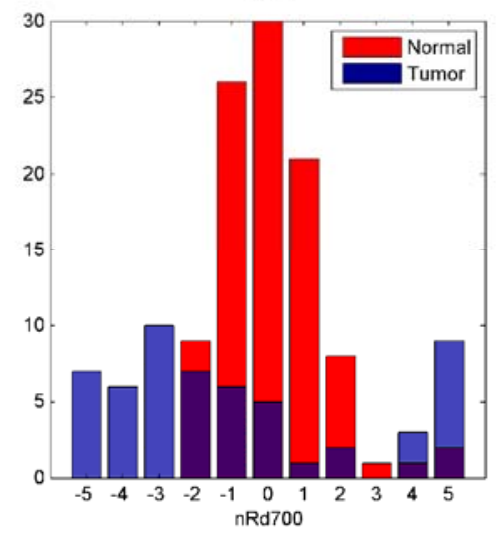

(c)

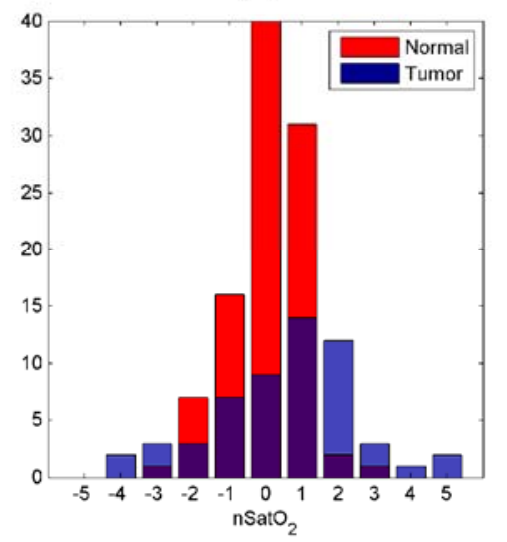

Figure 3. 1 Histograms of (a) $n[\mathrm{Hb}]$, (b) $n R d 700$ and (c) $n \mathrm{SatO}_{2}$ from normal and neoplastic brain tissue.

We noticed that raw $[\mathrm{Hb}], \mathrm{Rd} 700$ and $\mathrm{SatO}_{2}$ from normal cortex are patientdependent. Using the patient-dependent normalization approach, we found that the distribution of normalized indicative parameters (i.e., $\mathrm{n}[\mathrm{Hb}], \mathrm{nRd} 700$ and $\mathrm{nSatO}_{2}$ ) from normal cortex became more concentrated, exhibiting standard normal distribution characteristics (Fig. 3.1 in red). In contrast, the distribution of normalized indicative parameters from tumor tissue was not following a standard normal distribution (Fig. 3.1 
in blue), where both $\mathrm{n}[\mathrm{Hb}]$ and $\mathrm{nRd} 700$ tend to distribute much more widely than those from normal cortex (Fig. 3.1a and 3.1b). The distribution of $\mathrm{nSatO}_{2}$ in tumor tissue was slightly left-skewed (Fig. 3.1c in blue). All these results indicate that tumors generally contained a higher $[\mathrm{Hb}]$ and more irregular structure than normal cortex. In general, the average of $\mathrm{nRd} 700$ from tumor measurements was lower than that from normal brain, although some tumor sites may show much higher $\mathrm{nRd} 700$ as a result of the proximity of white matter. The $\mathrm{nSatO}_{2}$ of the tissue under investigation might not be sufficient in itself to discriminate the tumor from the normal tissue, since it yielded the worst performance when applied in SVM to differentiate the tumor from normal tissue as shown in Table 3.2 .

(a)

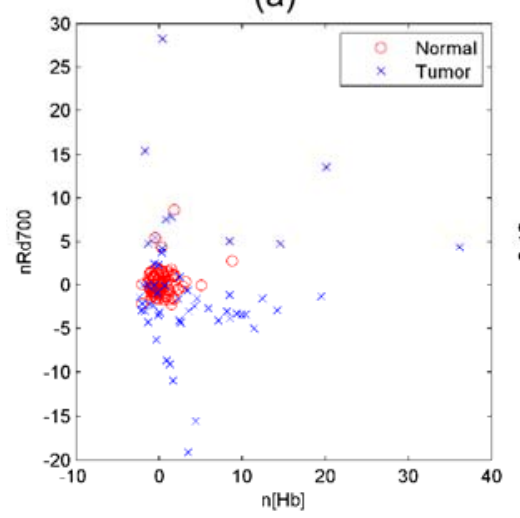

(b)

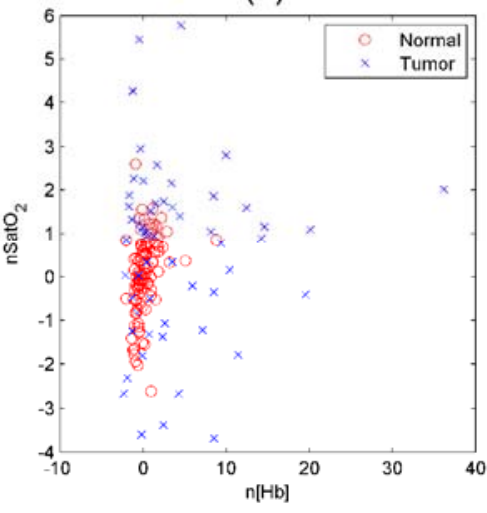

(c)

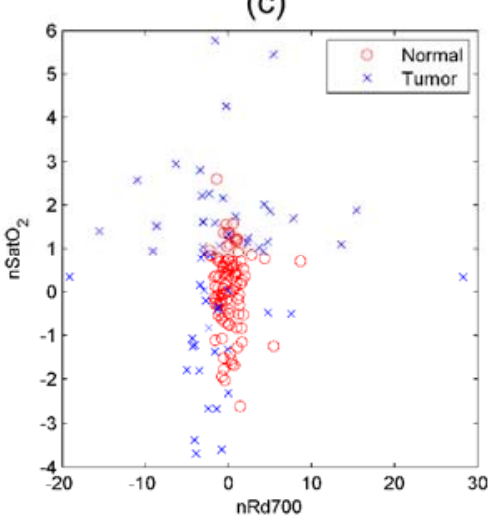

Figure 3. 2 Scatter plot of (a) $n[\mathrm{Hb}]$ and $n R d 700$, (b) $n[\mathrm{Hb}]$ and $n \mathrm{SatO}_{2}$, and (c) $n R d 700$ and $n \mathrm{SatO}_{2}$ from normal cortex and brain tumor.

Fig. 3.2 depicts the distribution of measurements from normal and tumor sites in terms of pairs of normalized indicative parameters. It became more obvious that the normalized indicative parameters from tumor sites are more scattered comparing to those obtained from normal sites. The performance of the tissue differentiation algorithm with 
SVM (Table 3.2) was also improved when pairing up these normalized indicative parameters.

When all three normalized indicative parameters from normal and tumor sites were plotted in a three-dimensional Cartesian coordinate system (Fig. 3.3), the indicative parameters from normal cortex were clearly confined within a cluster centered at zero, which could be wrapped around within a hyperplane defined by SVM; in contrast, parameters from tumors tended to scatter outside the normal hyperplane. The performance of the tissue differentiation with SVM when using all three normalized indicative parameters simultaneously was the best one among all, in terms of accuracy (92\%), sensitivity (91\%) and specificity (93\%) shown in Table 3.2 .

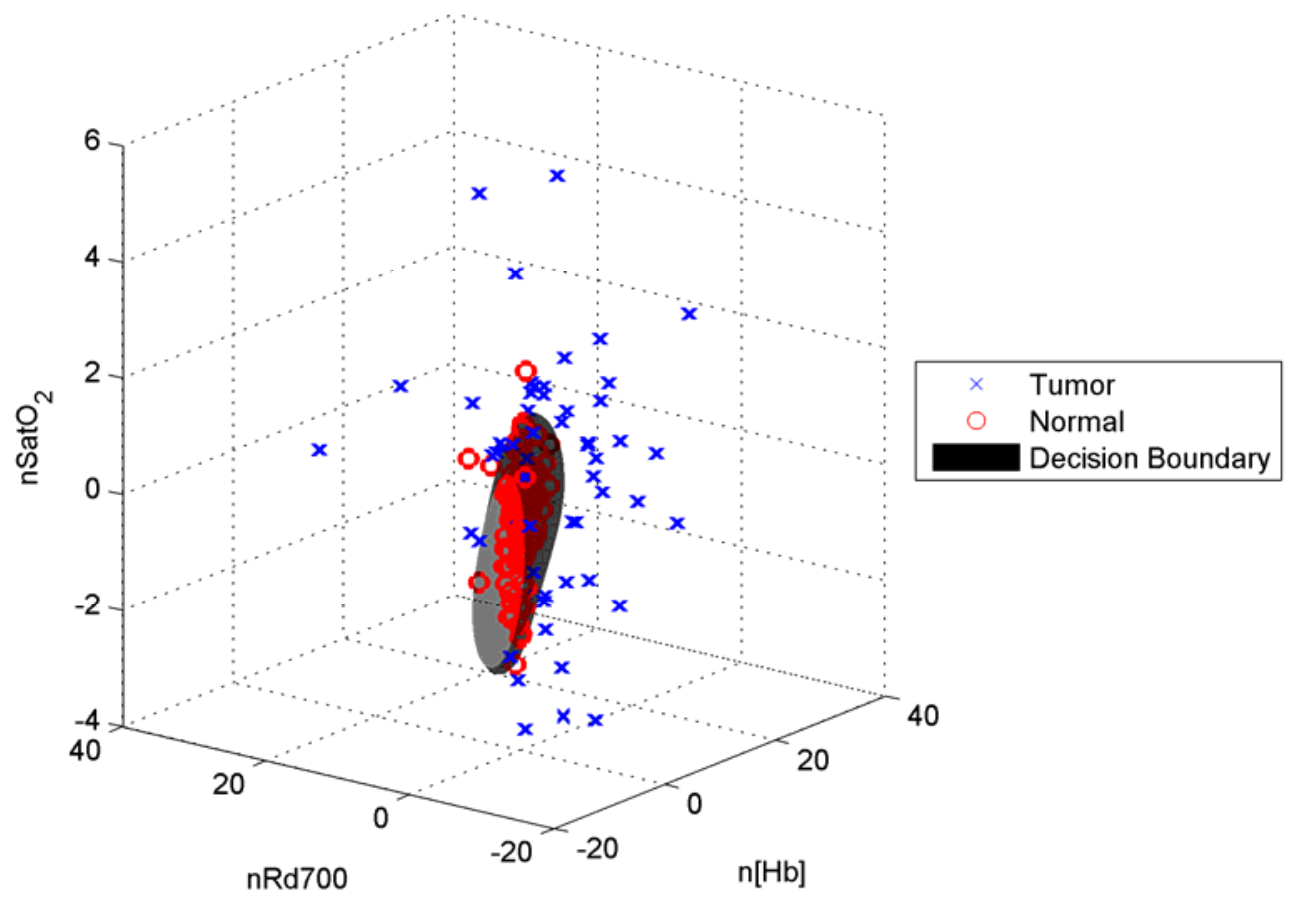

Figure 3. 3 The distribution of tumor and normal tissue sites, in accordance with their corresponding $n[H b], n R d 700$, andnSatO ${ }_{2}$. The black hyperplane that differentiate brain tumors from normal cortex was obtained from support vector 
machine based on all three normalized indicative parameters. The performance of the differentiation is shown in Table 3.2 .

Table 3. 2 Tissue differentiation accuracy, sensitivity, and specificity for three individual classification criteria and four combined classification criterion with support vector machine.

\begin{tabular}{cccc}
\hline Parameter & Accuracy & Sensitivity & Specificity \\
\hline $\mathrm{n}[\mathrm{Hb}]$ & 0.78 & 0.50 & 0.94 \\
$\mathrm{nRd700}$ & 0.84 & 0.70 & 0.93 \\
$\mathrm{nSatO} 2$ & 0.72 & 0.61 & 0.79 \\
$\mathrm{n}[\mathrm{Hb}]$ and $\mathrm{nRd} 700$ & 0.88 & 0.77 & 0.94 \\
$\mathrm{n}[\mathrm{Hb}]$ and $\mathrm{nSatO}_{2}$ & 0.84 & 0.75 & 0.89 \\
$\mathrm{nRd700}$ and $\mathrm{nSatO}_{2}$ & 0.88 & 0.84 & 0.91 \\
$\mathrm{n}[\mathrm{Hb}], \mathrm{nRd} 700$ and $\mathrm{nSatO}_{2}$ & 0.92 & 0.91 & 0.93 \\
\hline
\end{tabular}

\subsection{Discussion}

In this study, we tested the feasibility of using diffuse reflectance spectroscopy to differentiate pediatric brain tumor from normal brain tissue intraoperatively. Our results suggest that three indicative parameters derived from in vivo diffuse reflectance spectra [Hb], Rd700 and $\mathrm{SatO}_{2}$ - clearly differentiate brain tumor tissue from normal cortex. Although these indicative parameters each individually differentiated the tumor specimens from normal cortex with moderate accuracy, the highest discrimination accuracy (92\%) was generated using an algorithm based on support vector machine incorporating all three parameters. This observation again confirms the feasibility of developing an intelligent guidance system for pediatric brain tumor surgery using diffuse reflectance spectroscopy.

As in a previous study [64], we found that diffuse reflectance signals in the longer wavelength region (i.e., above $650 \mathrm{~nm}$ ), where blood absorption is minimal, are very 
effective at differentiating brain tumors from normal cortex. Diffuse reflectance signals in this wavelength region usually are strongly influenced by the scattering properties of the biological tissue, which are linked to the tissue's structural characteristics [69]. While alterations in the morphological characteristics of brain tumors are readily confirmed by histological studies of pediatric brain tumors, their associations with low diffuse reflectance signals (i.e., low scattering properties) are not yet clearly understood. We note that the low scattering characteristic of pediatric brain tumors is unique, given that adult brain tumors usually exhibit a greater degree of scattering than normal cortex [70, 71]. This discrepancy, in our opinion, should be attributed to structural/compositional differences between adult and pediatric brain tumors [64]. In this study, too few measurements from normal white matter were collected to include them in analysis. Nevertheless, we are confident that diffuse reflectance signals in longer wavelength regions will be highly efficient at differentiating white matter from pediatric brain tumors. This conjecture is supported by several previously-published studies that have demonstrated that diffuse reflectance signals from white matter are notably stronger than those from cerebral cortex, due to the former's high scattering characteristics [70-72].

Direct use of diffuse reflectance signals between 400 and $600 \mathrm{~nm}$ could not produce effective algorithms differentiating brain tumor from normal cortex. Using the spectral interpretation algorithms developed by our group, we converted the signal in this region to the absolute quantities of $[\mathrm{Hb}]$ and $\mathrm{SatO}_{2}$. As shown in this study, the addition of this hemodynamic information enhances the efficiency of the discrimination algorithm for pediatric brain tumors. This success may be explained by the unique hemodynamic characteristics of brain tumors: they tend to be hypoxic and possess high hemoglobin 
concentrations $[1,2]$. From the data reported here, we also note great variations in tumor $\mathrm{SatO}_{2}$ and $[\mathrm{Hb}]$. We attribute these data characteristics to the fact that brain tumor morphology is highly inhomogeneous, mostly consisting of a necrotic core and angiogenic rim $[73,74]$. Therefore, the location of measurements could dictate the signals obtained. In addition to enhancing tumor demarcation, understanding the in vivo physiological characteristics of brain tumors may help to predict the effectiveness of adjuvant chemotherapy and radiation therapy [75-77]. This, in turn, may influence the aggressiveness of surgical resection. It should be noted that this functional information is not available in today's intraoperative environment.

The methodology utilized for in vivo diffuse reflectance spectral acquisition in this study may produce some unwanted effects on the in vivo hemodynamic information of the investigated site. One concern relates to the employment of a contact probe, since probe contact pressure can influence local $\mathrm{SatO}_{2}$ and $[\mathrm{Hb}]$ considerably [46]. During a single data acquisition procedure, which is approximately 1-2 seconds in duration, the probe is held by the neurosurgeon's hand and thereby subject to movement. This phenomenon is evidenced by the large temporal variations in $\mathrm{SatO}_{2}$ and $[\mathrm{Hb}]$ we observed within some recordings. To avoid this, it will be necessary to reduce the time of investigation and to maintain steady probe contact pressure, which will highly rely on the neurosurgeons' experience. Alternatively, our group is developing a new data acquisition scheme which does not require a contact probe, a scheme we intend to test in a future study. 
A second issue of concern is that high $[\mathrm{Hb}]$ readings from brain tumor may be misleading, because, during surgery, bleeding at the resection site is inevitable, and surface blood contamination may increase $[\mathrm{Hb}]$ readings. Therefore, cautions should be made to irrigate the cortical surface and clean the remaining blood contamination prior to each measurement.

\subsection{Conclusions}

In this study, diffuse reflectance spectroscopy was applied to determine the structural and hemodynamic characteristics of in vivo brain tissue during the pediatric brain tumor surgery. With the help of SVM, these intrinsic properties can be used to efficiently differentiate pediatric brain tumor from normal cortex with very high accuracy in an intraoperative environment. The encouraging results of this study support the concept of using diffuse reflectance spectroscopy to develop an intraoperative surgical guidance system for pediatric brain tumor surgery. 


\section{Determination of the optical properties of turbid media using total diffuse reflectance}

\subsection{Introduction}

Optical properties of biological tissues are always of great interest to researchers in the field of biomedical optics because of their intimate relationships with the intrinsic structural and compositional tissue characteristics. This is especially true for applications in investigating tissue pathology, tissue injury, and tissue functionality. Many techniques have been developed to measure optical properties from in vivo biological tissues over the past two decades [78-84]. Among them, spatially and temporally resolved diffuse reflectance signals are the most commonly used; they can be conveniently acquired using a contact fiber optic bundle [78-80, 83]. However, a significant drawback is associated with the utility of a contact fiber optic probe: the contact pressure introduced by the probe significantly alters the measured optical properties in vivo [46, 49]. One possible approach to eliminate this drawback is to acquire desired optical signals in a non-contact fashion. Over the past decade, several non-contact techniques for in vivo optical property measurements have been proposed and developed: Cuccia et al. introduced a non-contact, wide-field measurement technique of optical properties using spatially modulated illumination and achieved excellent accuracy [85]; Bish et al. used a lens system to image the illumination and collection fibers onto the tissue surface and hence achieve noncontact measurements of radially dependent diffuse reflectance [86]; Foschum et al. introduced two apparatus to carry out non-contact measurements of spatially resolved reflectance and total reflectance from that absorption and reduced scattering coefficients individually estimated [87]. However, these techniques require either a sophisticated 
optical system to, for example, specially modulate the illumination or a carefully controlled collection geometry, which make them less suitable for intraoperative applications such as tumor resection guidance.

In this study, a new hybrid spectroscopy imaging system was devised for the purpose of acquiring relative total diffuse reflectance spectra from a given point within the field of view of the system [88]. In addition, the system is capable of obtaining two-dimensional relative total diffuse reflectance signals at a specific wavelength. By incorporating a reference measurement and a look-up table established using a Monte Carlo (MC) simulation model for photon migration, relative total diffuse reflectance signals were converted to absolute total diffuse reflectance signals and subsequently the ratios of the reduced scattering coefficient to the absorption coefficient (i.e., $\mu_{\mathrm{s}}{ }^{\prime} / \mu_{\mathrm{a}}$ ). The accuracy of the system in terms of measuring $\mu_{\mathrm{s}}{ }^{\prime} / \mu_{\mathrm{a}}$ was first demonstrated theoretically using total diffuse reflectance signals generated by the $\mathrm{MC}$ model, and then experimentally using optical phantoms.

\subsection{Materials and Methods}

\subsubsection{Instrumentation}

Design details for the hybrid spectroscopy imaging system are depicted in Fig. 4.1. The system consisted of two signal acquisition modalities - the imaging modality and the point spectroscopic detection modality. The targeting sample was imaged through either a Nikon dSLR lens (Nikon AF 28-80 mm f/3.5-5.6 D lens with aperture ring) or a zoom imaging lens (VZM 450i, Edmund Optics Inc., Barrington, NJ). The image from the camera lens was collimated using a Hastings triplet achromatic lens (\#30-229, EFL 40.3 mm, Edmund Optics Inc., Barrington, NJ) and transmitted to a beam splitter (\#54-824, 
50R/50T, Edmund Optics Inc., Barrington, NJ). The collimated image beam transmitting through the beam splitter would enter into the imaging modality of the system; it was further divided by a dichroic mirror with a transmission band of 400-595 nm and a reflection band of 640-750 nm (\#49-471, Edmund Optics Inc., Barrington, NJ). Two narrow band-pass filters with center passing wavelengths at $500 \mathrm{~nm}$ (\#65-149, Edmund Optics Inc., Barrington, NJ) and 700 nm (\#88-012, Edmund Optics Inc., Barrington, NJ) were placed at the transmission side and the reflection side of the dichroic mirror, respectively. The collimated image beam transmitting through the band-pass filter was refocused onto the sensor of a CCD camera (DMK 21AU04, The Imaging Source Europe $\mathrm{GmbH})$ using a Hastings lens. The collimated image beam reflected by the beam splitter would enter into the point spectroscopic detection modality and be re-focused using a Hastings lens. At the image plane of this lens, an optical fiber (core diameter $50 \mu \mathrm{m}$, $\mathrm{NA}=0.2$, GIF50C, Thorlabs Inc., Newton, NJ) was used to collect light from the center of the formed image. The distal end of the optical fiber was connected to either a red laser diode module (CPS184, $650 \mathrm{~nm}, 4.5 \mathrm{~mW}$, Thorlabs Inc., Newton, NJ) assembled inhouse to track the location of investigation on the targeting sample, or a spectrometer (USB2000, Ocean Optics, Dunedin, FL) to record light reflected by the sample. The area of investigation of the point spectroscopic detection modality was determined by the magnification of the lens as well as the diameter of the optical fiber. The hybrid system was operated through a couple of LabVIEW programs developed in-house. The illumination of the sample investigated was provided by an external light source. Assuming the illumination is relatively uniform, the light received by a pixel of the camera or the point spectroscopic detection system should be considered as a fraction of 
the total diffuse reflectance $\left(\mathrm{R}_{\mathrm{TD}}\right)$ generated by a pencil beam illumination, in accordance with the principle of convolution [89]. The proof for this concept is provided in the $\mathbf{4 . 6}$

\section{Appendix.}

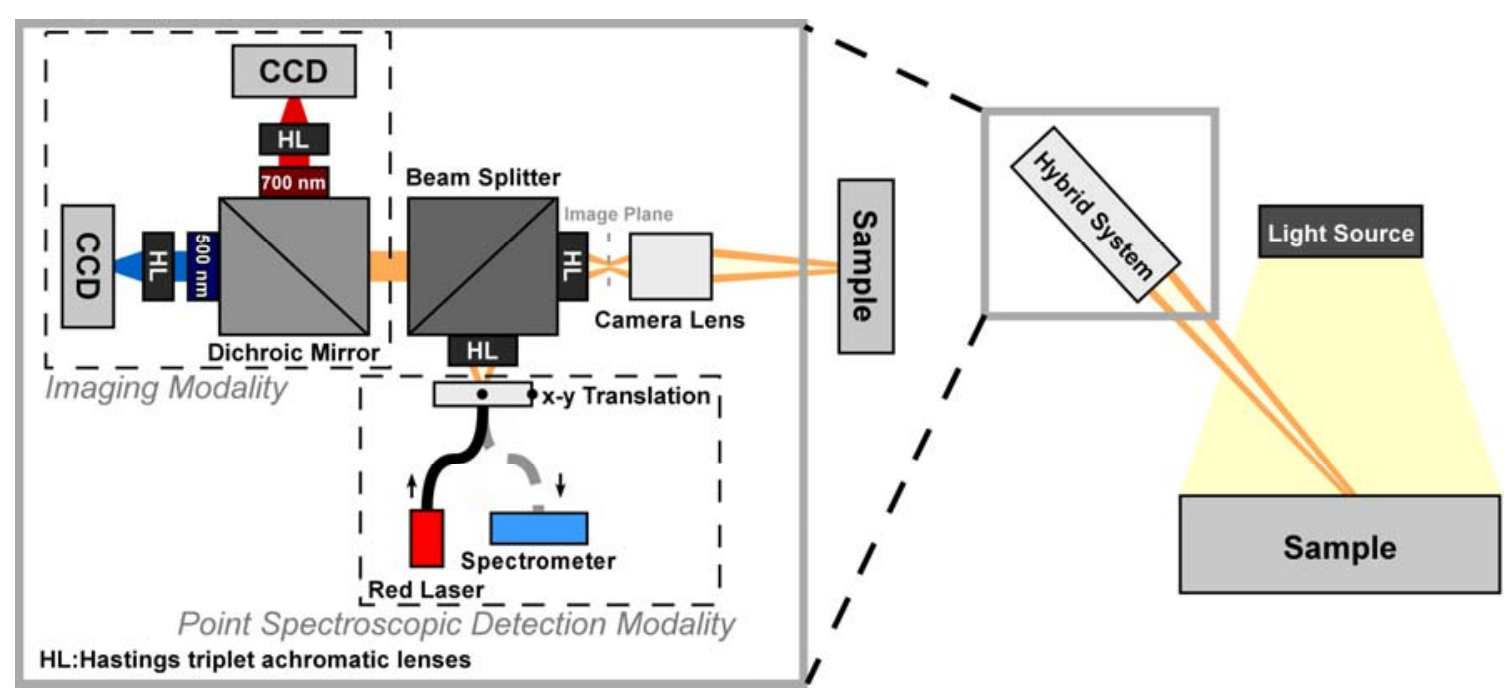

Figure 4. 1 The schematic of the hybrid spectroscopy imaging system.

\subsubsection{Look-up table for $\mu_{\mathrm{s}}{ }^{\prime} / \mu_{\mathrm{a}}$ estimation based on total diffuse reflectance $\left(\mathbf{R}_{\mathrm{TD}}\right)$}

According to the theory proposed by Farrell et al., $\mathrm{R}_{\mathrm{TD}}$ is only a function of $\mu_{\mathrm{s}}{ }^{\prime} / \mu_{\mathrm{a}}[81]$. To easily convert RTD measured by the hybrid system to $\mu_{\mathrm{s}}{ }^{\prime} / \mu_{\mathrm{a}}$, a look-up table was constructed using a $\mathrm{MC}$ simulation model for photon migration in tissue in a slab geometry. The simulation model was developed and verified using MCML provided by Wang et al. [90]. The magnitudes of $\mu_{\mathrm{s}}{ }^{\prime} / \mu_{\mathrm{a}}$ used to create the look-up table are depicted in Table 4.1, with a randomly selected fixed value of $\mu_{\mathrm{a}}\left(2 \mathrm{~cm}^{-1}\right)$. In the simulations, the tissue slab was illuminated by a pencil beam at a normal angle. The refractive index $\mathbf{n}$, the anisotropy factor $\mathbf{g}$, and the thickness of the simulated tissue slab were 1.395, 0.88, and $100 \mathrm{~cm}$, respectively. The total number of photons used in each simulation was 10 million. The resulting look-up table is shown in Fig. 4.2. 
Table 4. 1 Ranges of $\mu_{s}{ }^{\prime} / \mu_{a}$ used in look-up table building

\begin{tabular}{|l|c|c|c|}
\hline \multicolumn{5}{|c|}{$\mu_{\mathrm{s}}{ }^{\prime} / \mu_{\mathrm{a}}$} \\
\hline & Start Value & Increment & End Value \\
\hline Range 1 & 0 & 0.01 & 0.99 \\
\hline Range 2 & 1 & 1 & 100 \\
\hline Range 3 & 110 & 10 & 200 \\
\hline Range 4 & 250 & 50 & 500 \\
\hline Range 5 & 600 & 100 & 1000 \\
\hline Range 6 & 2000 & 1000 & 10000 \\
\hline
\end{tabular}

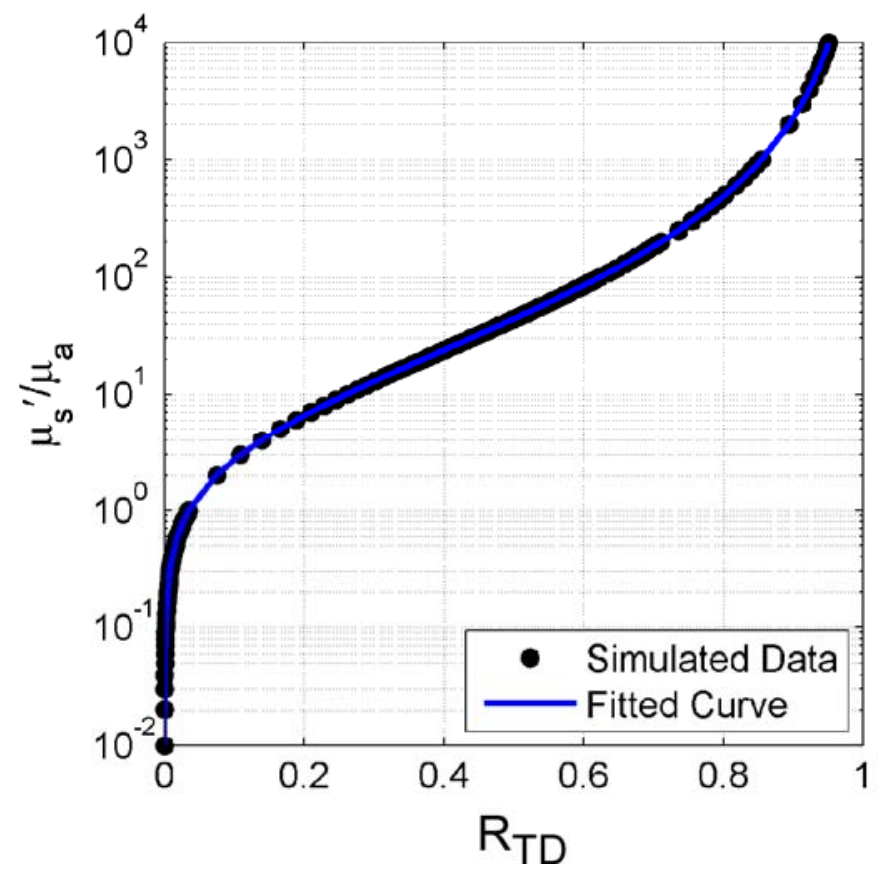

Figure 4. 2 Relationships between total diffuse reflectance $R_{T D}$ and $\mu_{s}^{\prime} / \mu_{a}$. Black dots are $R_{T D}$ derived from the Monte Carlo simulations. The blue line is the fitted curve obtained using the Curve Fitting Toolbox of Matlab.

\subsubsection{Evaluation of the accuracy of the look-up table using Monte Carlo simulation}




\subsubsection{Constant $\boldsymbol{n}$ and $\boldsymbol{g}$}

A Matlab program was developed to generate 50 random $\mu_{\mathrm{s}}-\mu_{\mathrm{a}}$ pairs for each of the six $\mu_{\mathrm{s}}{ }^{\prime} / \mu_{\mathrm{a}}$ ranges depicted in Table 4.1. These coefficients, in conjunction with $\mathbf{n}=1.395$ and $\mathbf{g}=0.88$, were applied to the $\mathrm{MC}$ simulation model to generate $\mathrm{R}_{\mathrm{TD}}$, assuming the simulated tissue phantom is infinitely thick and illuminated by a pencil beam at a normal angle. Each simulated RTD was converted to $\mu_{\mathrm{s}}{ }^{\prime} / \mu_{\mathrm{a}}$ using the look-up table of $\mu_{\mathrm{s}}{ }^{\prime} / \mu_{\mathrm{a}}$ verse RTD shown in Fig. 4.2. Each estimated $\mu_{\mathrm{s}}{ }^{\prime} / \mu_{\mathrm{a}}$ value was subsequently compared with the corresponding input $\mu_{\mathrm{s}} / \mu_{\mathrm{a}}$ values to determine the absolute percentage error of estimation $|\Delta \mathrm{E}|$. That is

$$
|\Delta E|=\left|\frac{v_{2}-v_{1}}{v_{1}}\right| \times 100 \%,
$$

where $\mathrm{v}_{1}$ and $\mathrm{v}_{2}$ are the input and the estimated $\mu_{\mathrm{s}}{ }^{\prime} / \mu_{\mathrm{a}}$ values, respectively.

\subsubsection{Varying $\boldsymbol{g}$}

The changes in a look-up table of $\mu_{\mathrm{s}}{ }^{\prime} / \mu_{\mathrm{a}}$ verse RTD resulting from $\mathbf{g}$ variation were investigated in a quantitative fashion. The $\mu_{\mathrm{s}}{ }^{\prime}-\mu_{\mathrm{a}}$ pairs generated in the previous section were used in conjunction with $\mathbf{n}=1.4$ and $\mathbf{g}=0.85,0.90$ or 0.95 in the $\mathrm{MC}$ simulation model to simulate $\mathrm{R}_{\mathrm{TD}}$. A reference look-up table of $\mu_{\mathrm{s}}{ }^{\prime} / \mu_{\mathrm{a}}$ verse $\mathrm{R}_{\mathrm{TD}}$ was established using the simulation results with $\mathbf{g}=0.85$ and, subsequently, the simulated $\mathrm{R}_{\mathrm{TD}}$ with $\mathbf{g}=$ 0.90 and $\mathbf{g}=0.95$ were applied to this look-up table to retrieve the estimated $\mu_{\mathrm{s}}{ }^{\prime} / \mu_{\mathrm{a}}$ values. Finally, the discrepancies between the estimated and the input $\mu_{s}{ }^{\prime} / \mu_{a}$ were quantified using Eq. (4.1). Additionally, the input $\mu_{\mathrm{s}}{ }^{\prime} / \mu_{\mathrm{a}}$ values were applied to the look-up table of $\mu_{\mathrm{s}}{ }^{\prime} / \mu_{\mathrm{a}}$ verse $\mathrm{R}_{\mathrm{TD}}$ for $\mathbf{g}=0.85$ to estimate $\mathrm{R}_{\mathrm{TD}}$. The discrepancies between the estimated 
and the simulated $\mathrm{R}_{\mathrm{TD}}$ were again quantified using Eq. (4.1), with v1 being the simulated $\mathrm{R}_{\mathrm{TD}}$ and $\mathrm{v} 2$ the estimated one.

\subsubsection{Varying $n$}

To evaluate the effects of $\mathbf{n}$ on a look-up table of $\mu_{\mathrm{s}}{ }^{\prime} / \mu_{\mathrm{a}}$ verse $\mathrm{RTD}$, the input $\mu_{\mathrm{s}}{ }^{\prime} / \mu_{\mathrm{a}}$ values created in the previous section were used in conjunction with $\mathbf{g}=0.9$ and $\mathbf{n}=1.3$, 1.4 or 1.5 in a MC simulation model to simulate RTD. A reference look-up table of $\mu_{\mathrm{s}}{ }^{\prime} / \mu_{\mathrm{a}}$ verse $\mathrm{R}_{\mathrm{TD}}$ was established using the simulation results with $\mathbf{n}=1.3$ and, subsequently, the simulated $\mathrm{R}_{\text {TD }}$ with $\mathbf{n}=1.4$ and $\mathbf{n}=1.5$ were applied to this look-up table to retrieve the estimated values of $\mu_{\mathrm{s}} \mathrm{s} / \mu_{\mathrm{a}}$. Again, the discrepancies between the estimated and the input $\mu_{\mathrm{s}}{ }^{\prime} / \mu_{\mathrm{a}}$ were quantified using Eq. (4.1). Additionally, the input $\mu_{\mathrm{s}}{ }^{\prime} / \mu_{\mathrm{a}}$ values were applied to the look-up table of $\mu_{\mathrm{s}}{ }^{\prime} / \mu_{\mathrm{a}}$ verse $\mathrm{R}_{\mathrm{TD}}$ for $\mathbf{n}=1.3$ to estimate $\mathrm{R}_{\mathrm{TD}}$. The discrepancies between the estimated and the simulated $\mathrm{R}_{\mathrm{TD}}$ were again quantified using Eq. (4.1), with V1 being the simulated $\mathrm{R}_{T D}$ and $\mathrm{v} 2$ the estimated $\mathrm{RTD}$.

\subsubsection{Varying $\boldsymbol{g}$ and $\boldsymbol{n}$}

A similar Matlab program was developed to generate a set of 50 random $\mu_{\mathrm{s}}$ ' and $\mu_{\mathrm{a}}$ for each of the following conditions: 1) $\mathbf{n}=1.395$ and $\mathbf{g}$ varying between 0.85 and 0.95 ; 2) $\mathbf{n}$ varying between 1.3 and 1.5 and $\mathbf{g}=0.9$; and 3) $\mathbf{n}$ varying between 1.3 and 1.5 and $\mathbf{g}$ varying between 0.85 and 0.95 . Applying these optical property sets to the $\mathrm{MC}$ simulation model, simulated $\mathrm{R}_{\mathrm{TD}}$ were obtained and converted to $\mu_{\mathrm{s}}{ }^{\prime} / \mu_{\mathrm{a}}$ using the look-up table shown in Fig. 4.2. Each estimated $\mu_{\mathrm{s}}{ }^{\prime} / \mu_{\mathrm{a}}$ was compared with the corresponding input $\mu_{\mathrm{s}} / \mu_{\mathrm{a}}$ value to determine the absolute percentage error of estimation defined in Eq. 


\subsubsection{Evaluation of point spectroscopic detection modality of the hybrid system using optical phantoms}

The objective of this study was to investigate the feasibility and the accuracy of the point spectroscopic detection modality (see Fig. 4.1) of the hybrid system in measuring $\mu_{\mathrm{s}}{ }^{\prime} / \mu_{\mathrm{a}}$ from absorbing/scattering media. Nine cylindrical polyurethane optical phantoms with different optical properties were used in this study and were made in accordance with the recipe developed by Prahl et al. [91]. The optical properties (i.e., $\mu_{\mathrm{s}}$ and $\mu_{\mathrm{a}}$ ) of these phantoms within the visible wavelength range were determined using the double integrating sphere method [92], and their representative values are shown in Table 4.2. The refractive index of the cured polyurethane phantom is around 1.468 at $670 \mathrm{~nm}$ [93].

In order to measure absolute $\mathrm{R}_{\mathrm{TD}}$, a priori knowledge of the incident light intensity as well as the collection geometry of the hybrid system would be required, as suggested by Foschum et al. [87]. Unfortunately, these two pieces of information were difficult to obtain accurately. To circumvent this limitation, reference $\mathrm{R}_{\mathrm{TD}}$ measurements from a material with known optical properties was introduced to the process of obtaining absolute $\mathrm{R}_{\mathrm{TD}}$ and hence estimating $\mu_{\mathrm{s}} \mathrm{\prime} / \mu_{\mathrm{a}}$. Two types of materials were identified and employed as the reference materials here. Detailed measurement and data interpretation protocols of the evaluation study are provided as follows.

\subsubsection{Reference material as an optical phantom}

The setup and the measurement protocol of the sub-study are graphically depicted in Fig. 4.3. Each phantom was illuminated directly from above with a broad-band white light source (DC-950H, Dolan-Jenner, Edmund Optics Inc., Barrington, NJ); a fraction of RTD was measured through the point spectroscopic detection modality of the hybrid 
system at an arbitrary angle. The acquired signal was denoted as relative total diffuse reflectance spectrum $R_{x x x \_ \text {measure }}(\lambda)$, where $\mathrm{xxx}$ can be either 'ref', shortened for reference, or 'eva', shortened for evaluation. The side of each phantom used in this study was covered by a piece of black paper to ensure that only its top surface was illuminated during the study. In a single measurement procedure, the reference measurements were first carried out: a set of $10 R_{\text {ref_measure }}(\lambda)$ were acquired from a location close to the center of the top surface of the reference phantom. Without changing the illumination and collection geometries, the reference phantom was carefully replaced with an evaluation phantom. A set of 10 Reva_measure $(\lambda)$ were acquired from a location close to the center of the top surface of the evaluation phantom. The Reva_measure $(\lambda)$ acquisition procedure was repeated at five different locations. Prior to the acquisition of each set of relative total diffuse reflectance spectra, a dark spectrum $R_{\operatorname{dark}}(\lambda)$ was recorded without the illumination to access ambient light influence. Since the optical properties of the reference phantom were known, its theoretical absolute total diffuse reflectance spectrum $\mathrm{R}_{\mathrm{TD} \_ \text {ref }}(\lambda)$ could be estimated using a MC simulation. Subsequently, the absolute total diffuse reflectance spectrum from an evaluation phantom, $\mathrm{R}_{\mathrm{TD} \_ \text {eva }}(\lambda)$, could be calculated using:

$$
R_{T D_{\_} \text {eva }}(\lambda)=\frac{R_{\text {eva_measure }}(\lambda)-R_{\text {dark }}(\lambda)}{R_{\text {ref_measure }}(\lambda)-R_{\text {dark }}(\lambda)} \cdot R_{T D_{-} \text {ref }}(\lambda) .
$$

Two comparisons were made to access the accuracy of the spectroscopic pointdetection modality of the hybrid system in measuring $\mu_{\mathrm{s}}{ }^{\prime} / \mu_{\mathrm{a}}$ from absorbing/scattering media. Firstly, RTD_eva $(\lambda)$ was compared with theoretical total diffuse reflectance $\operatorname{RTD}_{-}$theory $(\lambda)$ derived from the look-up table (Fig. 4.2) using $\mu_{\mathrm{s}}{ }^{\prime}(\lambda) / \mu_{\mathrm{a}}(\lambda)$ of the evaluation 
phantom, predetermined by the double integrating sphere technique, as the input. Secondly, RTD_eva $(\lambda)$ was converted to $\mu_{\mathrm{s}}{ }^{\prime}(\lambda) / \mu_{\mathrm{a}}(\lambda)$ using the look-up table (Fig. 4.2) and then compared with $\mu_{\mathrm{s}}{ }^{\prime}(\lambda) / \mu_{\mathrm{a}}(\lambda)$ of the evaluation phantom predetermined by the double integrating sphere technique.

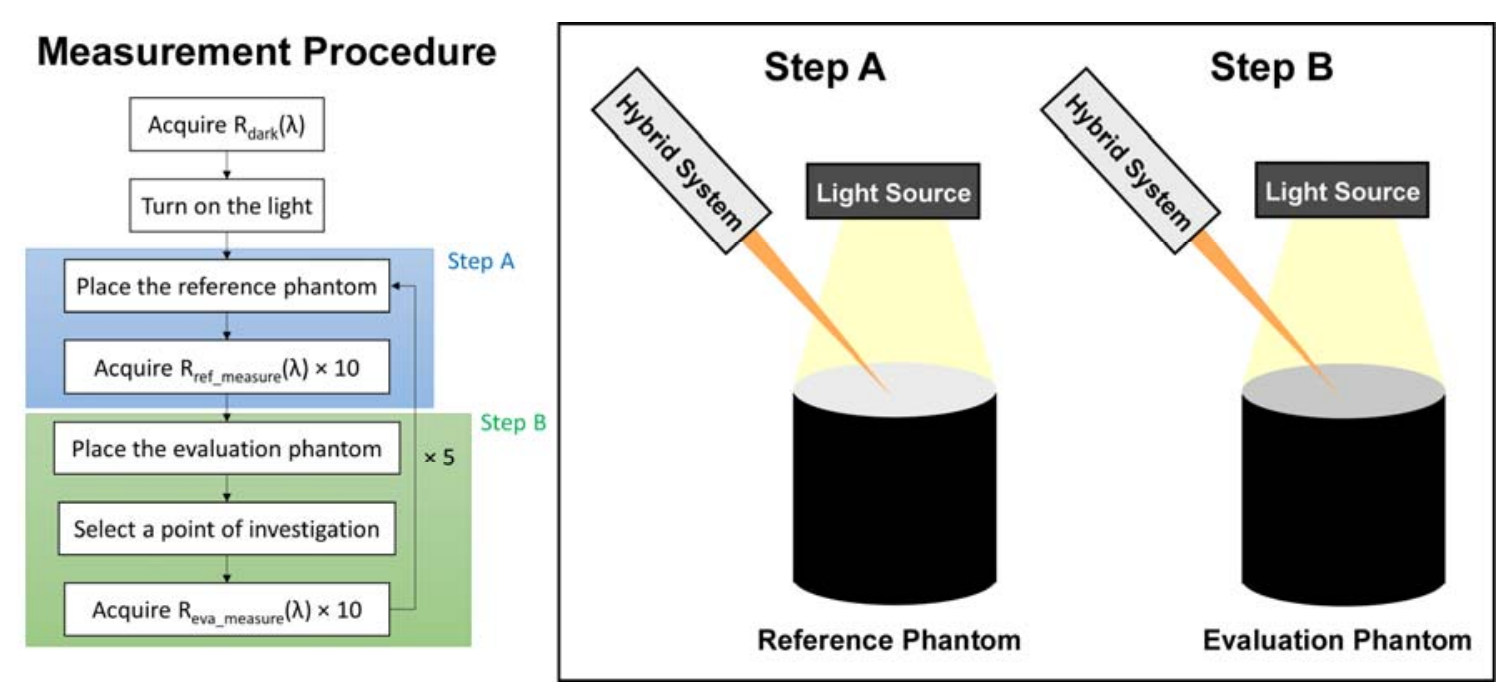

Figure 4. 3 Experimental setup for evaluating the function of the spectroscopic point-detection of the hybrid system. The flow chart on the left shows the measurement procedure.

\subsubsection{Reference material as a non-transparent, reflective paper}

The challenge of using an optical phantom as a reference lies in in vivo implementation: it would be difficult to maintain the same illumination and collection geometries between the phantom and the in vivo tissue of investigations. One way to address the in vivo applicability of a reference phantom is to create a reflective material that is thin and flexible enough to be placed directly on top of the in vivo tissue for reference measurements. A double-layer paper-based standard was therefore created and evaluated in this study. The top layer of this standard was a piece of reflective white paper; and the bottom layer a piece of black paper absorbing all light transmitting through the top layer. The experimental setup and the measurement procedure of this sub-study 
were similar to those depicted in Fig. 4.4. RTD_paper $(\lambda)$ was first determined using an optical phantom with known optical properties and Eq. (4.2). In the subsequent measurements, Rref_measure $(\lambda)$ was obtained from the paper-based standard placed on top of the evaluation phantom. Finally, RTD_eva $(\lambda)$ was calculated using:

$$
R_{T D_{-} \text {eva }}(\lambda)=\frac{R_{\text {eva_measure }}(\lambda)-R_{\text {dark }}(\lambda)}{R_{\text {paper_eva_measure }}(\lambda)-R_{\text {dark }}(\lambda)} \cdot \underbrace{\frac{R_{\text {paper_ref_measure }}(\lambda)-R_{\text {dark }}(\lambda)}{R_{\text {ref_measure }}(\lambda)-R_{\text {dark }}(\lambda)} \cdot R_{T D_{-} \text {ref }}(\lambda)}_{R_{T D \_p a v e r}(\lambda)} .
$$

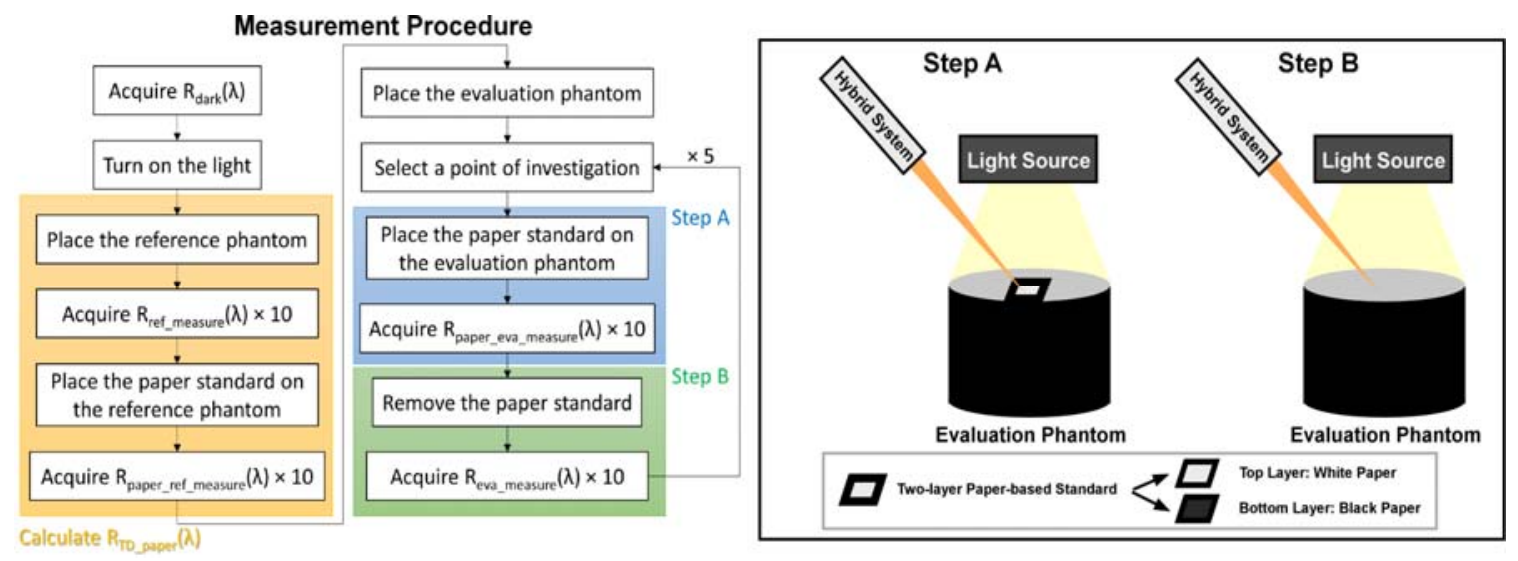

Figure 4. 4 Experimental setup for evaluating the function of the spectroscopic point-detection of the hybrid system. The flow chart on the top shows the measurement procedure.

To access the accuracy of the spectroscopic point-detection modality of the hybrid system in measuring $\mu_{\mathrm{s}}{ }^{\prime} / \mu_{\mathrm{a}}$ from absorbing/scattering media using a paper-based standard, two comparisons were made. Specifically, RTD_eva $(\lambda)$ was compared with theoretical total diffuse reflectance $\operatorname{RTD}_{-}$theory $(\lambda)$ derived from the look-up table (Fig. 4.2) using $\mu_{\mathrm{s}}{ }^{\prime}(\lambda) / \mu_{\mathrm{a}}(\lambda)$ of the evaluation phantom, predetermined by the double integrating sphere technique, as the input. In addition, $\mathrm{R}_{\mathrm{TD}}$ eva $(\lambda)$ was converted to $\mu_{\mathrm{s}}{ }^{\prime}(\lambda) / \mu_{\mathrm{a}}(\lambda)$ using the look-up table (Fig. 4.2) and then compared with $\mu_{\mathrm{s}}{ }^{\prime}(\lambda) / \mu_{\mathrm{a}}(\lambda)$ of the evaluation phantom predetermined by the double integrating sphere technique. 


\subsubsection{Evaluation of the imaging modality of the hybrid system using optical phantoms}

An evaluation experiment was also carried out with the imaging modality of the hybrid system to demonstrate its capability to measure $\mathrm{R}_{\mathrm{TD}}$ and hence estimate $\mu_{\mathrm{s}}{ }^{\prime} / \mu_{\mathrm{a}}$. The reference and evaluation phantoms were placed side-by-side under broad-area illumination. A large piece of thick reflective paper was first placed on top of both phantoms to capture the spatial profile of illumination $I_{p a p e r}(x, y)$ at $500 \mathrm{~nm}$ and $700 \mathrm{~nm}$. With the paper removed, the images of the top surfaces of the phantoms, Iphantom(x.y) were acquired at $500 \mathrm{~nm}$ and $700 \mathrm{~nm}$, at a rate of 7.5 frames per second for 30 seconds. To remove the artifacts originated from the spatial non-uniformity of illumination,

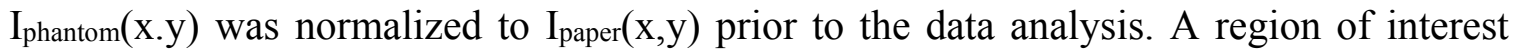
was randomly selected from the normalized image of each phantom, from which the mean intensity, either $\mathrm{R}_{\text {ref_measure }}$ or Reva_measure, was extracted. Finally, $\mathrm{R}_{\mathrm{TD} \_ \text {eva }}$ of each evaluation phantom was calculated using Eq. (4.2), and it was convert to $\mu_{\mathrm{s}}{ }^{\prime} / \mu_{\mathrm{a}}$ for accuracy assessment. Since the true optical properties of the phantoms at $700 \mathrm{~nm}$ were not available, the calculation results at $700 \mathrm{~nm}$ were not reported here.

\subsection{Results}

The effects of varying $\mathbf{g}$ on the relationship between $\mu_{\mathrm{s}}{ }^{\prime} / \mu_{\mathrm{a}}$ and $\mathrm{R}_{\mathrm{TD}}$ are summarized in Fig. 4.5. It is noticed that, within the range of investigation, increase in $\mathbf{g}$ variation does elevate the magnitude of $|\Delta \mathrm{E}|$. While the maximum $|\Delta \mathrm{E}|$ in $\mathrm{R}_{\mathrm{TD}}$ estimation using $\mu_{\mathrm{s}}{ }^{\prime} / \mu_{\mathrm{a}}$ could exceeds $10 \%$ when $\mu_{\mathrm{s}}{ }^{\prime} / \mu_{\mathrm{a}}$ smaller than 1 (Fig. $4.5 \mathrm{~B}$ ), $|\Delta \mathrm{E}|$ in $\mu_{\mathrm{s}}{ }^{\prime} / \mu_{\mathrm{a}}$ estimations based on RTD is reasonable small $(<2 \%)$ over the entire range of RTD (Fig. 
4.5A). This suggests that the look-up table for $\mu_{\mathrm{s}}{ }^{\prime} / \mu_{\mathrm{a}}$ verse $\mathrm{RTD}$ established with $\mathbf{g}=0.88$ (Fig. 4.2) is applicable to biological tissues with different $\mathbf{g}$ values.
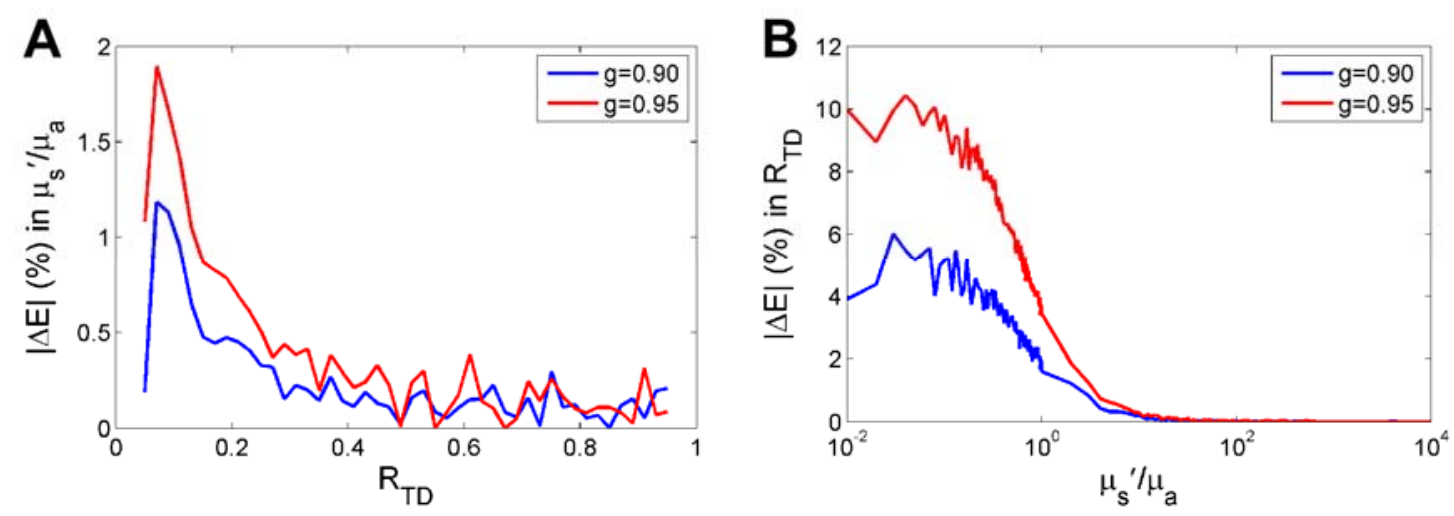

Figure 4. 5 A) Absolute percentage error $|\Delta E|$ in $\mu_{s} ' \mu_{a}$ estimations as a function of $R T D$ and anisotropy factor (g). B) Absolute percentage error $|\Delta E|$ in RTD estimations as a function of $\mu_{s}^{\prime} / \mu_{a}$ and $\boldsymbol{g}$. The look-up table of $\mu_{s}{ }^{\prime} / \mu_{a}$ verse $R T D$ with $\boldsymbol{g}=0.85$ was used for the conversion process, and $|\Delta E|$ was calculated using Eq. (4.1).

The effect of varying $\mathbf{n}$ on the relationship between $\mu_{\mathrm{s}}{ }^{\prime} / \mu_{\mathrm{a}}$ and $\mathrm{R}_{\mathrm{TD}}$ are summarized in Fig. 4.6. Similar to $\mathbf{g}$ variation, increase in $\mathbf{n}$ variation also raises the magnitude of $|\Delta E|$. In addition, $|\Delta \mathrm{E}|$ in $\mu_{\mathrm{s}}{ }^{\prime} / \mu_{\mathrm{a}}$ estimations using $\mathrm{R}_{\mathrm{TD}}$ steadily increases as $\mathrm{R}_{\mathrm{TD}}$ approaches to 1 . $|\Delta \mathrm{E}|$ in RTD estimations using $\mu_{\mathrm{s}}{ }^{\prime} / \mu_{\mathrm{a}}$, on the other hand, decreases as $\mu_{\mathrm{s}}{ }^{\prime} / \mu_{\mathrm{a}}$ increases. It is noticed that $|\Delta \mathrm{E}|$ induced by $\mathbf{n}$ variation is much greater than that induced by $\mathbf{g}$ variation; the maximum $|\Delta \mathrm{E}|$ exceeds $60 \%$ in $\mu_{\mathrm{s}}{ }^{\prime} / \mu_{\mathrm{a}}$ estimations using RTD (Fig. 4.6A) and $30 \%$ in RTD estimations using $\mu_{\mathrm{s}}{ }^{\prime} / \mu_{\mathrm{a}}$ (Fig. 4.6B). This clearly indicates the look-up table depicted in Fig. 4.2 is not applicable to biological tissues whose $\mathbf{n}$ greatly deviates (i.e, more than $5 \%)$ from 1.395. 

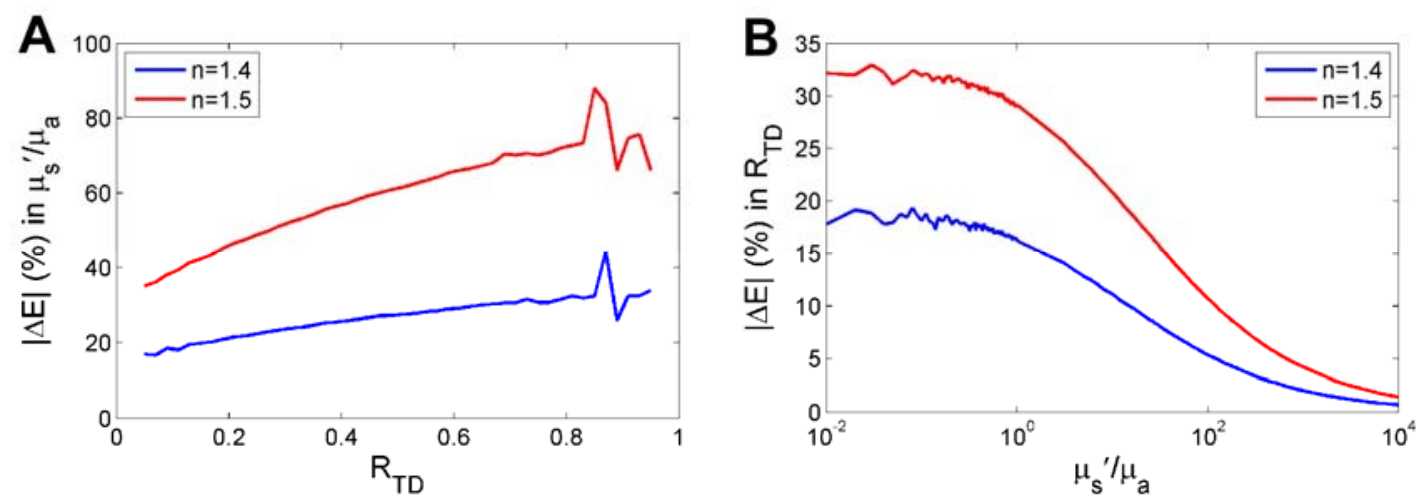

Figure 4. 6 A) Absolute percentage error $|\Delta E|$ in $\mu_{s}^{\prime} / \mu_{a}$ estimations as a function of $R_{T D}$ and refractive index (n). B) Absolute percentage error $|\Delta E|$ in $R_{T D}$ estimations as a function of $\mu_{s}^{\prime} ' / \mu_{a}$ and $\boldsymbol{n}$. The look-up table of $\mu_{s}^{\prime} / \mu_{a}$ verse $R_{T D}$ with $\boldsymbol{n}=1.3$ was used in al all conversion processes, and $|\Delta E|$ was calculated using Eq. (4.1).

The effects of varying $\mathbf{n}$ and $\mathbf{g}$ on $\mu_{\mathrm{s}} / \mu_{\mathrm{a}}$ estimations based on RTD are collectively summarized in Fig. 4.7. As expected, the accuracy of the $\mu_{\mathrm{s}}{ }^{\prime} / \mu_{\mathrm{a}}$ estimation is insensitive to $\mathbf{g}$ variation when $\mathbf{n}$ is a constant; the plot of input and the estimated $\mu_{\mathrm{s}}{ }^{\prime} / \mu_{\mathrm{a}}$ under such a condition shows a highly linear relationship $\left(\mathrm{R}^{2} \approx 1\right)$ with slope $=1$. However, the accuracy of the $\mu_{\mathrm{s}} / \mu_{\mathrm{a}}$ estimation suffers greatly when $\mathbf{n}$ varies.
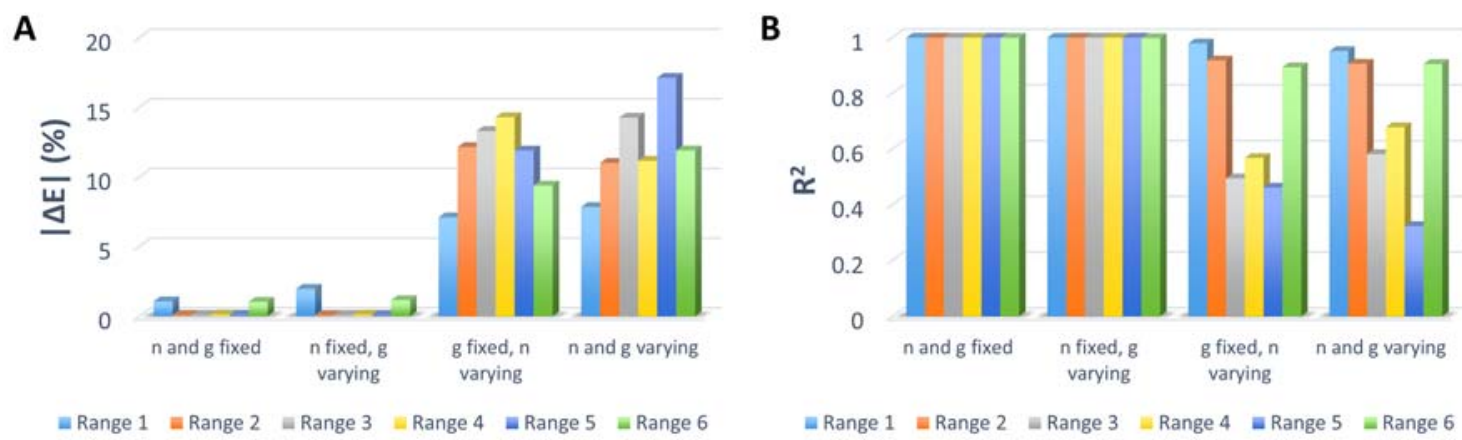

Figure 4. 7 A) Absolute percentage error $|\Delta E|$ in $\mu_{s}^{\prime} ' \mu_{a}$ estimations and B) correlation coefficients of the linear fitting between the input and the estimated $\mu_{s}^{\prime} / \mu_{a}$ values under four different conditions of $\boldsymbol{n}$ and $\boldsymbol{g}$, over the range of $\mu_{s} ' / \mu_{a}$ depicted in Table 4.1.

The results of experimental evaluation using optical phantoms demonstrate the high accuracy of the point spectroscopic detection modality of the hybrid system in terms of measuring $\mathrm{RTD}$ and estimating $\mu_{\mathrm{s}}{ }^{\prime} / \mu_{\mathrm{a}}$. The averaged $|\Delta \mathrm{E}|$ in $\mu_{\mathrm{s}}{ }^{\prime} / \mu_{\mathrm{a}}$ estimation within the wavelength range of 500-640 $\mathrm{nm}$ is approximately $9.34 \pm 6.86 \%$ (mean \pm standard 
deviation), as shown in Fig. 4.8. When $\mathrm{R}_{\text {TD_eva }}(\lambda)$ is plotted against $\mathrm{R}_{\mathrm{TD}}$ theory $(\lambda)$, a strong linear relationship with slope close to 1 is noticed for the entire wavelength range evaluated. A representative plot of such a relationship is provided in Fig. 4.9A, where $\mathrm{R}^{2}$ of linear regression is 0.986 . Furthermore, the same linear characteristic is noticed in the measured $\mu_{\mathrm{s}}{ }^{\prime}(\lambda) / \mu_{\mathrm{a}}(\lambda)$ verse theoretical $\mu_{\mathrm{s}}{ }^{\prime}(\lambda) / \mu_{\mathrm{a}}(\lambda)$ plots over the entire wavelength range evaluated. A representative plot of such a relationship is provided in Fig. 4.9B, where $\mathrm{R}^{2}$ of the linear regression is 0.935 . One interesting observation from this set of data is that the phantom itself is not a perfect homogenous medium. In several optical phantoms, a rather large variation is observed among Reva_measure $(\lambda)$ acquired from five random locations on the top surface of a given phantom. Therefore, only the mean of the five acquired Reva_measure $(\lambda)$ from a phantom is used to derive $\mathrm{R}_{\text {TD_eva }}(\lambda)$, which is then compared with $\mathrm{R}_{\mathrm{TD} \_ \text {theory }}(\lambda)$.

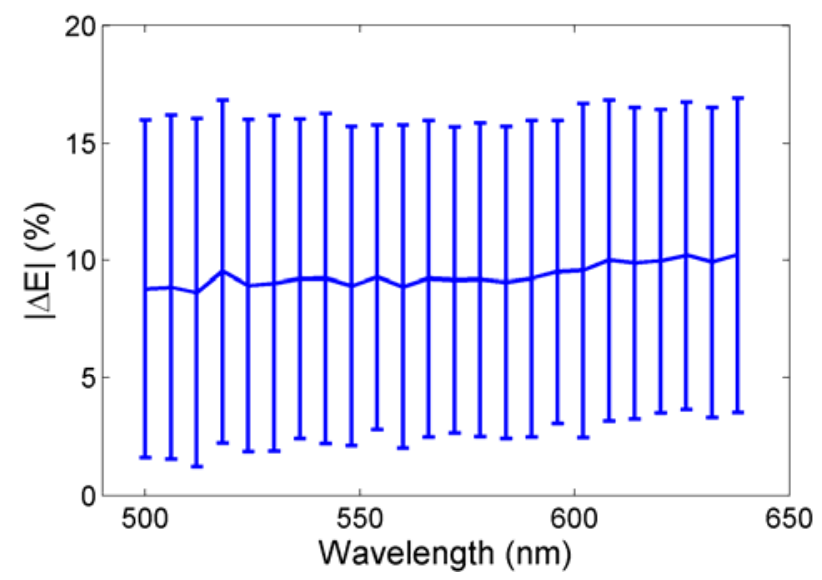

Figure 4. 8 Absolute percentage error $|\Delta E|$ in $\mu_{s}^{\prime}(\lambda) / \mu_{a}(\lambda)$ estimations using $R_{T D_{-} e v a}(\lambda)$ between $500 \mathrm{~nm}$ and $640 \mathrm{~nm}$. The references used are $\mu_{s}^{\prime}(\lambda) / \mu_{a}(\lambda)$ of the optical phantoms determined by the double integrating sphere technique. The middle line represents the mean $|\Delta E|$ calculated from all 8 phantoms; the error bars the standard deviation. One optical phantom was used as the reference standard in this study. 

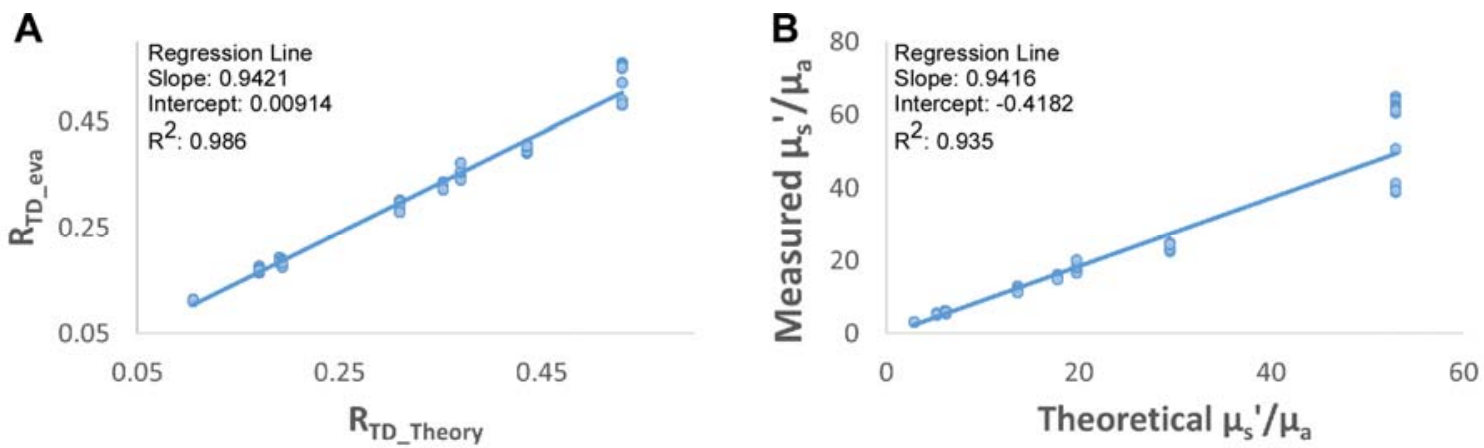

Figure 4. 9 Representative results from the experimental evaluation study of the point spectroscopic detection modality of the hybrid system. An optical phantom was used as the reference standard in this study. A) $R_{T D}$ eva $(\lambda)$ verse $R_{T D_{\text {_theory }}}(\lambda)$ at $\lambda=633 \mathrm{~nm}$. B) Measured $\mu_{s^{\prime}}{ }^{\prime}(\lambda) / \mu_{a}(\lambda)$ verse theoretical $\mu_{s}{ }^{\prime}(\lambda) / \mu_{a}(\lambda)$ at $\lambda=633 \mathrm{~nm}$. The solid lines in the figures are the linear regression lines.

When the reference optical phantom was replaced with a paper-based reference standard, the accuracy of the point spectroscopic detection modality of the hybrid system in terms of measuring $\mathrm{R}_{\mathrm{TD}}$ and estimating $\mu_{\mathrm{s}}{ }^{\prime} / \mu_{\mathrm{a}}$ remained and, in some cases, improved (Fig. 4.10). For example, the average $|\Delta \mathrm{E}|$ of $\mu_{\mathrm{s}} / \mu_{\mathrm{a}}$ estimation within the wavelength range of $500-640 \mathrm{~nm}$ is $5.54 \pm 4.6 \%$ (mean \pm standard deviation). In addition, a strong linear relationship is observed between $\mathrm{R}_{\mathrm{TD} \_ \text {eva }}(\lambda)$ and $\mathrm{R}_{\mathrm{TD} \text { _theory }}(\lambda)$ and between measured $\mu_{\mathrm{s}}{ }^{\prime}(\lambda) / \mu_{\mathrm{a}}(\lambda)$ and theoretical $\mu_{\mathrm{s}}{ }^{\prime}(\lambda) / \mu_{\mathrm{a}}(\lambda)$, as illustrated in Fig. 4.11. This improvement is attributed to the fact that consistent measurements can be obtained easily from the paperbased reference standard. 


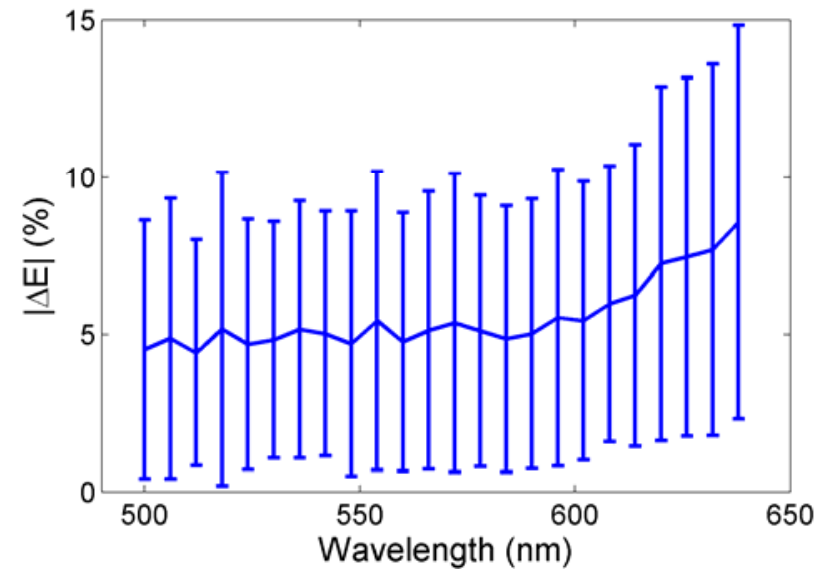

Figure 4. 10 Absolute percentage error $|\Delta E|$ of $\mu_{s}^{\prime}(\lambda) / \mu_{a}(\lambda)$ estimations between $500 \mathrm{~nm}$ and $640 \mathrm{~nm}$. The references used are $\mu_{s}^{\prime} / \mu_{a}$ of the optical phantoms determined by the double integrating sphere technique. The middle line represents the mean $|\Delta E|$ calculated from all 8 phantoms; the error bar the standard deviation. A paper-based reflectance material was used as the reference standard in this study.

A

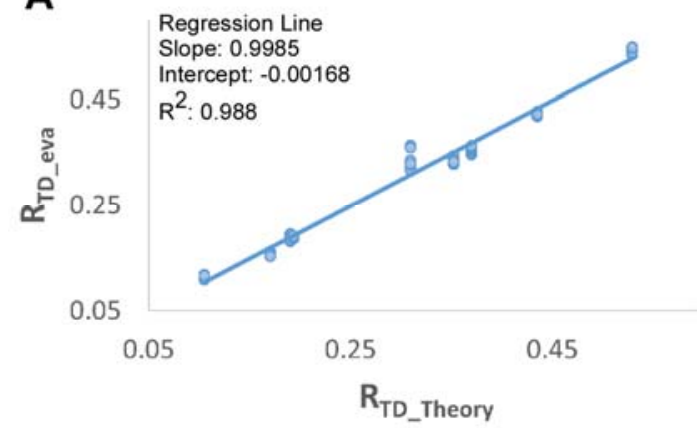

B

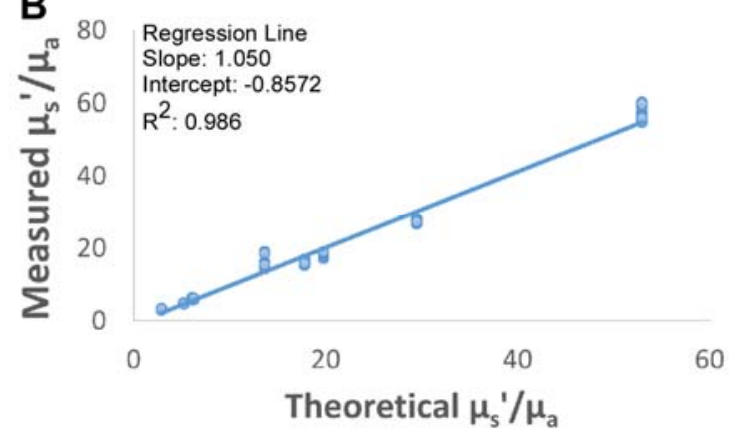

Figure 4. 11 Representative results from the evaluation study of the point spectroscopic detection modality of the hybrid system. A paper-based reflectance material was used as the reference standard in this study. A) $R_{T D \_v a}(\lambda)$ verse $R_{T D_{-} \text {theory }}(\lambda)$ at $\lambda=633 \mathrm{~nm}$. B) Measured $\mu_{s}{ }^{\prime}(\lambda) / \mu_{a}(\lambda)$ verse theoretical $\mu_{s^{\prime}}{ }^{\prime}(\lambda) / \mu_{a}(\lambda)$ at $\lambda=633 \mathrm{~nm}$. The solid lines in the figures are the linear regression lines.

The results of experimental evaluation using optical phantoms also demonstrate the high accuracy of the imaging modality of the hybrid system in terms of estimating $\mu_{\mathrm{s}}{ }^{\prime} / \mu_{\mathrm{a}}$. A strong linear relationship with slope 1 is observed between $\operatorname{RTD}_{-}$eva $(\lambda)$ and $\operatorname{RTD}_{\text {- theory }}(\lambda)$ and between measured $\mu_{\mathrm{s}}{ }^{\prime}(\lambda) / \mu_{\mathrm{a}}(\lambda)$ and theoretical $\mu_{\mathrm{s}}{ }^{\prime}(\lambda) / \mu_{\mathrm{a}}(\lambda)$ at $\lambda=500 \mathrm{~nm}$, as illustrated in Fig. 4.12. 

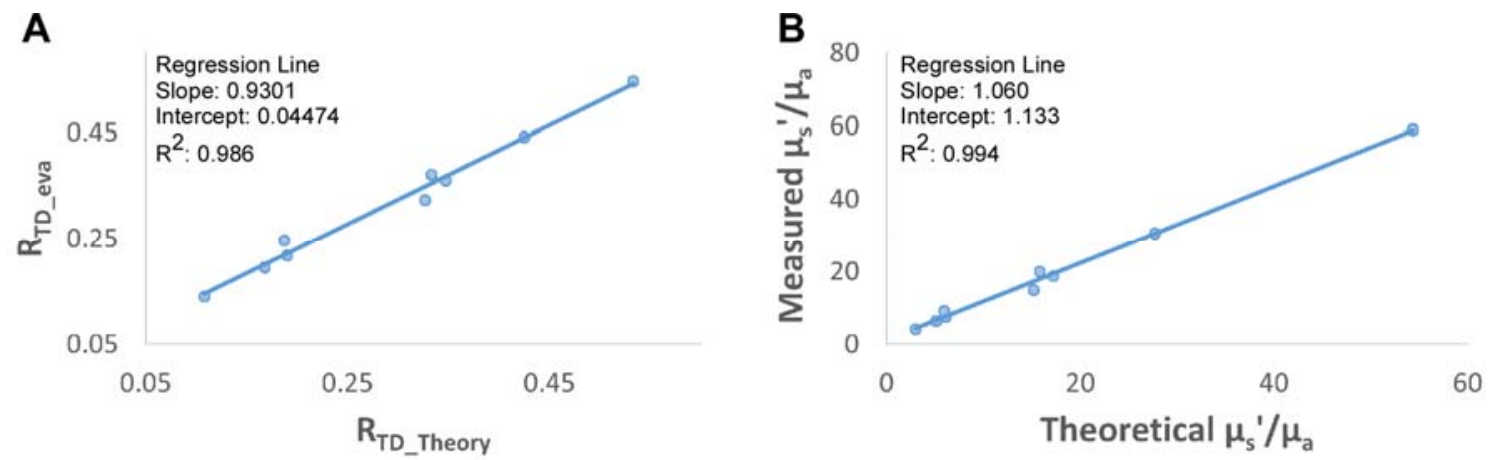

Figure 4. 12 Results from the evaluation study of the imaging modality of the hybrid system. An optical phantom was used as the reference standard in this study. A) $R_{T D_{-} \text {eva }}(\lambda)$ verse $R_{T D_{-} \text {theory }}(\lambda)$ at $\lambda=500 \mathrm{~nm}$. B) Measured $\mu_{s}^{\prime}(\lambda) / \mu_{a}(\lambda)$ verse theoretical $\mu_{s}{ }^{\prime}(\lambda) / \mu_{a}(\lambda)$ at $\lambda=500 \mathrm{~nm}$. The solid lines in the figures are the linear regression lines.

\subsection{Discussion}

The need to accurately obtain optical properties from in vivo biological tissues is largely attributed to the fact that these properties are directly linked to tissue compositional and structural characteristics, and hence provide a diagnostic potential for diseases and injuries. In this article, a unique hybrid spectroscopy imaging system was introduced to quantify optical properties of in vivo tissue. The system possesses two detection modalities: one for point spectroscopic detection and the other for imaging. The former is used when high spectral resolution is needed, while the latter is used when investigation of an area is necessary. Both detection modalities acquire relative total diffuse reflectance signals from the target in a non-contact fashion. With the assistance from a reference optical standard and a look-up table generated by the MC simulation model for photon migration, relative total diffuse reflectance measurements can be converted to absolute total diffuse reflectance and then $\mu_{\mathrm{s}} \mathrm{s} / \mu_{\mathrm{a}}$. The results of the evaluation confirm the operating principle, as well as the high accuracy of the hybrid system. 
To obtain reliable results from the hybrid spectroscopy imaging system, the following three conditions should be met: 1) the illumination intensity on the investigated subject needs to be uniform; 2) the optical properties of the investigated subject are homogeneous; 3) the anisotropy factor $\mathbf{g}$ and, more importantly, the refractive index $\mathbf{n}$ of the investigated subject should be known.

The first required condition is derived from the theoretical framework of total diffuse reflectance RTD obtained from a broad beam illumination (See 4.6 Appendix): the illumination intensity at a given point on the surface of the investigated subject should be the same as that at the point of detection in order to obtain accurate RTD. This is especially true to the illumination points that make meaningful contribution to diffuse reflectance signal measured at $\left(\mathrm{x}_{0}, \mathrm{y}_{0}\right)$. This requirement, in turn, constitutes the minimal radius of uniform illumination of the system, which can be quantitative assess using the point spread function of photon migration (i.e., radially resolved diffuse reflectance from a pencil beam illumination). According to the theory of photon migration, low $\mu_{\mathrm{s}}$ ' and $\mu_{\mathrm{a}}$ generally leads to a broader point spread function. Therefore, the minimal radius of uniform illumination increases as $\mu_{\mathrm{s}}$ ' and $\mu_{\mathrm{a}}$ decrease. In order to quantify these effects, reported optical properties of pediatric brain cortex [94] were used to simulate point spread functions at $500 \mathrm{~nm}, 600 \mathrm{~nm}$, and $700 \mathrm{~nm}$, and the simulation results are presented in Fig. 4.13. It turns out that within the visible range, the radius within which $95 \%$ of total diffuse reflectance is observed ( $\left.\mathrm{r}_{95}\right)$ is $2.6 \mathrm{~mm}$ and $5.8 \mathrm{~mm}$ for the smallest and the largest point spread function at $500 \mathrm{~nm}$, respectively. In other words, the minimal radius of uniform illumination at $500 \mathrm{~nm}$ has to exceed $5.8 \mathrm{~mm}$ in order to reduce the measurement error of total diffuse reflectance below $5 \%$. This is rather easy to attain with 
a commercial-grade surgical light and a surgical microscope light in the operating room. As expected, r95 increases significantly as the wavelength increases (i.e., $\mu_{\mathrm{s}}$ ' and $\mu_{\mathrm{a}}$ decrease). According to the simulation results, the largest $\mathrm{r}_{95}$ is found at $700 \mathrm{~nm}$ and is about $2.9 \mathrm{~cm}$. This number may not be reliable as the optical properties used may have been strongly underestimated. Nevertheless this indicates the hybrid system works better in the shorter wavelength region where $\mu_{\mathrm{s}}$ ' and $\mu_{\mathrm{a}}$ of biological tissues are high and the corresponding r95 as well as the minimal radius of uniform illumination are low.
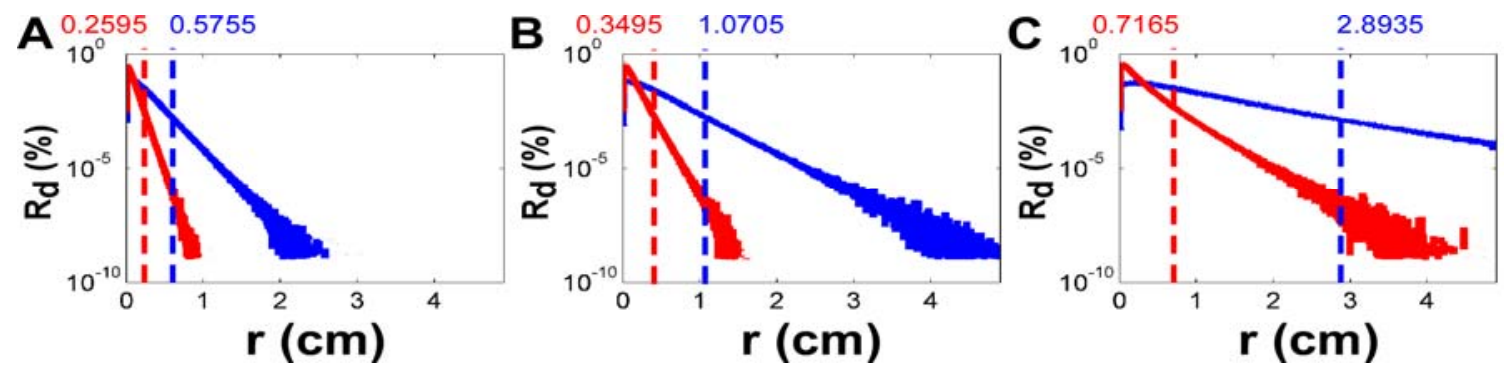

Figure 4. 13 Diffuse reflectance $\left(R_{d}\right)$ as a function of radius $(\boldsymbol{r})$ at three different wavelengths $(A-500 \mathrm{~nm}, B-600 \mathrm{~nm}$, and $C-700 \mathrm{~nm})$. The red curve demonstrated the narrowest diffuse reflectance profile, while the blue one is the widest one. The dash lines represent the cutoffs within which 95\% of total diffuse reflectance is observed. To generate these $R_{d}(\boldsymbol{r})$, the Monte Carlo simulation was carried out with the optical properties of human brain [94]. Specifically, $\boldsymbol{n}$ is $1.395, \boldsymbol{g}$ is $0.88, \mu_{a}$ is $1.6-4.5 \mathrm{~cm}^{-1}$ at $500 \mathrm{~nm}, 0.6-1.7 \mathrm{~cm}^{-1}$ at $600 \mathrm{~nm}$ and $0.07-0.19 \mathrm{~cm}^{-1}$ at $700 \mathrm{~nm}$, and $\mu_{s}$ is $83-250$ $\mathrm{cm}^{-1}$ at $500 \mathrm{~nm}, 52-228 \mathrm{~cm}^{-1}$ at $600 \mathrm{~nm}$ and $35-211 \mathrm{~cm}^{-1}$ at $700 \mathrm{~nm}$.

The second required operating condition of the hybrid system is based on the fact that the look-up table shown in Fig. 4.2 is derived using MC simulations with spatially homogenous media (i.e., constant optical properties). This assumption is used widely in the techniques measuring tissue optical properties. For the hybrid system, the minimal radius of uniform illumination discussed in the previous section defines the minimal radius of tissue homogeneity required to ensure the accurate operation of the system. To determine the minimal depth of tissue homogeneity, again the MC simulation is used in conjunction with the optical properties of pediatric brain cortex [94]. The results of the simulation indicate that the minimal depth of tissue homogeneity would be approximately 
$1.475 \mathrm{~mm}$ for $500 \mathrm{~nm}$ and $7.885 \mathrm{~mm}$ for $700 \mathrm{~nm}$. Since the hybrid system reports the average $\mu_{\mathrm{s}}{ }^{\prime} / \mu_{\mathrm{a}}$ within the volume of investigation defined by the minimal radius and depth of tissue homogeneity, the inhomogeneity will inevitably deviate the measured $\mu_{\mathrm{s}}{ }^{\prime} / \mu_{\mathrm{a}}$ from its expected value.

The simulation results presented in this article suggest that anisotropy factor $\mathbf{g}$ is not as influential as refractive index $\mathbf{n}$ to the accuracy of $\mu_{\mathrm{s}}{ }^{\prime} / \mu_{\mathrm{a}}$ estimation. However, $\mathbf{n}$ did not introduce a significant error in estimating $\mu_{\mathrm{s}}{ }^{\prime} / \mu_{\mathrm{a}}$ of the evaluation phantoms $(\mathbf{n}=$ 1.486) based on a look-up table designed for a different $\mathbf{n}(\mathbf{n}=1.395)$. This is attributed to that the reference phantom has the same refractive index $\mathbf{n}$ as the evaluation phantoms, and thus, essentially the accuracy of the relative comparison between samples with same $\mathbf{n}$ would not depend on any particular look-up table. Therefore, $\mathbf{n}$ of the investigated tissue and the reference material are a required priori knowledge for the accurate operation of the hybrid system. Refractive indices of biological tissues have been reported widely in the existing literature. For example, Jacques published a very comprehensive review on the optical properties of biological tissues [94], which could be a good reference for determining $\mathbf{n}$ of a specific tissue type and hence creating a corresponding look-up table. If $\mathbf{n}$ is not readily available, it may be estimated based on its water content using the formula depicted by Jacques [94].

The hybrid system disclosed in this article is capable of obtaining absolute total diffuse reflectance at the macroscopic and the microscopic levels and estimating a wide range of $\mu_{\mathrm{s}}{ }^{\prime} / \mu_{\mathrm{a}}(0-10000)$ efficiently. It is relatively inexpensive to construct and can be applied to various biomedical applications such as intraoperative brain tumor detection. 
The system may be easily used in the surgical operating field, and even could be integrated with a surgical microscope, without introducing any safety issues or disturbing normal clinical procedures. One significant limitation of the hybrid system is that it does not provide $\mu_{\mathrm{s}}$ ' and $\mu_{\mathrm{a}}$ separately. This limitation, however, may be circumvented by performing advanced analysis on $\mu_{\mathrm{s}}{ }^{\prime} / \mu_{\mathrm{a}}$ spectra to extract more function- and structurerelated information from biological tissues, which will be discussed in detailed formulations and verifications in the following chapter.

\subsection{Conclusion}

In this study, a hybrid spectroscopy imaging system and a simple data interpretation algorithm were introduced to quantify the optical properties $\left(\mu_{\mathrm{s}}{ }^{\prime} / \mu_{\mathrm{a}}\right)$ of a given turbid media through the measurement of total diffuse reflectance. The system is capable of acquiring total diffuse reflectance spectra over a broad wavelength range from a point on the targeted sample, as well as acquiring 2-D images at multiple wavelengths from the targeted sample. With the help of a reference material with known optical properties and a look-up table constructed using MC simulations, the system is able to convert the measured total diffuse reflectance to optical properties $\left(\mu_{\mathrm{s}}{ }^{\prime} / \mu_{\mathrm{a}}\right)$ efficiently and accurately. The effects of other optical properties, namely refractive index $\mathbf{n}$ and anisotropy factor $\mathbf{g}$, on the accuracy of the estimation algorithm were also evaluated. It was found that only alterations in refractive index could introduce significant errors to the estimation of $\mu_{\mathrm{s}}{ }^{\prime} / \mu_{\mathrm{a}}$ of the system. 


\subsection{Appendix}

Diffuse reflectance $\mathrm{R}_{\mathrm{d}}(\mathbf{r})$ defines the percentage of the incident photon energy that is diffusely reflected by a tissue sample as a function of the distance between the illumination and the observation points $\mathbf{r}$. Therefore, total diffuse reflectance $\mathrm{R}_{\mathrm{TD}}$ can be express as:

$$
R_{T D}=\int_{0}^{\infty} R_{d}(r) d r
$$

If the surface of a tissue sample is illuminated by a large beam with a uniform intensity distribution, as illustrated in Fig. 4.14A, diffuse reflectance observed from an arbitrary point $(\mathrm{x}, \mathrm{y})$ on the tissue surface will be

$$
R(x, y)=\int_{0}^{\infty} \int_{0}^{2 \pi}\left[\frac{R_{d}(r)}{2 \pi r}\right] \cdot r d \theta d r
$$

where $\mathbf{r}$ is the distance between an illumination point $\left(\mathrm{x}^{\prime}, \mathrm{y}^{\prime}\right)$ and the observation point $(\mathrm{x}, \mathrm{y})$. Let $(\mathrm{x}, \mathrm{y})=(0,0)$, then $r=\sqrt{x^{\prime 2}+y^{\prime 2}}$ and $\mathrm{R}(\mathrm{x}, \mathrm{y})$ will be the same as $\mathrm{R}$ TD. 
A

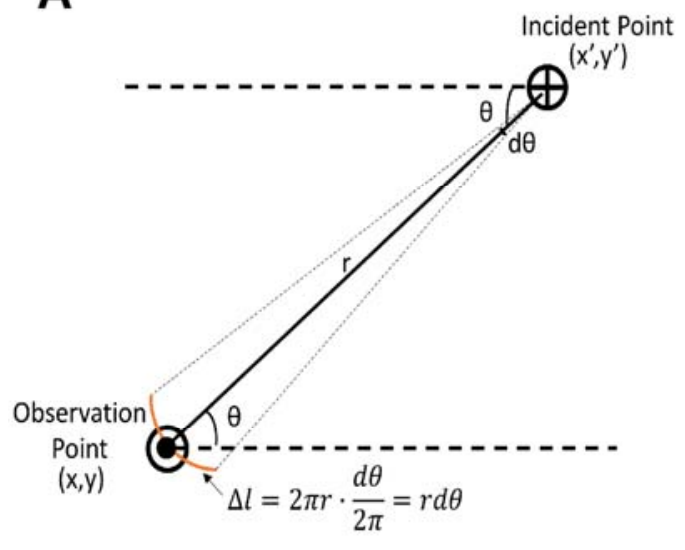

$\bigoplus$ Pencil beam illumination

B

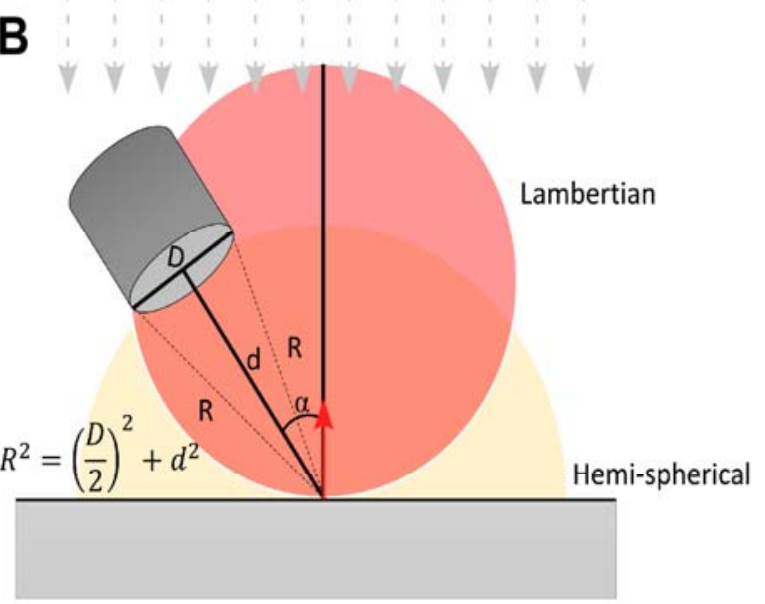

(2) Point of detection

Figure 4. 14 Graphic illustrations of (A) total diffuse reflectance measured from an arbitrary point on a tissue surface illuminated by a broad uniform beam and (B) the fraction $\boldsymbol{f}$ associated with the collection geometry.

When the collection geometry is taken into consideration, the acquired diffuse reflectance signal will be a fraction (f) of $\mathrm{R}_{\mathrm{TD}}$, as illustrated in Fig. 4.14B. The exact value of $\mathbf{f}$ will be determined by (1) the distance between the detector and the observation point $\mathbf{d}$, and (2) the lens diameter $\mathbf{D}$. That is

$$
f=\frac{2 \pi \cdot\left(d^{2}+\left(\frac{D}{2}\right)^{2}\right) \cdot\left(1-\frac{d}{\sqrt{\left(d^{2}+\left(\frac{D}{2}\right)^{2}\right)}}\right)}{\frac{1}{2} \cdot 4 \pi \cdot\left(d^{2}+\left(\frac{D}{2}\right)^{2}\right)}=1-\frac{d}{\sqrt{\left(d^{2}+\left(\frac{D}{2}\right)^{2}\right)}},
$$

where the angular distribution of the diffuse reflectance signal is assumed to be uniform (i.e., the hemi-spherical profile illustrated in Fig. 4.14B). However, Eq. (4.6) is not applicable to biological tissues because the angular distribution of diffuse reflectance 
from biological tissues usually is a Lambertian. As a result, the fraction $\mathbf{f}$ for biological tissues is calculated by

$$
f=\frac{\int_{\alpha-a \tan D / 2 d}^{\alpha+a \tan D / 2 d} I \cdot \cos (\Omega) d \Omega}{\int_{-\frac{\pi}{2}}^{\frac{\pi}{2}} I \cdot \cos (\Omega) d \Omega}=\frac{\left.\sin (\Omega)\right|_{\alpha-a \tan D / 2 d} ^{\alpha+a \tan D / 2 d}}{\left.\sin (\Omega)\right|_{-\frac{\pi}{2}} ^{\frac{\pi}{2}}}=\frac{2 \sin \alpha \cdot \cos (a \tan D / 2 d)}{2}=\frac{d}{\sqrt{d^{2}+\left(\frac{D}{2}\right)^{2}}} \sin \alpha
$$

When the observation distance and angle are maintained, $\mathbf{f}$ will be a constant. Therefore, $R_{x x x}$ measure $(\lambda)$ in Eq. (4.2) is a relative measurement of the absolute total diffuse reflectance $\mathrm{R}_{\mathrm{TD} \_\mathrm{xxx}}(\lambda)$. That is

$$
R_{\text {xxx_measure }}(\lambda)-R_{\text {dark }}(\lambda)=f \cdot R_{T D_{-} x x x}(\lambda)
$$

This relationship is applicable to both reference and evaluation measurements. Therefore, the effect of $\mathbf{f}$ will be cancelled in the ratio operation in Eq (4.2) and hence is not noticed. That is

$$
\frac{R_{\text {eva_measure }}(\lambda)-R_{\text {dark }}(\lambda)}{R_{\text {ref_measure }}(\lambda)-R_{\text {dark }}(\lambda)} \cdot R_{T D_{-} r e f}(\lambda)=\frac{f \cdot R_{T D_{-} \text {eva }}(\lambda)}{f \cdot R_{T D_{-} r e f}(\lambda)} \cdot R_{T D_{-} \text {ref }}(\lambda)=R_{T D_{-} \text {eva }}(\lambda)
$$




\section{A new algorithm for determination of hemodynamic and structural characteristics of brain tissue from total diffuse reflectance measurements}

\subsection{Introduction}

Diffuse reflectance spectroscopy (DRS) has been demonstrated capable of effectively detecting hemodynamic and structural characteristics of biological tissues in vivo; this capability has been applied to various intraoperative applications such as detecting brain tumor at the resection margin intraoperatively (see Chapter 3). However, artifacts originated from the hand motions and contact pressure could compromise the interpretation of the hemodynamic properties [46, 49], and hence, the accuracy of the tumor detection. In addition, the data acquisition procedure with the DRS system will interrupt the routine surgical procedures and require complete darkness in the operating room to avoid ambient light influence, which is quite inconvenient for the neurosurgeons and nurses. Therefore, a non-contact, motion-artifact-free and user-friendly point spectroscopic detection system was devised in-house to avoid these issues (see Chapter 4). Instead of measuring the spatial-resolved diffuse reflectance, the new measurement scheme is able to capture a fraction of the total diffuse reflectance under a wide-field uniform illumination.

Since the nature of the $\mathrm{R}_{\mathrm{TD}}$ is different from that of radially-dependent diffuse reflectance signal $R_{d}(r)$ [88], the spectral interpretation algorithms [95, 96] previously developed to extract the hemodynamic and structural properties from $\operatorname{Rd}(r)$ is no longer valid. In this study, a new spectral interpretation algorithm was developed for $\mathrm{R}_{\mathrm{TD}}$ spectra to extract the hemodynamic (hemoglobin concentration, $[\mathrm{Hb}]$, and oxygen saturation level, $\mathrm{SatO}_{2}$ ) and the structural (scattering) properties of the biological tissue. The 
accuracy and sensitivity of the spectral interpretation algorithm was evaluated both theoretically with Monte Carlo simulation. Subsequently, the system as well as the interpretation algorithm were applied to a forepaw stimulation study of Wistar rats to demonstrate its clinical utilities.

\subsection{Methods}

\subsubsection{Instrumentation}

The system developed to acquire $\mathrm{R}_{\mathrm{TD}}$ consisted of two signal acquisition modalitiesthe imaging modality and the point spectroscopic detection modality (Fig. 5.1). The prototype system utilized a white light source (DC-950H, Dolan-Jenner, Edmund Optics Inc., Barrington, NJ) for illumination during the animal experiment. The cortical surface was imaged through a zoom imaging lens (VZM 450i, EdmundOptics). The Hastings triplet achromatic lens (\#30-229, EFL 40.3 mm, Edmund Optics Inc., Barrington, NJ) collimated the image and then passed it through to a beam splitter (\#54-824, 50R/50T, Edmund Optics Inc., Barrington, NJ). The imaging modality was attached to the transmission port (T-port) of the bean splitter holder. The collimated image through the T-port were refocused onto the CCD cameras (BFLY-U3-03S2M-CS, Point Grey Research, Inc.) by another Hastings lens. The point spectroscopic detection modality was attached to the reflection port (R-port) of the beam splitter holder. The collimated image through the R-port was projected via another Hastings lens onto the $x-y$ translation stage coupled with an optical fiber (core diameter $50 \mu \mathrm{m}, \mathrm{NA}=0.2$, GIF50C, Thorlabs). The distal end of the optical fiber could be connected to either a red laser source (CPS184, $650 \mathrm{~nm}, 4.5 \mathrm{~mW}$, Thorlabs) to track the point of detection on the cortical surface, or a 
spectrometer (USB2000, Ocean Optics, Dunedin, FL) to acquire the relative RTD spectrum from a small area on the cortex. All image and spectrum acquisitions were conducted using self-developed LabVIEW programs.

As explained in previous chapter, reflectance signal obtained from any point on the image plane under the uniform illumination is equal to a fraction of the RTD generated by a pencil beam illumination. With a reference material (optical properties are known) and a look-up table (Fig. 5.2a) based on a Monte-Carlo (MC) simulation model for photon migration, the acquired relative $\mathrm{R}_{\mathrm{TD}}$ signal could be accurately converted to the ratio of absorption coefficient to reduced scattering coefficient (i.e., $\mu_{\mathrm{s}}{ }^{\prime} / \mu_{\mathrm{a}}$ ) [88].

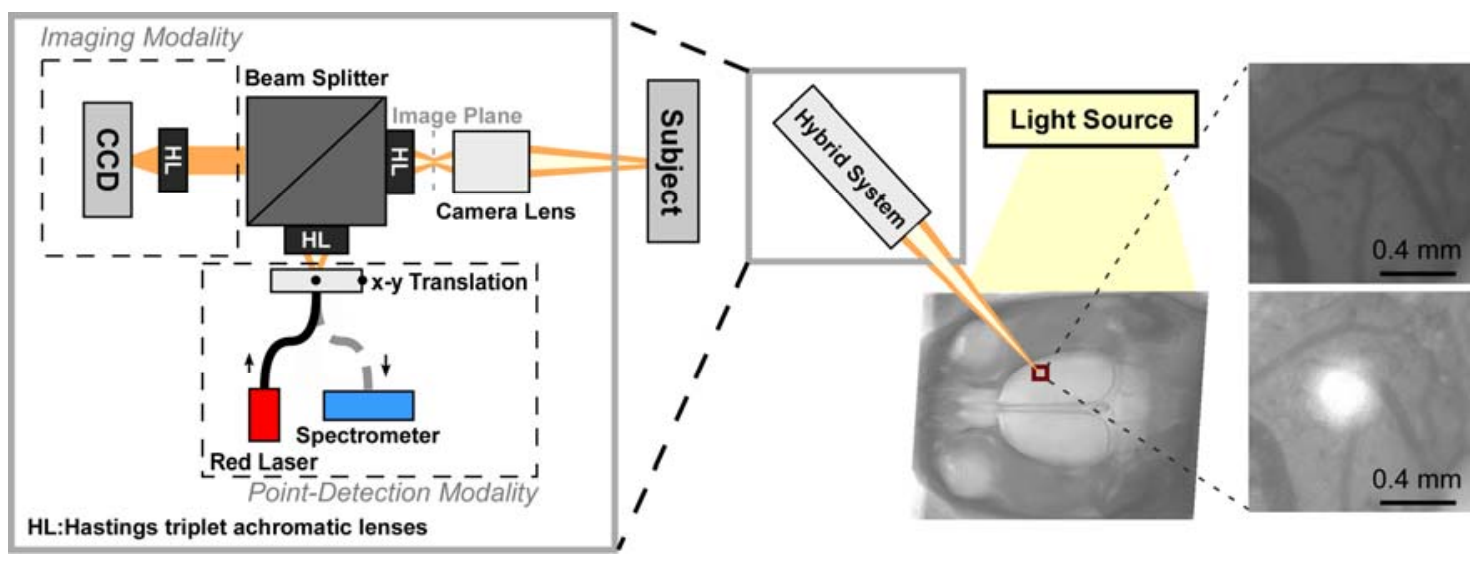

Figure 5. 1 Schematics of the non-contact $R_{T D}$ spectroscopy system

\subsubsection{Spectral interpretation algorithm}

With the estimated $\mu_{\mathrm{s}}{ }^{\prime} / \mu_{\mathrm{a}}$ values, more information could be extrapolated with certain formulations with regard to the absorption and scattering properties of brain cortex: Since hemoglobin is the major absorber of the brain tissue within the visible wavelength range and hence, the absorption coefficient could be expressed as 


$$
\mu_{a}(\lambda)=[H b] \cdot\left(\varepsilon_{\text {oxy }}(\lambda) \cdot \operatorname{SatO}_{2}+\varepsilon_{\text {deoxy }}(\lambda) \cdot\left(1-\operatorname{SatO}_{2}\right)\right) \cdot 2.303 / 64500
$$

where $[\mathrm{Hb}]$ is the total hemoglobin concentration in the unit of $\mathrm{g} /$ liter, $\varepsilon_{\text {oxy }}(\lambda)$ and $\varepsilon_{\text {deoxy }}(\lambda)$ are the wavelength-dependent molar extinction coefficients of oxy- and deoxyhemoglobin [34], respectively, $\mathrm{SatO}_{2}$ is the oxygen saturation level, 64500 is the gram molecular weight of hemoglobin and 2.303 is the conversion factor. Furthermore, the reduced scattering coefficient could be determined by a simple power law

$$
\mu_{s}^{\prime}(\lambda)=A \cdot\left(\frac{\lambda}{500}\right)^{-B}
$$

where $\lambda$ is in $\mathrm{nm}, \mathrm{A}$ is a scaling factor and $\mathrm{B}$ is the scattering power. Factor $\mathrm{A}$ is equal to the value of $\mu_{\mathrm{s}}{ }^{\prime}(\lambda=500 \mathrm{~nm})$, which might be time-dependent. $B$ characterizes the wavelength dependence of $\mu \mathrm{s}^{\prime}$. Eq. (5.2) is good for predicting the scattering properties within the visible wavelength range [94]. Based on the above mentioned conditions, $\mu_{\mathrm{s}}{ }^{\prime} / \mu_{\mathrm{a}}$ could be expressed as

$$
\frac{\mu_{s}{ }^{\prime}(\lambda)}{\mu_{a}(\lambda)}=\frac{A \cdot(\lambda / 500)^{-B}}{[H b] \cdot\left(\varepsilon_{\text {oxy }}(\lambda) \cdot \operatorname{SatO}_{2}+\varepsilon_{\text {deoxy }}(\lambda) \cdot\left(1-\operatorname{SatO}_{2}\right)\right) \cdot 2.303 / 64500} .
$$

Since the molar extinction coefficients of oxy- and deoxy-hemoglobin are equal (i.e., $\left.\varepsilon_{\text {oxy }}\left(\lambda_{i}\right)=\varepsilon_{\text {deoxy }}\left(\lambda_{i}\right)\right)$ at the isosbestic wavelengths $\left(\lambda_{i}=500,529,545,570\right.$, and $584 \mathrm{~nm}$, approximately), the value of $\mathrm{SatO}_{2}$ will not have any influence on $\mu_{\mathrm{a}}$ at these wavelengths. Hence, the following non-linear model could be obtained 


$$
\left(\begin{array}{c}
\frac{\mu_{s}{ }^{\prime}\left(\lambda_{1}\right)}{\mu_{a}\left(\lambda_{1}\right)} \\
\frac{\mu_{s}{ }^{\prime}\left(\lambda_{2}\right)}{\mu_{a}\left(\lambda_{2}\right)} \\
\vdots \\
\frac{\mu_{s}^{\prime}\left(\lambda_{i}\right)}{\mu_{a}\left(\lambda_{i}\right)}
\end{array}\right)=\frac{A}{[H b]}\left(\begin{array}{c}
\frac{\left(\lambda_{1} / 500\right)^{-B}}{C\left(\lambda_{1}\right)} \\
\frac{\left(\lambda_{2} / 500\right)^{-B}}{C\left(\lambda_{2}\right)} \\
\vdots \\
\frac{\left(\lambda_{i} / 500\right)^{-B}}{C\left(\lambda_{i}\right)}
\end{array}\right)
$$

where the wavelength-dependent constant $\mathrm{C}\left(\lambda_{\mathrm{i}}\right)$ is

$$
C\left(\lambda_{i}\right)=\frac{\varepsilon_{\text {oxy }}\left(\lambda_{i}\right)+\varepsilon_{\text {deoxy }}\left(\lambda_{i}\right)}{2} \cdot 2.303 / 64500 .
$$

A simple logarithm transformation on Eq. (5.4) could yield a linear model:

$$
\log \left(\begin{array}{c}
\frac{\mu_{s}{ }^{\prime}\left(\lambda_{1}\right)}{\mu_{a}\left(\lambda_{1}\right)} \\
\frac{\mu_{s}{ }^{\prime}\left(\lambda_{2}\right)}{\mu_{a}\left(\lambda_{2}\right)} \\
\vdots \\
\frac{\mu_{s}{ }^{\prime}\left(\lambda_{i}\right)}{\mu_{a}\left(\lambda_{i}\right)}
\end{array}\right)=\log \left(\begin{array}{c}
\frac{A}{[H b] \cdot C\left(\lambda_{1}\right)} \\
\frac{A}{[H b] \cdot C\left(\lambda_{2}\right)} \\
\vdots \\
\frac{A}{[H b] \cdot C\left(\lambda_{i}\right)}
\end{array}\right)+(-B) \cdot \log \left(\begin{array}{c}
\frac{\lambda_{1}}{500} \\
\frac{\lambda_{2}}{500} \\
\vdots \\
\frac{\lambda_{i}}{500}
\end{array}\right) .
$$

Applying the least-square regression method to Eq. (5.6) (Fig. 5.2b), A/[Hb] and B could be estimated, respectively. With an estimation of $A /[\mathrm{Hb}]$ and $B$, the $C(\lambda)$, which is the wavelength-dependent total hemoglobin extinction coefficient, at all other non-isosbestic wavelength could be computed by

$$
C(\lambda)=\frac{\mu_{s}{ }^{\prime}(\lambda)}{\mu_{a}(\lambda)} \cdot\left(\frac{A}{[H b]}\right)^{-1} \cdot\left(\frac{\lambda}{500}\right)^{B} .
$$


Eq. (5.7) is an inverse method to determine the total hemoglobin extinction coefficient spectrum $C(\lambda)$. By definition, $C(\lambda)$ can be determined with the knowledge of $\varepsilon_{\text {oxy }}(\lambda), \varepsilon_{\operatorname{deoxy}}(\lambda)$ and $\mathrm{SatO}_{2}$ :

$$
C(\lambda)=\left(\varepsilon_{\text {oxy }}(\lambda) \cdot \operatorname{SatO}_{2}+\varepsilon_{\text {deoxy }}(\lambda) \cdot\left(1-\operatorname{SatO}_{2}\right)\right) \cdot 2.303 / 64500 .
$$

For a given $\mathrm{SatO}_{2}$ level, normalized $\mathrm{C}(\lambda)$ spectrum (i.e., $\mathrm{C}(\lambda) / \mathrm{C}(530 \mathrm{~nm})$ ) has a unique spectral profile (i.e., number of peaks and peak locations, as shown in Fig. 5.2c). Using the peak locations of the normalized $\mathrm{C}(\lambda)$, a look-up table to estimate the absolute $\mathrm{SatO}_{2}$ values was established (see Fig. 5.2c). Note the look-up table was constructed by manually varying the value of $\mathrm{SatO}_{2}$ in Eq. (5.8) to generate the corresponding normalized $C(\lambda)$ spectrum. The featuring peak location of a normalized $C(\lambda)$ spectrum could be determined by the "findpeaks" function in Matlab. The absolute value of $\mathrm{SatO}_{2}$ could be immediately determined with Eq. (5.7) and the look-up table for $\mathrm{SatO}_{2}$ (Fig. 5.2c), following the estimation of $\mathrm{A} /[\mathrm{Hb}]$ and $\mathrm{B}$ in Eq. (5.6). 

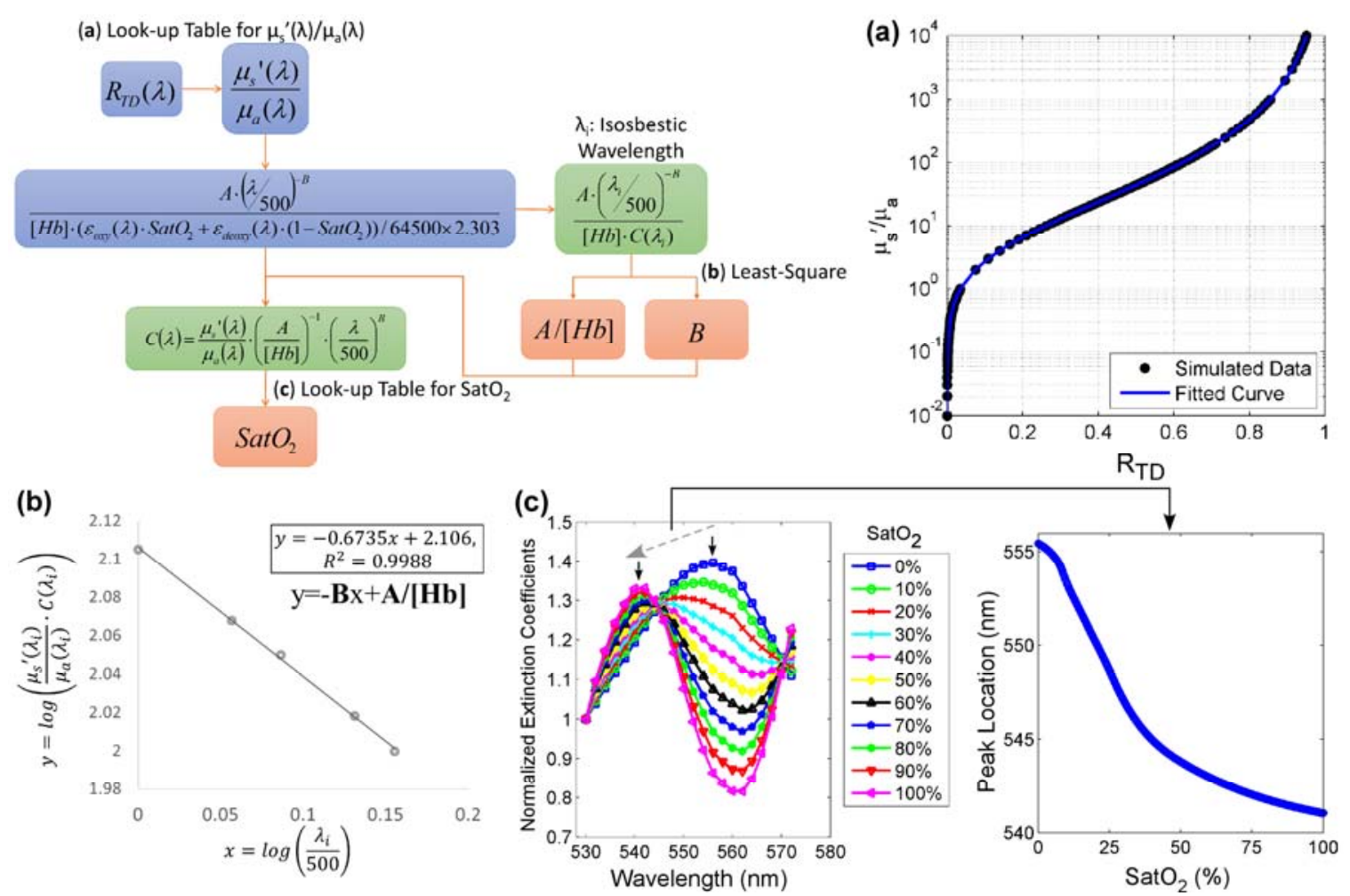

Figure 5. 2 Algorithm to estimate hemodynamic and structural characteristics

\subsubsection{Validation based on Monte Carlo simulation}

In order to evaluate the efficacy of the algorithm in estimating $\mathrm{A} /[\mathrm{Hb}], \mathrm{B}$ and $\mathrm{SatO}_{2}$ directly from total diffuse reflectance spectrum, a validation study based on Monte Carlo simulation was designed. A Matlab program was developed to generate 50 sets of random values for the parameters $\mathrm{A}, \mathrm{B},[\mathrm{Hb}]$, and $\mathrm{SatO}_{2}$, within the ranges depicted in Table 5.1, and then to compute their corresponding optical properties $\mu_{\mathrm{a}}(\lambda)$ and $\mu_{\mathrm{s}}{ }^{\prime}(\lambda)$ using Eq. (5.1) and Eq. (5.2). These 50 sets of optical properties, along with refractive index $\mathbf{n}=1.395$ and anisotropy factor $\mathbf{g}=0.88$, were applied to a Monte Carlo (MC) simulation model for photon migration to generate total diffuse reflectance ( $\mathrm{R}_{\mathrm{TD}}$ ), assuming the simulated tissue phantom was infinitely thick $(\sim 100 \mathrm{~cm})$ and illuminated by a pencil beam perpendicularly. All optical properties used were selected in accordance to those of 
human brain tissue summarized by Jacques et al. [94]. The wavelength range covered in the MC simulation was also listed in Table 5.1, with an increment of $2 \mathrm{~nm}$.

Table 5. 1 Ranges of parameters used in the Monte Carlo simulation to generate $R_{T D}$ spectra

\begin{tabular}{|c|c|c|}
\hline Parameter & Minimum & Maximum \\
\hline $\mathrm{A}\left(\mathrm{cm}^{-1}\right)$ & 10 & 30 \\
\hline $\mathrm{B}$ (a.u.) & 0.5 & 2.5 \\
\hline$[\mathrm{Hb}](\mathrm{g} / \mathrm{liter})$ & 22.5 & 60 \\
\hline $\mathrm{SatO} 2(\%)$ & 0 & 100 \\
\hline$\lambda(\mathrm{nm})$ & 450 & 600 \\
& & \\
\hline
\end{tabular}

The simulated RTD spectra between $450 \mathrm{~nm}$ and $600 \mathrm{~nm}$ were applied in the spectral interpretation algorithm written in Matlab to estimate the corresponding values of $\mathrm{A} /[\mathrm{Hb}], \mathrm{B}$ and $\mathrm{SatO}_{2}$ using the interpretation methods described above. The estimated $\mathrm{A} /[\mathrm{Hb}], \mathrm{B}$ and $\mathrm{SatO}_{2}$ subsequently compared with the original values used in the MC simulation in terms of the absolute percentage error $|\Delta \mathrm{E}|$

$$
|\Delta E|=\left|\frac{v_{2}-v_{1}}{v_{1}}\right| \times 100 \%,
$$

where $v_{1}$ is the original value and $v_{2}$ is the estimated one. In addition, for the purpose of evaluating the effects of noises on the spectra interpretation algorithm, different levels 
$(0.1 \%, 0.5 \%, 1 \%$ and $5 \%)$ of uniform-distributed random noises were added to the simulated RTD spectra by

$$
R_{T D}(\lambda) \pm \frac{1}{2} \cdot R_{T D}(\lambda) \cdot \text { randomnumber }
$$

where the random number is within the range of corresponding noise level. Parameters $\mathrm{A} /[\mathrm{Hb}], \mathrm{B}$ and $\mathrm{SatO}_{2}$ were also estimated based on the noisy $\mathrm{R}_{\mathrm{TD}}$ spectra, and compared with pre-set values using Eq. (5.9).

In practice, the influence of random noise is usually reduced by averaging multiple measurements. To demonstrate the importance of averaging repeated measurements for parameter estimation, random noise at the same level $(0.1 \%, 0.5 \%, 1 \%$ or $5 \%)$ was generated and added to the simulated RTD spectra, according to Eq. (5.10). The noise addition process was repeated 10 times, which yielded 10 noisy $\operatorname{RTD}(\lambda)$. The 10 noisy $\operatorname{RTD}(\lambda)$ at a given noise level were averaged to yield an averaged spectra for spectral interpretation. The resulting parameter estimations were compared with the ones from the noisy spectra without averaging in terms of $|\Delta \mathrm{E}|$.

\subsubsection{Forepaw stimulation of Wistar rats}

Forepaw stimulation on rodents is often used to induce significant hemodynamic responses, including cerebral blood flow, cerebral blood volume and oxygen partial

pressure, within the somatosensory cortex for forelimb (S1FL) for the purpose of investigating the neurovascular coupling mechanism. It offers a great opportunity for this study to verify whether the proposed new measurement scheme and the accompanying 
spectral interpretation algorithm are capable of detecting those stimulation-induced hemodynamic variations in the S1FL cortex.

The animal study protocols were approved by the Institutional Animal Care and Use Committee (IACUC) at Florida International University (Approval Number: 13-065) and carried out in full compliance with federal, state and local regulations and laws. All procedures were performed with anesthetized rats to minimize their suffering. Eight normal Wistar rats were used in this study. Four of them were over 1 year old, and the other four were around 2-6 months old at the time of experiment. Rat's body temperature $\left(\sim 37^{\circ} \mathrm{C}\right)$, heart rate $(200-300$ beats per minute) and respiration rate $(<50$ breath per minute) were monitored continuously, using PowerLab 8/35 data acquisition device and LabChart software (AD Instruments), to assure the level of anesthesia remained stable throughout the entire surgical procedure and recordings.

Rats were fixed on the stereotaxic stage with skull and dura removed on top of the somatosensory cortex for forelimb (S1FL) under infusion of $2 \%$ isoflurane with $1 \mathrm{~L} / \mathrm{min}$ oxygen (14 psi). The location of S1FL was determined based on the rat brain atlas [97]. Non-conductive paraffin oil drops (O121-1, Mineral Oil, Light, Fisher Scientific) were applied to the craniotomy to prevent the cortical surface from dehydration during the recordings. The contralateral forepaw was stimulated using bipolar needle-electrodes placed subcutaneously. Prior to the forepaw stimulation, $0.25-\mathrm{mg} / \mathrm{kg}$ dexdomitor was intraperitoneally injected to sedate the rats. Subsequently, the isoflurane was reduced to $0.25 \%\left(1 \mathrm{~L} / \mathrm{min} \mathrm{O}_{2}, 14 \mathrm{psi}\right)$. Stimulation pulses (10-ms pulse width) were delivered by an isolated pulse stimulator (Model 2100, 110V, $60 \mathrm{~Hz}, \mathrm{~A}-\mathrm{M}$ Systems; $~ 2-\mathrm{mA}$ amplitude at 
$3 \mathrm{~Hz}$ ). A repetitive block-design paradigm was employed with each block consisting of an $\mathrm{ON}$ period of 5, 16, or 30 seconds, during which stimulation pulses were triggered, and an OFF period (i.e., resting state) of 30, 44, or 50 seconds, respectively.

\subsubsection{Acquisition of point spectroscopic detection modality}

A paper-based reference was calibrated with an optical phantom prior to the animal experiments. The optical properties of the optical phantom are known, and therefore, its theoretical $\mathrm{R}_{\mathrm{TD}}$ ref could be estimated from the look-up table of $\mathrm{R}_{\mathrm{TD}}$ versus $\mu_{\mathrm{s}}{ }^{\prime} / \mu_{\mathrm{a}}$, as described in previous chapter. The theoretical RTD_paper was estimated by

$$
R_{T D_{-} \text {paper }}(\lambda)=\frac{R_{\text {paper_measure }}(\lambda)-R_{\text {dark }}(\lambda)}{R_{\text {ref_measure }}(\lambda)-R_{\text {dark }}(\lambda)} \cdot R_{T D_{-} \text {ref }}(\lambda),
$$

where $\mathrm{R}_{\text {paper_measure }}$ and $\mathrm{R}_{\text {ref_measure }}$ were the spectra measured from the paper reference and optical phantom under the wide-field illumination using the point spectroscopic detection modality of the system, $R_{\text {dark }}$ was obtained when the illumination was turned off.

During the animal experiment, the paper reference was placed on top of the craniotomy to obtain the averaged Rpaper_measure spectra based on 10 -second continuous spectral acquisition with an integration time of $250 \mathrm{~ms}$ before and after the forepaw stimulation paradigm. Total diffuse reflectance spectra from the S1FL cortex $\mathrm{R}_{\text {cortex_measure }}$ were continuously acquired with an integration time of $250 \mathrm{~ms}$ during the forepaw stimulation paradigm. Dark measurement $R_{\text {dark }}$ was also attained. The time-dependent absolute total diffuse reflectance spectra of the S1FL cortex RTD_cortex was calculated by 


$$
R_{T D_{-} \text {cortex }}(\lambda, t)=\frac{R_{\text {cortex_measure }}(\lambda, t)-R_{\text {dark }}(\lambda)}{R_{\text {paper_measure }}(\lambda)-R_{\text {dark }}(\lambda)} \cdot R_{T D_{-} \text {paper }}(\lambda) .
$$

The corresponding values of $\mu_{\mathrm{s}}{ }^{\prime} / \mu_{\mathrm{a}}$ of the S1FL cortex could be derived from RTD_cortex spectra instantaneously using the look-up table of RTD versus $\mu_{\mathrm{s}}{ }^{\prime} / \mu_{\text {a. }}$. The event-related parameters $\mathrm{A} /[\mathrm{Hb}], \mathrm{B}$ and $\mathrm{SatO}_{2}$ could be subsequently estimated using the proposed spectral interpretation algorithm. 


\subsection{Results}
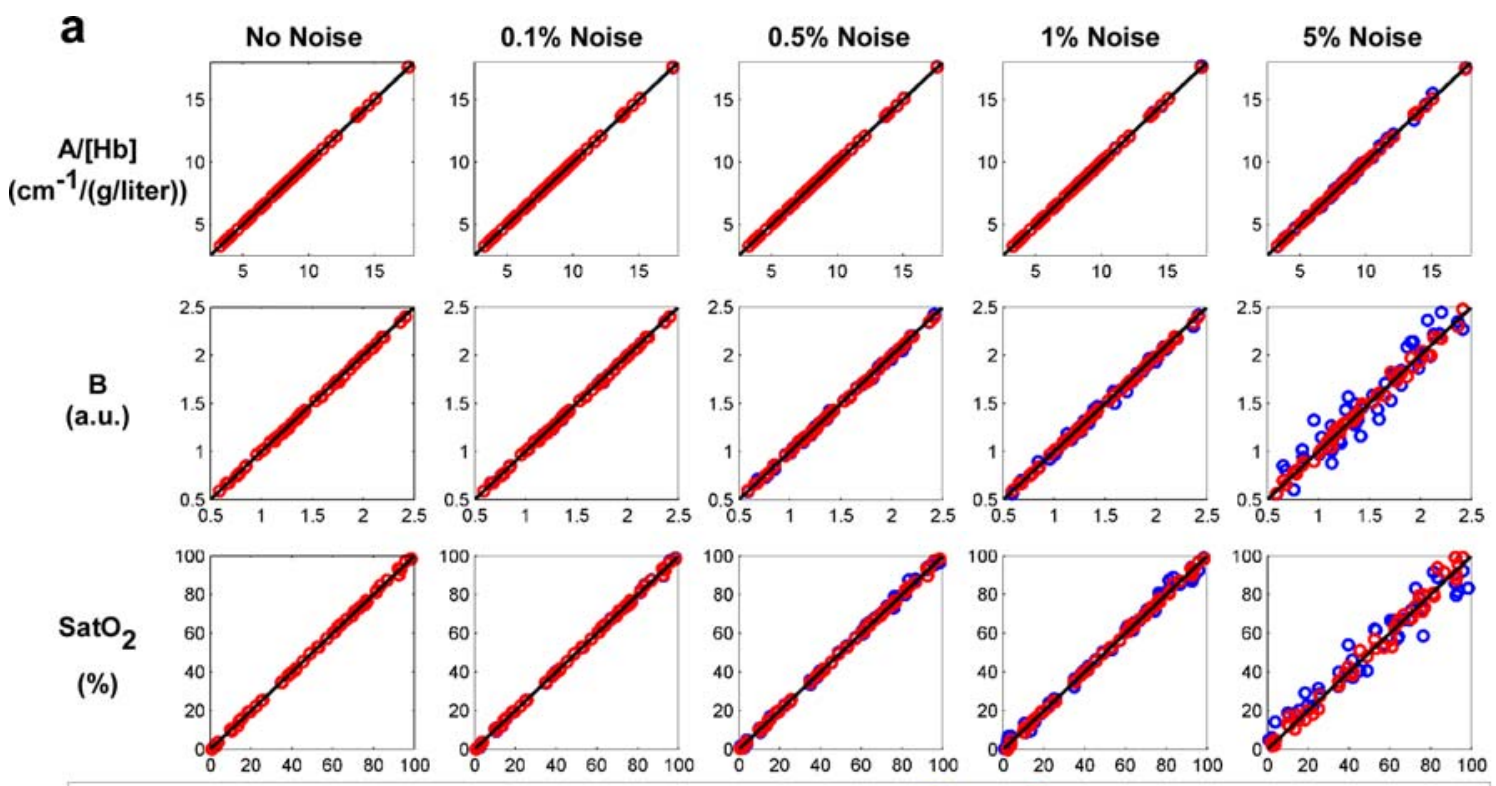

Vertical Axis: Estimated Value; Horizontal Axis: Original Value;

$\mathbf{O N}=1 ; O \mathrm{~N}=10 ;-$ Slope=1, Intercept=0.
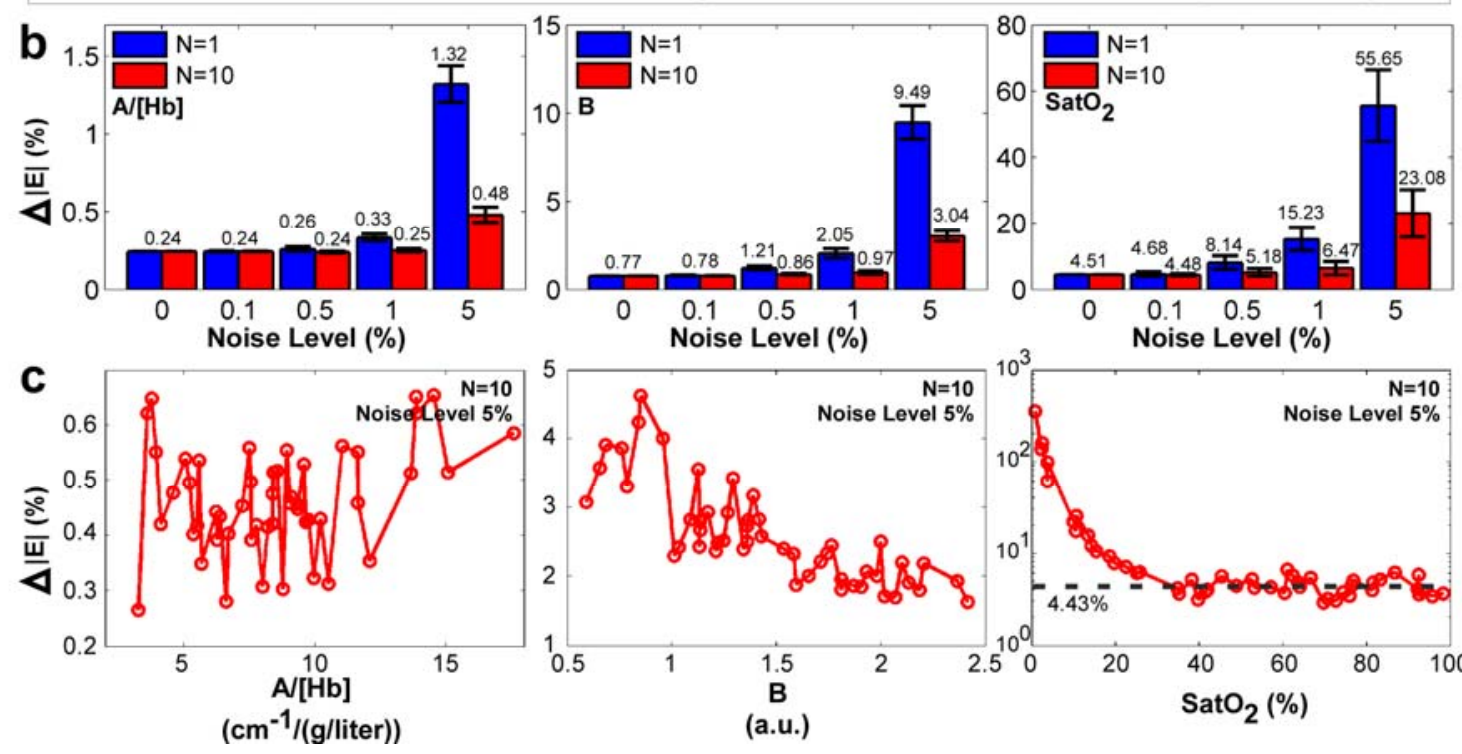

Figure 5. 3 Validation of the proposed RTD spectral interpretation algorithm using Monte Carlo simulation.

The simulated $\mathrm{R}_{T D}$ spectra with or without noises were processed using the $\mathrm{R}_{\mathrm{TD}}$ spectral interpretation algorithm to estimate the indicative parameters $\mathrm{A} /[\mathrm{Hb}], \mathrm{B}$ and $\mathrm{SatO}_{2}$. The discrepancies between estimated and the theoretical values of these indicative parameters were displayed using scatter plots, as shown in Fig. 5.3a. The accuracy of the 
$\mathrm{R}_{\mathrm{TD}}$ spectral interpretation algorithm was clearly demonstrated by the fact that all data points in the scatter plots fit the line with a slope of 1 very well.

The effect of the averaging process on the performance of the $\mathrm{R}_{\mathrm{TD}}$ spectral interpretation algorithm is shown in Fig. 5.3b. When the averaged noisy RTD spectra were used as the input, $|\Delta \mathrm{E}|$ of each indicative parameter was markedly reduced (Fig. 5.3b in red). This effect is especially prominent when the noise level is high. However, $|\Delta \mathrm{E}|$ of $\mathrm{SatO}_{2}$ estimation at the noise level of $5 \%$ was still very high $(\sim 23 \%)$ even when the averaged noisy spectra were used. It should be noted that the noise effect is not a constant for the entire range of $\mathrm{SatO}_{2}$; the $|\Delta \mathrm{E}|$ was within an acceptable range $(<5 \%)$ when the theoretical value of $\mathrm{SatO}_{2}$ was over 30\%, as shown in Fig. 5.3c.

The event-related hemodynamic responses induced by forepaw stimulation in rats were measured using the spectroscopy imaging system and then estimated by the spectral interpretation algorithm (Fig. 5.4 and Fig. 5.5). Significant variations in all three indicative parameters $\mathrm{A} /[\mathrm{Hb}], \mathrm{B}$ and $\mathrm{SatO}_{2}$ under different durations of forepaw stimulation were observed both from the rats over 1 year old and those less than 6 months old. Interestingly, the amplitude of the variations in three indicative parameters obtained from the younger rats (Fig. 5.5) were much higher than those from the older rats (Fig. 5.4). In addition, the baseline values of $\mathrm{SatO}_{2}$ from the older rats' $\mathrm{S} 1 \mathrm{FL}$ cortex were significantly lower than those from the younger rats (Fig. 5.6). 


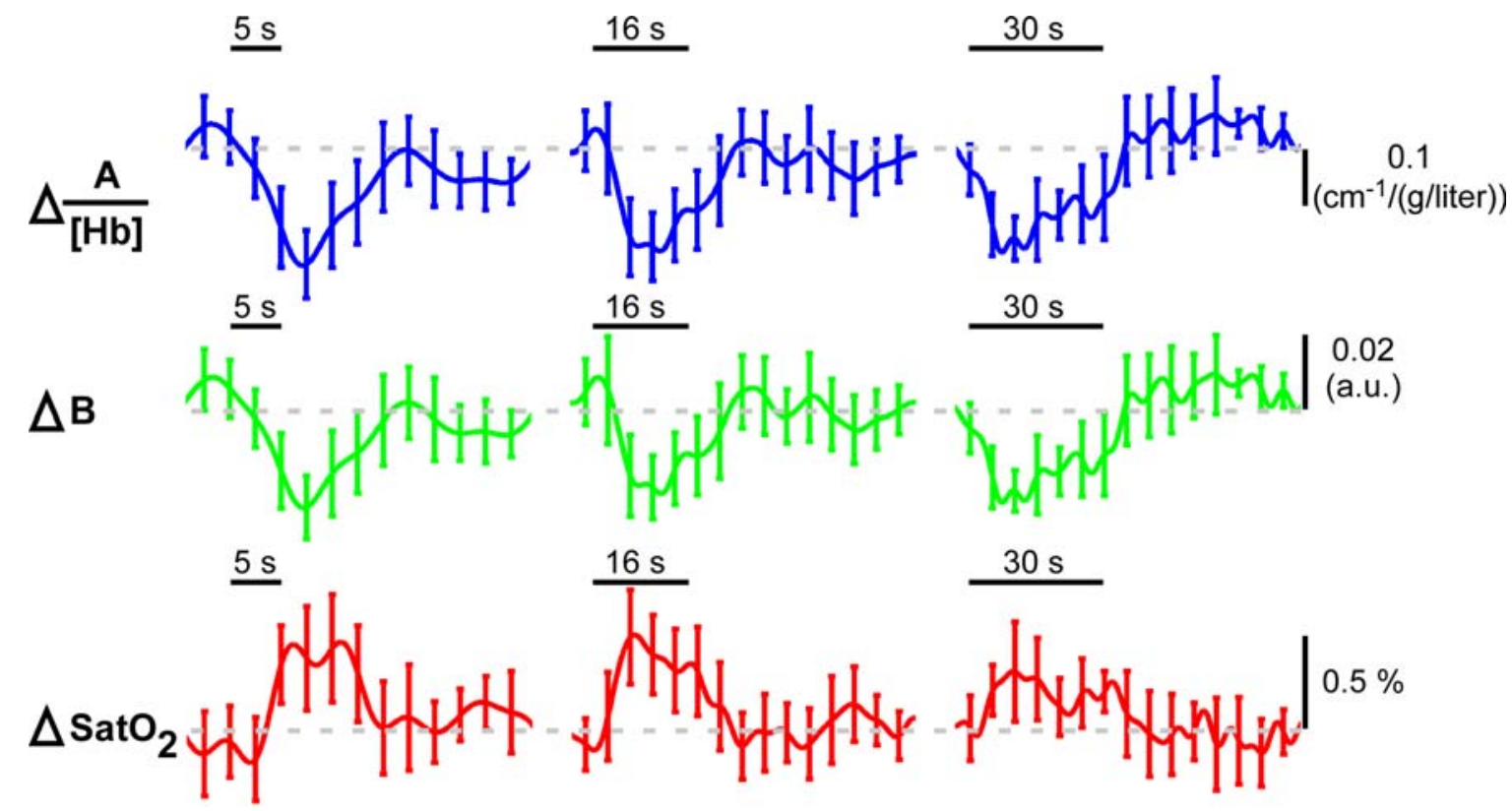

Figure 5. 4 Results from the forepaw stimulation in old rats $(N=2)$. The time histories of the indicative parameters were obtained from nine events induced by 5-second stimulation, 18 events by 16-second stimulation, and 8 events by 30-second stimulation. The solid lines are the averaged time histories. The error bars represent the standard deviations. The gray dash-line marked the baseline of each indicative parameter.

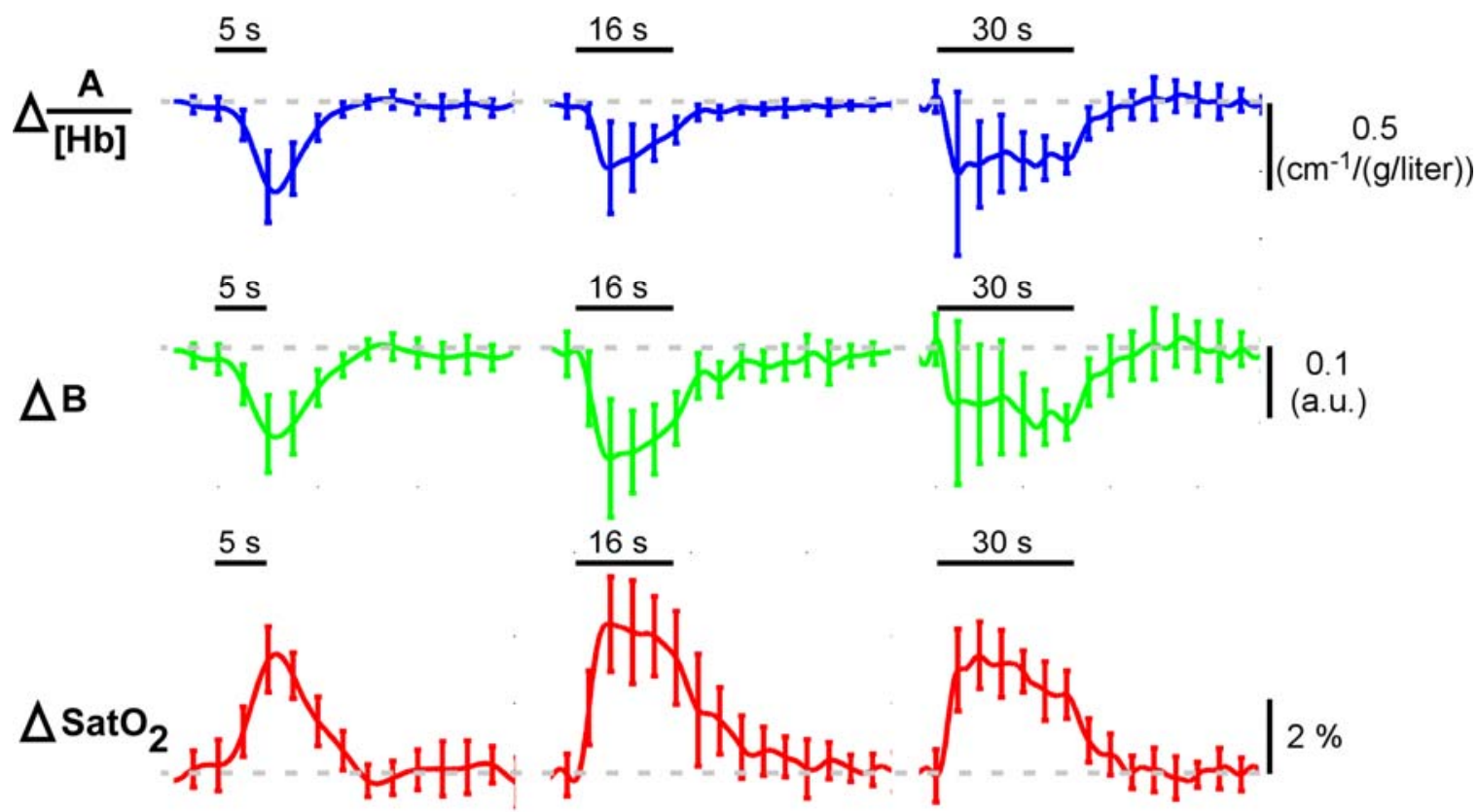

Figure 5. 5 Results from the forepaw stimulation in younger rats $(N=2)$. The time histories of the indicative parameters were obtained from 13 events induced by 5-second stimulation, 12 events by 16-second stimulation and 15 events by 30-second stimulation. The solid lines are the averaged time histories. The error bars represent the standard deviations. The gray dash-line marked the baseline of each indicative parameter. 


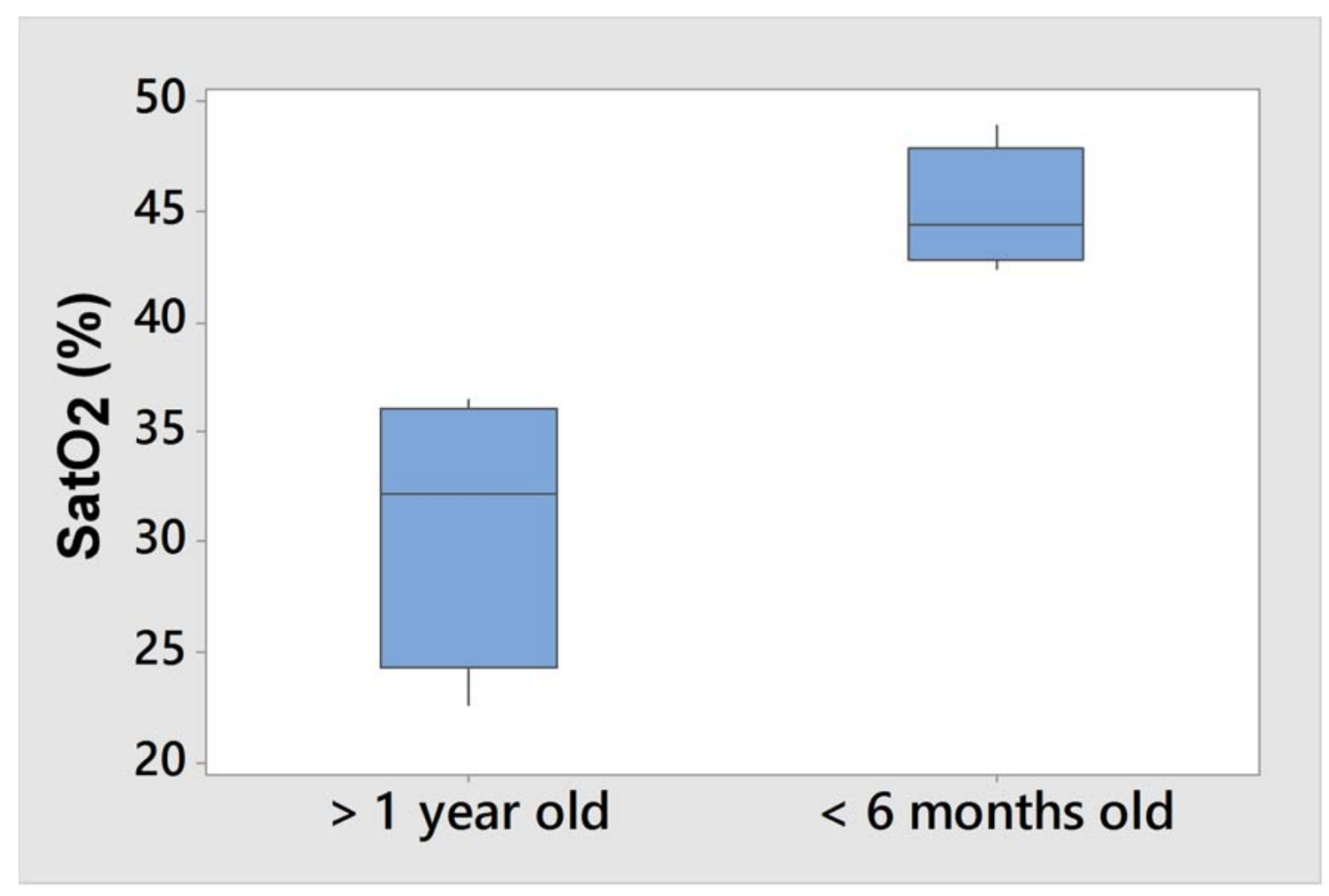

Figure 5. 6 Baseline $\mathrm{SatO}_{2}$ from 4 old rats and 4 young rats

\subsection{Discussion}

In this study, a new spectral interpretation algorithm was proposed to retrieve three indicative parameters related to the hemodynamic and structural characteristics of biological tissue based on total diffuse reflectance spectrum between 500 to $580 \mathrm{~nm}$. Currently, the most popular spectral interpretation algorithm applied on multi-wavelength optical data to estimate the hemodynamics variations is based on modified Beer-Lambert law [98-100]. The scattering properties are usually assumed to be constant in order to obtain the desired hemodynamic information. A priori knowledge about the baseline hemoglobin concentration and oxygen saturation level are also needed to determine the mean optical path length in the modified Beer-Lambert law. These assumptions would introduce bias to quantify the real hemodynamic characteristics. Furthermore, these 
algorithms are not capable of producing absolute $\mathrm{SatO}_{2}$. In contrast, the spectral interpretation algorithm proposed in this study does not rely on any assumption about the baseline values of each indicative parameters. In addition, it does not assume the scattering properties are constant. The most important advantage of this algorithm is that it could objectively provide the absolute value of $\mathrm{SatO}_{2}$, instead of a relative estimation based on pre-set values.

Another advantage of the proposed measurement scheme and spectral interpretation algorithm is that it utilizes optical data within the short wavelength region (i.e., 500-580 $\mathrm{nm}$ ), which leads to a small volume of investigation (VOI). This, in terms, reduced the effects of tissue inhomogeneity on the estimation accuracy of the indicative parameters. It has been demonstrated in previous chapter that the VOI at $700 \mathrm{~nm}$ is markedly greater than the one at $500 \mathrm{~nm}$, due to much lower absorption and scattering coefficients of biological tissues in the near infrared wavelength region.

In general, the proposed spectral interpretation algorithm could handle a moderate level $(<5 \%)$ of noises. By averaging the raw spectra, the estimation error could be significantly reduced. However, caution should be taken when estimated $\mathrm{SatO}_{2}$ is below $30 \%$, since it is more susceptible to the accuracy degradation induced by noises, as demonstrated in the validation study based on MC simulation. This susceptibility may be originated from the look-up table for $\mathrm{SatO}_{2}$ estimation; the increase in the full width half max of the peak at the low $\mathrm{SatO}_{2}$ levels makes the peak location detection highly inaccurate, with the presence of the strong noises. 
To validate the utility and the efficacy of the new measurement scheme and the proposed algorithm in practice, an animal study with forepaw stimulation was carried out. Significant hemodynamic responses were expected to be observable in S1FL whenever the electrical current stimulation was introduced to the forepaw of the rat. The estimated indicative parameters depicted significant variations related to the stimulation, which was reproducible and clearly demonstrated the capability of the system in differentiating the variations in the hemodynamic $\left(\mathrm{SatO}_{2}\right.$ and $\left.\mathrm{A} /[\mathrm{Hb}]\right)$ and structural $(\mathrm{A} /[\mathrm{Hb}]$ and $\mathrm{B})$ characteristics. The variations observed in the scattering power B might be a result of the increase of blood flow in the studied tissue, though it requires further investigation to gain insight to the underlying mechanism. Meanwhile, this observation contradicts the assumption regarding constant scattering properties usually used in applying the modified Beer-Lambert law $[99,100]$. This might indicate those results obtained based on this constant scattering property assumption need to be carefully reinterpreted.

Although it was not the intended scope of this study, the system was also able to detect a significant age-related difference in (1) the baseline levels of $\mathrm{SatO}_{2}$ and (2) the magnitudes of the stimulation induced variation in $\mathrm{SatO}_{2}$ among the studied rats. This phenomenon may be attributed to the aging of the brain. It has been demonstrated that even in healthy human subjects, the oxygen consumption in the brain will decrease with age $[101,102]$, which could result from a combined effects of neuronal loss, cellular biological impairment and functional deafferentation. Such effects were inferred to underlie or reflect the age-related cognitive changes [102]. 
The performance of the new measurement scheme and spectral interpretation algorithm in differentiating brain tumor from normal tissue in vivo remains to be investigated and compared with the probe-based DRS system in the future, which could have tremendous value in clinical applications. In addition, they could be utilized to perform the intraoperative monitoring of tissue $\mathrm{SatO}_{2}$ during, for example, a bypass surgery as well. It will also be of great interest to include them in the preclinical studies of neurovascular coupling, in combination with other neuroimaging modalities, such as laser Doppler flowmetry and electrophysiology. The current instrumentation design could also facilitate the experimental set-up of these multi-modal imaging studies.

\subsection{Conclusion}

In this study, the feasibility of using a non-contact point spectroscopic detection system to estimate the hemodynamic and structural characteristics of biological tissue, through the relative total diffuse reflectance measurements, was successfully demonstrated. The spectral interpretation algorithm is capable of handling moderate levels of random noise imposed on the measured data, as investigated based on MC simulation. In a rat forepaw stimulation study, the proposed measurement scheme and spectral interpretation algorithm were able to detect significant hemodynamic and structural variations in the somatosensory cortex for forelimb caused by the electrical current stimulation. Both theoretical and experimental validations indicated that the new measurement scheme and the spectral interpretation algorithm could uncover the intrinsic hemodynamic and structural characteristics of biological tissue effectively, which could have tremendous value in both clinical and preclinical investigations. 


\section{Intraoperative optical mapping of epileptogenic cortices in pediatric patients}

\subsection{Introduction}

Complete removal of epileptogenic brain areas that are responsible for seizure onset offers patients with refractory epilepsy the chance of being seizure free. This act, however, must be balanced with the preservation of the eloquent cortical areas to reduce the postoperative morbidity [26]. Many evaluation technologies have been used during the preoperative evaluation phase for epilepsy surgeries, including electroencephalogram (EEG), (functional) magnetic resonance imaging ((f)MRI), positron emission tomography (PET) and single-photon emission computed tomography (SPECT). Together, they provide the general location but not the exact boundaries of seizure-inducing brain areas. The usefulness of all this information degrades during the course of surgery because of brain shift and deformation that is invariably induced by the loss of cerebrospinal fluid $[12,13]$. Therefore, additional intraoperative evaluations are always needed to finalize the surgical plan and guide the surgery.

Electrocorticography $(\mathrm{ECoG})$ has been demonstrated as a valuable intraoperative tool to identify eloquent cortical areas, as well as epileptic brain areas (i.e., responsible for either ictal or interictal discharges). It can be used for chronic recordings; however, the required additional surgery for electrode implantation elevates the risks of hemorrhage, infection and cerebral edema [26]. Intraoperative MRI (iMRI) and fMRI (ifMRI) may play crucial roles in future epilepsy surgery, as they enable a maximum extent of resection despite the lesion's proximity to eloquent brain cortex and fiber tracts which, in turn, leads to favorable seizure reduction outcomes and acceptable neurological deficit 
rates [16]. However, the use of iMRI or ifMRI demands an extremely high standard of infrastructure and maintenance. As a result, only a limited number of hospitals and research institutes have the financial and technical capabilities to offer these technologies for routine patient care. In addition, the functional mapping of ifMRI relies on the selection of hemodynamic response functions, which could compromise the accuracy of localization [17].

Dynamic intrinsic optical imaging (DIOI) is generally considered a technical alternative to ifMRI, and is believed to hold great scientific potential that could not only improve the interpretations of fMRI data, but also provide more detailed understanding about the cortical micro-environment [13]. It has been suggested that DIOI is capable of identifying the epileptogenic cortex during the ictal episodes [55], but it is not practical to implement such a localization technique in an intraoperative setting, because seizure attacks are required. Additionally, when used for functional mapping, DIOI requires external neuronal stimulations, such as electrocortical stimulation [52] and peripheral stimulation $[53,54]$. The dependence on external stimulations requires delicate control of anesthetic administration to maintain the patient's consciousness, which could introduce additional risks to the surgery, especially when patients are children.

In a recent pilot study, the feasibility of using DIOI to identify the epileptogenic cortex and eloquent areas in pediatric epilepsy patients under anesthesia was explored [103]. Unique hemodynamic low-frequency oscillations (LFOs, below $0.1 \mathrm{~Hz}$ ) with unknown sources were observed within both epileptogenic and eloquent cortical areas during the interictal periods. Unfortunately, it was unclear how to distinguish the 
epileptogenic cortex from the eloquent areas solely based on the presences of LFOs. Therefore, in the current study, two methods were developed to analyze the cortical hemodynamic LFOs acquired by DIOI on pediatric epilepsy patients for the purpose of delineating the epileptogenic cortex from eloquent areas intraoperatively. The first method was derived using seed-based correlation, feature clustering and Granger causality analysis. The second method has a physiological basis, and relies on stochastic modeling and machine learning method (i.e., support vector machine, SVM). An independent ECoG analysis was performed by neurologists at Nicklaus Children's Hospital, and its results were used as the reference to confirm the localizations of epileptic and eloquent cortical areas. Finally in this study, vasculature features of ECoGdetermined epileptogenic and normal cortical areas were investigated by a third method utilizing a static digital imaging modality.

\subsection{Methods}

\subsubsection{Patients Selection}

This in vivo study was approved by the Western Institutional Review Board. Eleven patients $(<18$ years old $)$ with lesional epilepsy undergoing one/two-stage epilepsy surgery were chosen by their neurosurgeons (Dr. Sanjiv Bhatia and Dr. John Ragheb) at Nicklaus Children's Hospital. Informed consent was obtained from each patient and their parents prior to the surgery. Patients' demographic and clinical information was summarized in Table 6.1. The neurosurgeons were not aware of the results presented in this study at the time of surgery. 
Table 6. 1 Patients' demographic and clinical information

\begin{tabular}{|l|l|l|l|l|l|}
\hline Patient & Gender & Age (yo) & Stage & Craniotomy & Pathology \\
\hline 1 & Male & 14 & 2 & L F P & Tuberous sclerosis \\
\hline 2 & Female & 17 & 2 & R F T & Probable FCD \\
\hline 3 & Female & 7 & 2 & R & Type 2A FCD \\
\hline 4 & Male & 12 & 2 & L & n/a \\
\hline 5 & Male & 16 & 2 & R F T & Rasmussen encephalitis \\
\hline 6 & Female & 13 & 2 & L F P T & Cavernous malformation \\
\hline 7 & Female & 15 & 2 & L F T & $\begin{array}{l}\text { Type 2A FCD; mild hippocampal } \\
\text { gliosis }\end{array}$ \\
\hline 8 & Male & 8 & 2 & L T & Cellular glial tumor; favor FCD \\
\hline 9 & Male & 9 & 2 & R F T & Type 2A FCD \\
\hline 10 & Female & 12 & 2 & L T O & Gliosis \\
\hline 11 & Male & 2 months & 1 & R F T & FCD \\
\hline
\end{tabular}

yo: years old; L: Left; R: Right; F: Frontal; T: Temporal; O: Occipital; P: Parietal; FCD: Focal cortical dysplasia.

\subsubsection{Optical Data Acquisition}

Images at $500 \mathrm{~nm}$ and $700 \mathrm{~nm}$ were acquired simultaneously and continuously from the exposed cortical surface intraoperatively, using a DOSIS system developed in-house (Song et al., 2012). The exposed cortex was illuminated by the surgical light in the operating room and imaged through a Nikon dSLR lens (Nikon AF 28-80mm f/3.5-5.6 D Lens with Aperture Ring). Images were recollimated and then split into two branches by a dichroic mirror (\#49-471, Edmund Optics), which has a transmission wavelength range of 400-595 $\mathrm{nm}$ and a reflection wavelength range of 640-750 $\mathrm{nm}$. Two CCD cameras (DMK 21AU04, The Imaging Source Europe GmbH) were attached to the holder of the dichroic mirror; one at the transmission port $(\mathrm{Cam} T)$ and the other at the reflection port 
(CamR). CamT recorded image through a $500 \mathrm{~nm}$ band-pass filter (\#65-149, Edmund Optics), Camr through a $700 \mathrm{~nm}$ band-pass filter (\#88-012, Edmund Optics). Both cameras were synchronized using external trigger provided by a function generator. In each image acquisition sequence, at least 1000 frames were acquired by each camera at a rate of 5 frames per second. The imaging system was controlled by a LabVIEW program via an IEEE 1394a interface. 


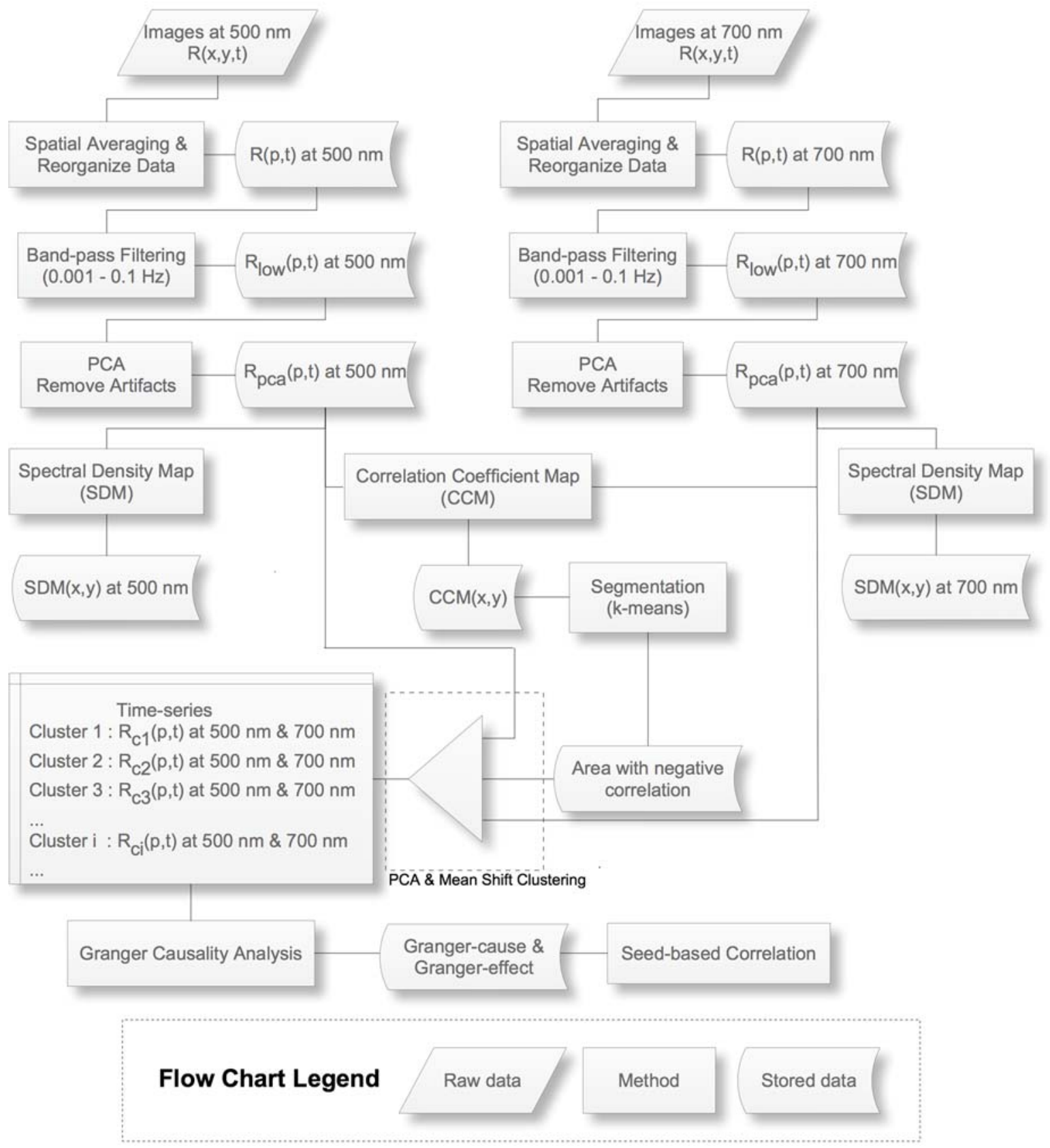

Figure 6. 1 Flow chart of the image analysis procedures (Method 1). PCA: principal component analysis.

During the DIOI study, the patient was kept still and his/her physiological conditions kept stable under normal anesthesia. A list of anesthetic agents and other surgery-related information were summarized in Supplementary Table 6.1. 


\subsubsection{Optical Data Analysis (Method 1)}

All data analyses were performed with Matlab programs developed in-house following the steps shown in Fig. 6.1. Images from both cameras were co-registered and cropped to show the same exposed cortex area at two wavelengths and smoothed using a mean filter with a 3-by-3 square window. Time series of each pixel $\mathrm{R}(\mathrm{x}, \mathrm{y}, \mathrm{t})$ was analyzed both in the time domain and the frequency domain. Since LFOs were of interest in this study, time series $R(p, t)$, where $p=(x, y)$, was filtered using a band-pass FIR filter $(\sim 0.001-0.1 \mathrm{~Hz})$, which yielded $\mathrm{R}_{\text {low }}(\mathrm{p}, \mathrm{t})$. The artifacts in $\mathrm{R}_{\text {low }}(\mathrm{p}, \mathrm{t})$ were removed using the principal component analysis (PCA) method, which produced $\mathrm{R}_{\mathrm{PCA}}(\mathrm{p}, \mathrm{t})$. The power spectral density map (SDM, $\sim 0.001-0.1 \mathrm{~Hz}$ ) was generated using $\mathrm{R}_{\mathrm{pca}}(\mathrm{p}, \mathrm{t})$ at each wavelength. Correlation coefficient map (CCM) was generated by calculating the correlation coefficient between $\mathrm{R}_{\mathrm{pca}}(\mathrm{p}, \mathrm{t})$ at $500 \mathrm{~nm}$ and that at $700 \mathrm{~nm}$ at each pixel. Pixels showing negative correlation, which often appeared as focal groups, were extracted using k-means segmentation; $R_{p c a}(p, t)$ at both wavelengths of these pixels were subsequently classified, using the mean shift clustering method, into multiple clusters based on their temporal profiles. This classification was performed without any a priori knowledge about the number of existing clusters. $R_{p c a}(p, t)$ in each cluster was then denoted as $\mathrm{R}_{\mathrm{ci}}(\mathrm{p}, \mathrm{t})$, where subscript $\mathbf{i}$ stands for the cluster number. A Granger causality toolbox (Luo et al., 2013) for non-stationary signals was applied to $\mathrm{R}_{\mathrm{ci}}(\mathrm{p}, \mathrm{t})$ at $500 \mathrm{~nm}$ to determine the directed influences among these clusters. If $\mathrm{R}_{\mathrm{ci}}(\mathrm{p}, \mathrm{t})$ from region $\mathbf{A}$ has a directed influence on $\mathrm{R}_{\mathrm{ci}}(\mathrm{p}, \mathrm{t})$ of region $\mathbf{B}$, region $\mathbf{A}$ would be denoted as Granger-cause (G-cause) while region B Granger-effect (G-effect). When the directions of influences were identified, averaged time series $R_{c i}(p, t)$ at the G-causes and G-effects were used as 
reference (i.e., seed), respectively, to calculate the seed-based correlation coefficients with $R_{p c a}(p, t)$ from the other pixels within the field of view (FOV) at each wavelength.

\subsubsection{Electrocorticography (ECoG) Acquisition and Analysis}

For patients undergoing two-stage epilepsy surgery, the optical imaging acquisition was performed prior to the ECoG study. The placement of the ECoG microelectrode arrays was determined by the results of the pre-operative evaluations using scalp EEG, MRI/fMRI, PET and/or SPECT, which was not influenced by the results of DIOI data analysis. After the first-stage surgery, the electrical activities of the target cortex of these patients were monitored for at least one week to identify the brain areas producing the ictal/interictal spikes. The final decision on the area of resection was determined by the ECoG results in conjunction with those from the neuro-imaging studies. Once the surgery plan was finalized, these patients underwent the second stage of the surgery to remove the electrode array and the epileptogenic brain areas. For patients undergoing one-stage epilepsy surgery, the optical imaging acquisition was also performed prior to the confirmatory ECoG study, which was carried out to determine the resection margin.

Neurologists at Nicklaus Children's Hospital analyzed all ECoG data and provided information with regard to the localizations of eloquent and epileptic cortical areas. The eloquent areas are defined as cortical areas consistently related to a given function; e.g., sensory or motor. The epileptogenic cortex is regarded as the area of cortex indispensable for seizure onset. Areas generating interictal spikes and discharges are also generally considered epileptic. DIOI data analyses in the previous section were conducted without any knowledge about the results from ECoG study. Later, the ECoG study results and the 
actual area of surgical resection were used as gold standards to define the epileptogenic and eloquent cortical areas.

\subsubsection{Stochastic modeling and machine learning (Method 2)}

Based on the absorption spectra of oxy- and deoxy-hemoglobin, DIOI data at $500 \mathrm{~nm}$ primarily reflect changes in cerebral blood volume (CBV). DIOI data at $700 \mathrm{~nm}$, on the other hand, reflect variations in deoxy-hemoglobin concentration [Deoxy-Hb] relative to CBV. Since both parameters are known to be affected by neuronal activities [104], investigating the relationship between the DIOI data at $500 \mathrm{~nm}$ and $700 \mathrm{~nm}$ would provide insights into the interplay between $\mathrm{CBV}$ and $\mathrm{SatO}_{2}$ under the influence of both neuronal activities and metabolisms. This, in terms, could offer a window of opportunity to separate epileptogenic from eloquent cortex using parameters that characterize the interplay between $\mathrm{CBV}$ and $\mathrm{SatO}_{2}$, To verify this hypothesis, $\mathrm{R}_{\mathrm{ci}}(\mathrm{p}, \mathrm{t})$ at both wavelengths with negative correlations from epileptogenic and eloquent cortical areas, as defined by the outcomes of the ECoG studies, were applied to an autoregressive model with an exogenous source (ARX):

$$
y_{t}=\phi_{0}+\sum_{i=1}^{p} \phi_{i} y_{t-i}+\sum_{i=1}^{r} \psi_{i} u_{t-i}+\varepsilon_{t}
$$

where $\mathbf{u}$ is $R_{c i}(p, t)$ at $500 \mathrm{~nm}$ (related to CBV) as the exogenous source, $\mathbf{y}$ is $R_{c i}(p, t)$ at $700 \mathrm{~nm}$ (reflecting variations in $\mathrm{SatO}_{2}$ ) as the output of this stochastic system, $\phi$ and $\psi$ are the coefficients for variable $\mathbf{y}$ and $\mathbf{u}$, respectively, $\mathbf{p}$ and $\mathbf{r}$ are the order of the series of $\mathbf{y}$ and $\mathbf{u}$, respectively, and $\boldsymbol{\varepsilon}$ is a white noise. Although this data-driven model is not strictly following any biophysics-based mechanism, the impulse response functions 
(IRFs) of this model, to a certain extent, could indirectly show the relationship between $\mathrm{CBV}$ and $\mathrm{SatO}_{2}$, and hence contain useful features to differentiate eloquent from the epileptogenic cortex. Therefore, a support vector machine (SVM), a common machine learning method used in predictive modeling for clinical decision-making, was employed with quadratic programming to train the computer to identify the differences between the IRFs obtained from epileptogenic and eloquent cortical areas. SVM has primarily been employed in EEG analysis for seizure detection [105-107]. It has also been used as a diagnosis tool in anatomical and functional imaging studies [108-110]. Here, Rci(p,t) obtained from the epileptogenic and eloquent cortical areas were split into two parts, one was used as the training data set and the other as a testing data set. Upon obtaining the estimated IRFs from each data set, Haar wavelet decomposition was employed to extract unique features from IRFs. The averaged coefficients of approximation at level 3 and the averaged coefficients of details at level 2 and 3 in the Haar wavelet decomposition [111] were scaled (i.e., standard deviation $=1$ ) and used in SVM as the features for training and testing purposes. Finally, the performance of the SVM with different orders of ARX model (p and r: 5-40) was quantified in terms of accuracy, sensitivity, specificity, and the area under the receiver operating characteristics (ROC) curve, in order to find the optimal approach to differentiate the epileptogenic cortical areas from the eloquent ones.

\subsubsection{Static Digital Imaging and Analysis of the Vasculature Abnormalities (Method 3)}

In addition to DIOI, digital color images of the exposed cortex were also acquired using a commercial-grade digital SLR camera (Sony $\alpha 100$ ) during the surgery. ImageJ (http://imagej.nih.gov/ij/) was used to perform edge detections on these images to 
determine the vessel network. Several area of interest (AOI) with the same dimensions were selected from the ECoG defined the epileptic area and the normal area within the same brain lobe. A histogram was generated for each AOI using its 8-bit post-processed image (the edges of the blood vessels highlighted); which indirectly quantified the vessel density information. Finally, the histograms of the AOIs from epileptogenic cortex were statistically compared against those from normal cortex.

\subsection{Results}

Eleven patients undergoing epilepsy surgery were enrolled in this study, and their demographic and clinical information is provided in Table 6.1. An optical imaging study was performed twice in three of these patients; each of these two studies possessed a unique view angle to the exposed cortex. Therefore, the analysis results from a total of fourteen image sequence sets are presented.

Fig. 6.2 shows an example image acquired with the DIOI system (Fig. 6.2A, at 700 nm) versus a second image captured using a commercial-grade digital camera (Fig. 6.2B). Following the DIOI image acquisitions, another picture of the exposed cortical surface was taken with the ECoG microelectrode array placed on top (Fig. 6.2C). Neurologists at Nicklaus Children's Hospital documented the locations of epileptic and eloquent cortical areas using the ECoG electrode number. With these three images, the results from the DIOI data analyses could be easily compared with those of ECoG data analyses. 


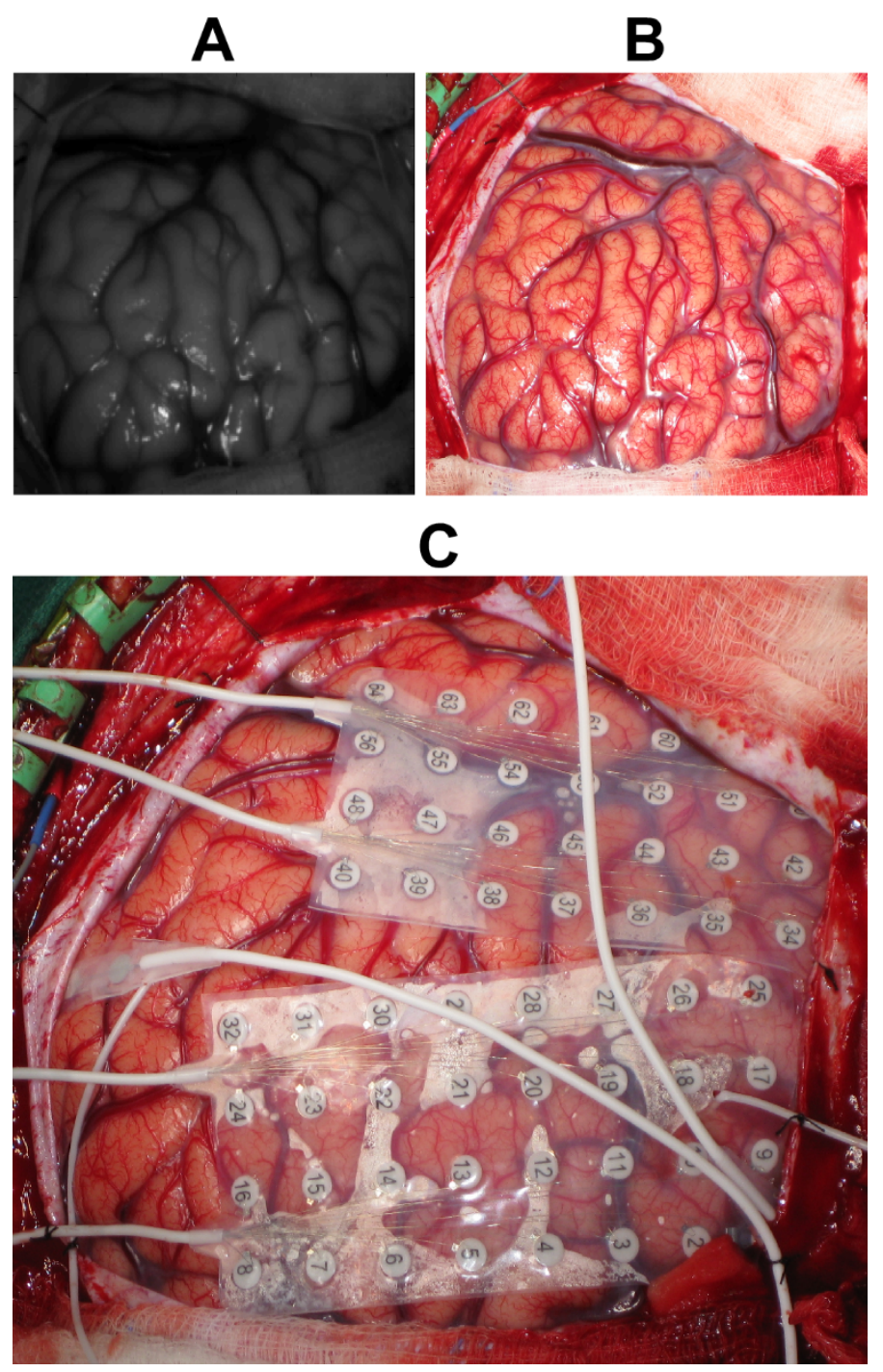

Figure 6. 2 An example of images acquired by dynamic intrinsic optical imaging (DIOI) system (A) and commercial dSLR camera (B). An image was also taken with dSLR camera after the placement of electrocorticography (ECoG) electrode array $(C)$, for the purpose of post-analysis comparison between DIOI and ECoG analyses.

\subsubsection{Correlation, clustering and Granger causality (Method 1)}



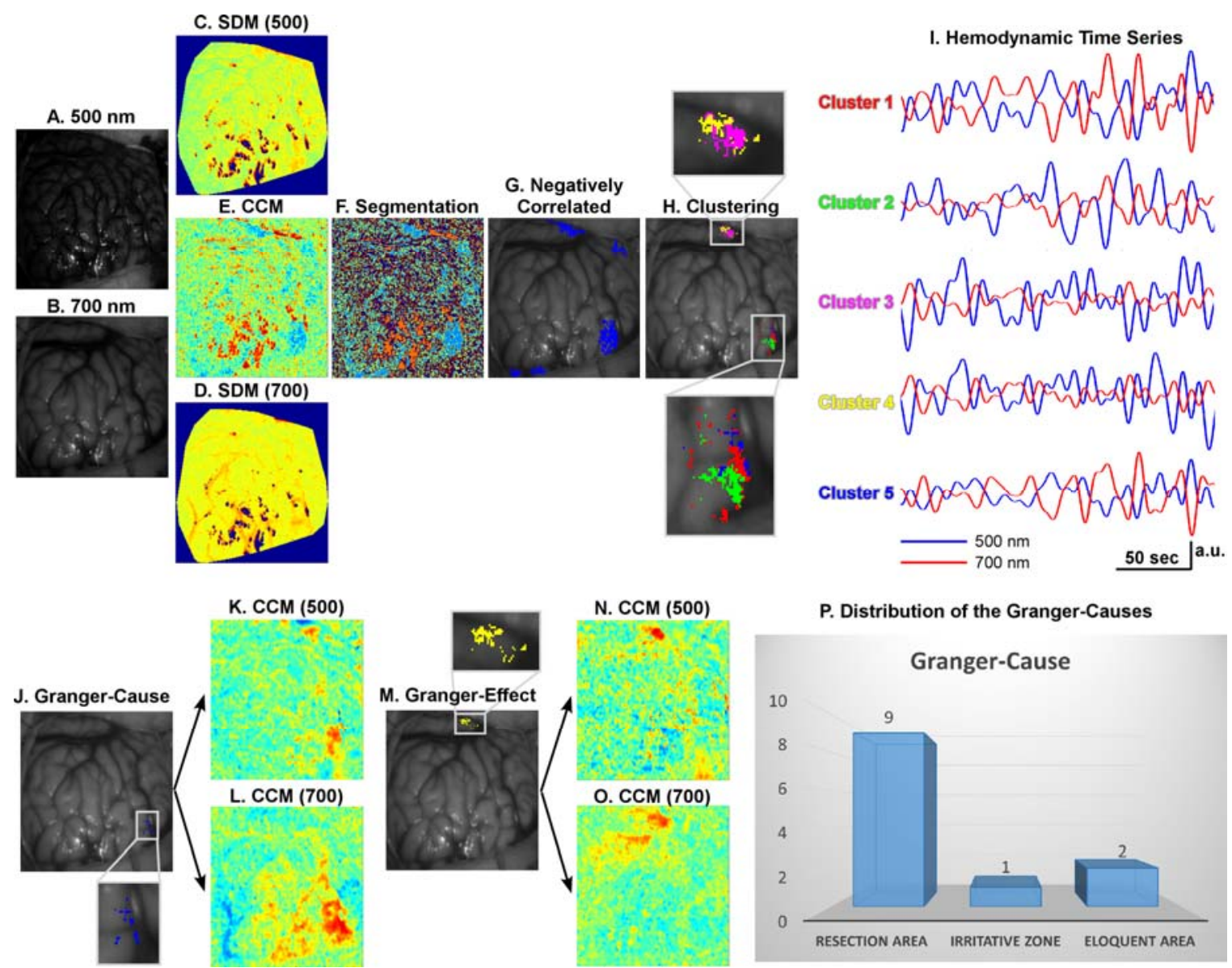

Figure 6. 3 Analysis of dynamic intrinsic optical imaging (DIOI) data (Method 1). (A) and (B) were the raw images obtained by DIOI at $500 \mathrm{~nm}$ and $700 \mathrm{~nm}$, respectively. Spectral density maps (SDMs) at $500 \mathrm{~nm}(C)$ and $700 \mathrm{~nm}(D)$ show the power of hemodynamic low-frequency oscillations (LFOs). Correlation coefficient map (CCM) in (E) was obtained by calculating the correlation coefficient between LFOs at both wavelengths. K-means segmentation $(F)$ was performed to isolate those regions with negative correlation $(G)$. These regions were later classified into multiple clusters (H) by mean shift clustering. Each cluster consists of LFOs with unique temporal profiles (I). Granger causality toolbox was used to identify the Granger-cause (J) and Granger-effect $(M)$ in all these clusters. Seed-based $C C M$ at both wavelengths were generated with regard to the Granger-cause (K for $500 \mathrm{~nm}, \mathrm{~L}$ for $700 \mathrm{~nm}$ ) and the Granger-effect (N for $500 \mathrm{~nm}$, O for $700 \mathrm{~nm}$ ). Among all twelve cases with the resection areas inside the optical field of view, $83 \%$ of them showed the Granger-causes in epileptic cortex $(P)$.

Strong LFOs $(<0.1 \mathrm{~Hz})$ were observed in all fourteen acquired data sets. A set of representative LFO analysis results is presented in Fig. 6.3. In this case, negative correlations between $R_{p c a}(p, t)$ at both wavelengths were located in certain focal areas (Fig. 6.3E) . Using K-means segmentation, negative-correlation areas greater than 100 pixels were separated from the remaining cortical areas (Fig. 6.3G). These areas included both epileptic cortex and normal eloquent areas. $\mathrm{R}_{\mathrm{pca}}(\mathrm{p}, \mathrm{t})$ from these isolated areas were 
separated further into multiple clusters by simultaneously using the temporal features identified from both wavelengths (Fig. 6.3H). Each cluster possess a unique oscillation pattern (Fig. 6.3I), for which the spatial distribution could be scattered. For example, Clusters 1 and 2 in Fig. $6.3 \mathrm{H}$ are connected spatially, but the temporal profiles of their $\mathrm{R}_{\mathrm{ci}}(\mathrm{p}, \mathrm{t})$ at both wavelengths (Fig. 6.3I) are clearly different.

To understand effective connections between these epileptic and eloquent areas, the G-causes (Fig. 6.3J) and G-effects (Fig. 6.3M) among the classified clusters were identified using Granger causality method. In general, the clusters located inside the resection area, presumed to be related to seizure onset, were found to be the G-causes. In the cases of the resection area being outside the optical FOV (i.e., below the cortical surface), the eloquent areas could be highlighted after the classification, and they often had a directed influence on other brain areas within the FOV. However, not all the identified clusters exhibited causal relationships (e.g., Cluster 1, 2, and 3 in Fig. 6.3H), which might indicate that these areas were not effectively connected, regardless of their structural or functional connections. Once the G-causes and the G-effects were determined, the averaged $R_{c i}(p, t)$ within a G-cause area and a G-effect area were used as the seeds to obtain CCM based on seed-based correlations with $\mathrm{R}_{\mathrm{pca}}(\mathrm{p}, \mathrm{t})$ of all the other pixels at the corresponding wavelengths. It was clear that the correlated areas revealed at $500 \mathrm{~nm}$ (Fig. 6.3K and 6.3L) are much more focal than those at $700 \mathrm{~nm}$ (Fig. 6.3N and 6.3O), which could be a result of the longer optical path length for biological tissue at $700 \mathrm{~nm}$ discussed in Chapter 4.4. 
Table 6. 2 Analysis Summary

\begin{tabular}{|c|c|c|c|c|c|}
\hline Case & $\begin{array}{l}\mathrm{LF} \\
\mathrm{O}\end{array}$ & $\begin{array}{l}\text { Negative } \\
\text { Correlatio } \\
\mathrm{n}\end{array}$ & $\begin{array}{l}\text { Match with ECoG } \\
\text { and } \\
\text { Resection }\end{array}$ & $\begin{array}{l}\text { Granger- } \\
\text { Causes }\end{array}$ & Comment \\
\hline 1 & $\mathrm{x}$ & $\mathrm{x}$ & $\mathrm{X}$ & $\mathrm{x}$ & \\
\hline $2-1$ & $\mathrm{x}$ & $\mathrm{x}$ & $\mathrm{x}$ & $\mathrm{x}$ & \\
\hline $2-2$ & $\mathrm{x}$ & $\mathrm{X}$ & $\mathrm{x}$ & $\mathrm{x}$ & \\
\hline 3 & $\mathrm{x}$ & $\mathrm{x}$ & $\begin{array}{l}\text { Fast activity and } \\
\text { spike }\end{array}$ & $\begin{array}{l}\text { Fast } \\
\text { activity }\end{array}$ & $\begin{array}{l}\text { Strong LFOs, weakly } \\
\text { anti-correlated, were } \\
\text { found inside the } \\
\text { resection area. They } \\
\text { were correlated to the } \\
\text { area showing fast } \\
\text { activity. }\end{array}$ \\
\hline $4-1$ & $\mathrm{x}$ & $\mathrm{x}$ & $\mathrm{x}$ & $\mathrm{x}$ & \\
\hline $4-2$ & $\mathrm{x}$ & $\mathrm{x}$ & $\mathrm{x}$ & $\mathrm{x}$ & \\
\hline 5 & $\mathrm{x}$ & $\mathrm{x}$ & $\mathrm{x}$ & $\mathrm{x}$ & \\
\hline 6 & $\mathrm{x}$ & $\mathrm{x}$ & Functional & $\begin{array}{l}\text { Functiona } \\
1\end{array}$ & $\begin{array}{l}\text { Resection area was } \\
\text { outside the optical } \\
\text { FOV. }\end{array}$ \\
\hline $7-1$ & $\mathrm{x}$ & $\mathrm{x}$ & Receptive & Receptive & \multirow{2}{*}{$\begin{array}{l}\text { Strong LFOs, } \\
\text { positively correlated, } \\
\text { were found inside the } \\
\text { resection area. }\end{array}$} \\
\hline $7-2$ & $\mathrm{x}$ & $\mathrm{x}$ & Receptive & Receptive & \\
\hline 8 & $\mathrm{x}$ & $\mathrm{x}$ & $\mathrm{x}$ & $\mathrm{x}$ & \\
\hline 9 & $\mathrm{x}$ & $\mathrm{x}$ & $\mathrm{x}$ & $\mathrm{x}$ & \\
\hline 10 & $\mathrm{x}$ & $\mathrm{x}$ & Interictal and motor & Motor & $\begin{array}{l}\text { Resection area was } \\
\text { not within the optical } \\
\text { FOV. }\end{array}$ \\
\hline 11 & $\mathrm{x}$ & $\mathrm{x}$ & $\mathrm{x}$ & $\mathrm{x}$ & \\
\hline
\end{tabular}

LFO: low-frequency oscillation

ECoG: electrocorticography

FOV: field of view

As summarized in Table 6.2, there were two cases in which only eloquent areas were identified within the FOV. For these two cases, the resection areas were either below the cortical surface or adjacent to the craniotomy but covered by skull. Nevertheless, strong hemodynamic LFOs were found within the resection areas (i.e., epileptogenic areas) in all the remaining twelve cases. $75 \%$ of the resection areas exhibited negative correlations 
between their $\mathrm{R}_{\mathrm{pca}}(\mathrm{p}, \mathrm{t})$ at $500 \mathrm{~nm}$ and $700 \mathrm{~nm}$, and they had directed influences on other cortical areas. Meanwhile, $83 \%$ of the G-causes identified by Granger causality method were related to epileptic activities (i.e., both ictal and interictal discharges), as shown in Fig. 6.3P. However, eloquent areas could be the G-cause as well; on some occasions they either directly contributed to the activities in a distant cortex or relayed the activities from epileptic cortex to the distant cortex. This indicates that eloquent cortical areas, if involved, may facilitate the propagation of epileptic activities through their network to remote cortical areas.

\subsubsection{Stochastic modeling and machine learning (Method 2)}

The coupling mechanism between the change in $\mathrm{CBV}$ and that in $\mathrm{SatO}_{2}$ was investigated using an ARX model. The IRFs obtained from the epileptogenic cortex and the eloquent cortex appeared to be different in their temporal profiles. The machine learning method SVM was applied on the training data sets to identify a hyperplane that could identify differences between the IRFs obtained from the epileptogenic areas $(\mathrm{n}=15)$ and those from eloquent areas $(\mathrm{n}=10)$. Subsequently, the accuracy of the differentiation was evaluated by applying the same SVM model to the testing data set. The accuracy of SVM models with ARX of different orders were compared and illustrated in Fig. 6.4A. ARX model at order 11 yielded the highest accuracy (84\%) and largest area under the ROC curve (0.82 out of 1$)$ in SVM. There was no statistically-significant difference between the amplitude of IRFs obtained from the epileptogenic and eloquent cortical areas (Fig. 6.4B). Nevertheless, we observed an optimal hyperplane in SVM that was 
able to separate the IRFs of the epileptogenic cortical areas from those of the eloquent areas with a sensitivity of $93 \%$ and a specificity of $70 \%$ (Fig. 6.4C).
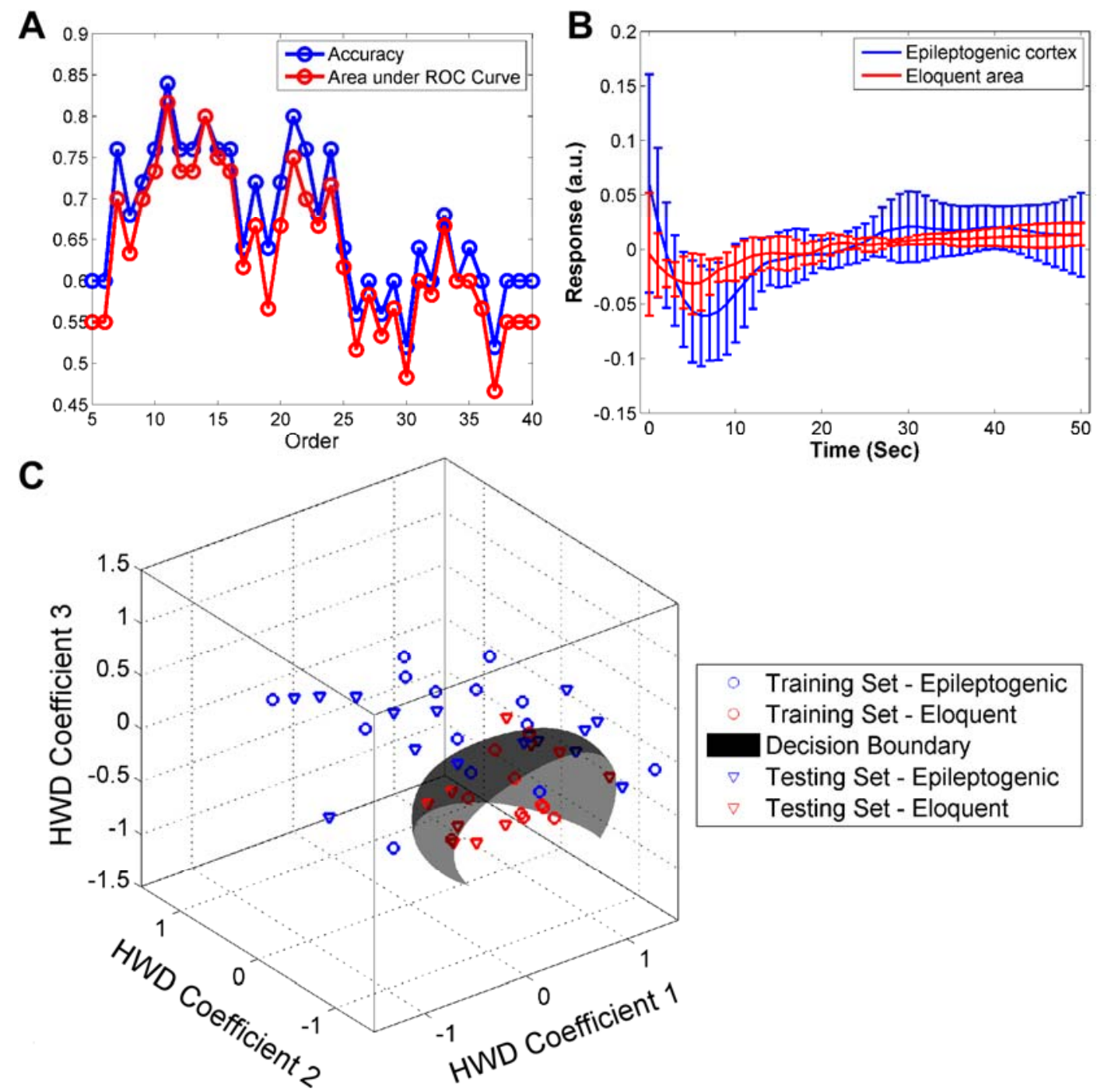

$\begin{array}{ll} & \text { Training Set - Epileptogenic } \\ & \text { Training Set - Eloquent } \\ & \text { Decision Boundary } \\ \nabla & \text { Testing Set - Epileptogenic } \\ \nabla & \text { Testing Set - Eloquent }\end{array}$

Figure 6. 4 Differentiation between epileptogenic and eloquent cortex (Method 2). (A) Autoregressive model with exogenous source $(A R X)$ at Order 11 shows the highest accuracy (0.84) and largest area under receiver operating characteristic (ROC) curve (0.82). (B) The corresponding impulse response functions (IRFs) obtained from epileptogenic (blue) and eloquent (red) areas. (C) The hyperplane obtained by support vector machine (SVM) could separate the epileptogenic cortex from eloquent areas with a sensitivity of $93 \%$ and a specificity of $70 \%(n=25,15$ epileptogenic and 10 eloquent areas). HWD=Haar wavelet decomposition.

\subsubsection{Vasculature abnormalities in the superficial layer (Method 3)}

Because of the image quality and the location of the resection zone, the high resolution cortical surface images from just six patients were used for vasculature 
network characteristic analysis. Representative processed images, which show the edges of blood vessels, are shown in Fig. 6.5A. From the six processed images used, 18 subimages and their histograms were obtained from epileptogenic cortical areas and 20 from random-selected normal areas. Based on the analysis of the histograms of the processed image, it appeared that the density of vessel networks in the superficial layer of normal cortex (Fig. 6.5A, histogram in red) was higher than the one in the epileptogenic cortex (Fig. 6.5A, histogram in blue). To facilitate statistical comparison, each histogram was normalized to its area under the curve. As shown in Fig. 6.5B, a significant difference $(\mathrm{P}<0.05)$ was detected mostly within the low intensity range (3-26) between the normalized histograms obtained from epileptogenic and normal cortical areas, using a two-sample t-test. This suggests that the blood vessel densities in epileptogenic cortical areas may be less than those found in normal areas within the superficial layer. 


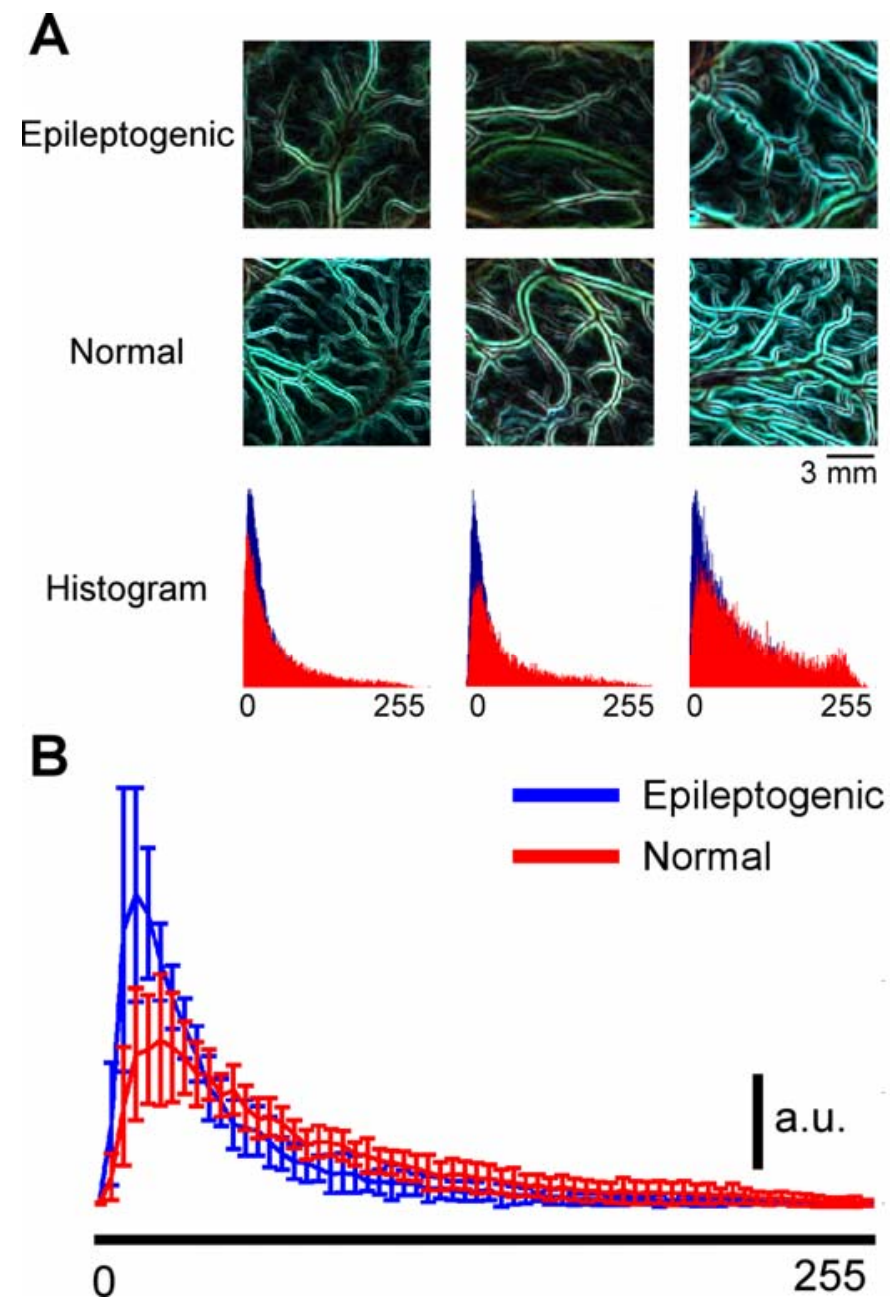

Figure 6. 5 Vasculature abnormalities in the superficial layer (Method 3). A) Examples of vessel networks in epileptogenic and normal cortices and their corresponding histogram (blue: epileptogenic cortex, red: normal cortex). Areas showing normal cortex were randomly selected, regardless of their functional roles. B) Normalized histogram of the vessel network image obtained from the epileptogenic and normal cortices. Six patients (Patient 1, 2, 5, 7, 8, and 9) with high-quality color image of the exposed brain (Number of epileptogenic areas $=18$, number of normal areas $=20$ ). For each patient, the window sizes of the epileptogenic area and normal area were the same. The histograms for each patient were normalized to the highest value in epileptogenic areas' histograms.

\subsection{Discussion}

\subsubsection{Hemodynamic low-frequency oscillations (LFOs)}

The physiological signal centered in the in vivo study reported here are lowfrequency, spontaneous hemodynamic oscillations $(<0.1 \mathrm{~Hz})$. This is an intrinsic property of the live brain because these oscillations do not appear in brains after death [112]. Hemodynamic LFOS have been attributed to the resting-state functional activities 
[103, 112-117]. In the current study, we noticed that LFOs tend to be local and that their localizations were very reproducible from different observations, appearing in eloquent as well as epileptogenic cortical areas. This observation is in agreement with the suggestion by Fox et al that, in addition to normal resting-state functional activities, LFOs may also be the manifestation of the disease-related neural processing [113].

In this study, sophisticated data analysis schemes were employed to differentiate between the LFOs originated from eloquent cortex and those from epileptogenic cortex. Method 1 was used to identify the differences in resting-state hemodynamic LFOs between the eloquent and epileptogenic cortical areas, in terms of temporal correlation, clustering and Granger causality. Instead of relying solely on the magnitudes of LFOs [103], the negative correlation between the DIOI data at $500 \mathrm{~nm}$ and $700 \mathrm{~nm}$ was taken as an indication of underlying neuronal activities, which could be explained by the generally-accepted balloon model $[104,118]$. However, the epileptogenic cortex in two studied patients exhibited a positive correlation between the data at both wavelengths, suggesting that a different neurovascular coupling mechanism might exist. Little effort was devoted to the investigation of the cortical areas with positively-correlated LFOs in the current study, due to the limited number of cases, as well as their similarity to the artifacts caused by specular reflectance. For each studied case, epileptogenic cortex predominantly had directed influences on no less than one area, sometimes in close proximity and sometimes remote, probably because of different propagation mechanisms for the epileptiform discharges. This phenomenon was discussed previously by Song et al. when they studied a preclinical model of focal epilepsy [119]. Such connections were 
further investigated using the G-cause as the reference in a seed-based spatial correlation analysis, which helps us understand the network in a global view and gain knowledge about the different underlying phenomena in the DIOI data. However, even though $83 \%$ of the G-causes were responsible for epileptiform discharges, Method 1 lacks support from physiological-based theories and, hence, is not a good choice for demarcating the resection zone during epilepsy surgery. In addition, Method $\mathbf{1}$ is only applicable when the epileptogenic area is located within the FOV of DIOI, which, in practice, is not always the case. Therefore, Method 2 was devised to address the limitations of Method 1.

\subsubsection{Stochastic modeling to characterize the underlying pathophysiology}

The ARX modeling employed in Method 2 was adopted to identify those unique temporal variations in hemodynamics that occur in epileptogenic cortex during the resting state. SVM based on the outcome of the ARX modeling in Method 2 was very accurate at differentiating epileptogenic cortex from eloquent cortical areas. This success may be attributed to the alterations in the vasculature network, as well as to neurovascular/neurometabolic couplings within the epileptogenic cortex. DIOI at 500 $\mathrm{nm}$ is predominantly used to measure changes in cerebral blood volume (CBV), modulated by activity-evoked dilation of the pial arterioles [51]. On the other hand, DIOI at $700 \mathrm{~nm}$ is sensitive to the variations in the oxygen content of blood (i.e., $\mathrm{SatO}_{2}$ ) within the venous network [51]. Through the ARX modeling of both DIOI signals, the dynamic interplay between the arteriole and venous networks (i.e., vasculature characteristics) was examined. The same approach should also reveal regional neuro-activity and neuro- 
metabolism because of the neurovascular and neurometabolic coupling mechanisms that exist within the capillary bed that forms the bridge between the arteriole and venous networks. From a neurovascular coupling point of view, astrocytes [120], pericytes [121] and endothelium [122] play a critical role in the coupling mechanism. Both pericyte degeneration and thickening of micro-vessel walls have been found in the abnormallyspiking brain areas detected in patients with intractable complex partial epilepsy [123]. In addition, vascular malformations have been frequently identified in association with focal epilepsy [124-126]. Moreover, relative to low-spiking areas, increased vascularity and microglial infiltration have been demonstrated in high-spiking human neocortex [127]. As for alterations in neurometabolic coupling, regional hypometabolism both in cortical and subcortical areas has been demonstrated in preclinical seizure models [128, 129]. Finally, the outcomes of the vasculature analysis (Method 3) identified in this study also reveal significant differences in the vasculature density within the superficial layer between epileptogenic and the normal cortical areas. This being said, the imaging technique used in this study lacked the resolution, contrast and penetration depth to further investigate the exact source of the abnormalities.

\subsubsection{Implications for fMRI techniques}

As discussed above, the relationship between the LFOs obtained by DIOI at $500 \mathrm{~nm}$ and $700 \mathrm{~nm}$ could be used as a biomarker to distinguish the epileptogenic from the normal/eloquent cortex. Because of its limitation in the penetration depth, it is not feasible to use DIOI to detect deep-seated epileptogenic foci. Since fluctuations in DIOI at $500 \mathrm{~nm}$ and $700 \mathrm{~nm}$ are related to the changes in $\mathrm{CBV}$ and $\mathrm{SatO}_{2}$, it is possible to 
detect these physiological characteristics using non-invasive imaging modalities like fMRI. fMRI is capable of measuring blood oxygenation level dependent (BOLD) signals that are associated with regional hemodynamic, as well as metabolic parameters like the oxygen extraction ratio, $\mathrm{CMRO}_{2}, \mathrm{CBF}$ and $\mathrm{CBV}$. In addition, fMRI can measure vascular space occupancy (VASO), which provides indirect access to the changes in CBV associated with neuronal activity [130]. Recently, simultaneous BOLD-fMRI and VASO-fMRI has been performed to study the functionally-induced BOLD and CBV responses in the human brain [131]. Consequently, the same technique therefore can be used to acquire BOLD and CBV signals from the epileptogenic brain in a non-invasive fashion, with data interpreted using the approaches disclosed in Methods $\mathbf{1}$ and 2, thereby allowing for epileptogenic cortex to be identified and separated from normal/eloquent cortex. This combination will be remarkably crucial to the precise detection of deep epileptogenic brain areas during the pre-operation planning phase.

\subsubsection{Consideration of anesthesia}

In this study, all patients were anesthetized by the combination of propofol and fentanyl (Supplementary Table 6.1). Propofol is one of the most popular anesthetic agents used in epilepsy surgery. It is highly recommended for potential use in fMRI studies, since it has relatively minor effects on hemodynamic responses [132] and does not inhibit stimulus-evoked cortical activity in animals or humans [133, 134]. Unfortunately, according to the study by Zijlmans et al., the infusion of propofol alone could produce antiepileptic effect and lead to reduction in certain epileptic activities [135]. Dosedependent propofol alone could also change the cortical and subcortical functional 
connectivity during the resting state [136-138]. Recently, Rayshubskiy et al. [139] reported a DIOI and fMRI study of the brains from two patients. They were able to observe $\sim 0.1 \mathrm{~Hz}$ hemodynamic oscillations in the cortex of the awake patient undergoing brain tumor surgery but not in the patient anesthetized with propofol during epilepsy surgery (note that this epileptic patient was a 36-year-old male, and pathology was not disclosed). The usage of propofol alone might be the reason that they were not able to see hemodynamic LFOs in the epileptogenic cortex. It has also been reported that the propofol has a potential pro-convulsant effect. Cheng et al. [140] suggested that the reported pro-convulsant effects of propofol could be a result of combing propofol with other anesthetic agents such as ketamine and fentanyl. This hypothesis is supported by various studies [141-143]. Based on this evidence, those hemodynamic LFOs observed in eloquent and epileptogenic cortical areas should definitely have their origins in restingstate neuronal activity and epileptiform discharge, respectively.

\subsubsection{Significance and Limitation}

The methods proposed here to identify epileptogenic cortex could be conducted on epilepsy patients under anesthesia during the surgery. They do not require any external stimulation or any reduction in anesthesia to map eloquent areas. DIOI thereby is a promising, complementary tool for intraoperative guidance. It could also provide more specific directions regarding where to place the subdural electrode array for ECoG recordings.

However, due to the limitation of the penetration depth of visible light on biological tissue, DIOI can only investigate the pathophysiological characteristics within the 
superficial layer of the cortex, a limitation that is similar to that of ECoG using the surface electrode arrays [13]. In addition, DIOI is susceptible to artifacts induced by specular reflection because human cortical surfaces are not flat. To overcome these artifacts of specular reflection, the DIOI acquisition could be focused on one area of the exposed cortical surface at a time, from different locations and viewing angles.

Another issue to consider is that this study was conducted on pediatric patients with focal epilepsy, usually accompanied by lesions in the cortex. The feasibility of using the same methods on other types of epilepsy or on different populations remains to be investigated.

\subsection{Conclusion}

In this study, three new analysis approaches to intraoperative imaging were proposed to differentiate epileptogenic from the eloquent cortex in pediatric patients with focal epilepsy. These approaches are based on the causal relationships, the underlying biophysical mechanisms and the vasculature network characteristics of the cortical surface. Together, these methods create a new means of intraoperative epileptogenic cortex localization that is economic and effective at the same time. More importantly, this analysis based on the biophysical mechanisms can differentiate the eloquent area from epileptogenic cortex with high sensitivity and specificity. Neither external stimulation nor reduced anesthesia is required, both of which are required for other novel ifMRI technologies. More importantly, the analytical methods revealed in this study can be applied to data obtained from simultaneous BOLD- and VASO-fMRI, as well as from other non-invasive optical imaging modalities capable of acquiring hemodynamic LFOs 
from the brain. The incorporation of these new methodologies could positively impact the outcome of epilepsy surgery in pediatric patients. 
Supplementary Table 6. 1 Surgery-related Information

\begin{tabular}{|c|c|c|c|c|c|c|c|c|c|c|c|}
\hline Patient & $\begin{array}{l}\text { Imaging } \\
\text { time }\end{array}$ & Propofol, mg & Fentanyl, mg & $\begin{array}{c}\text { Oxyge } \\
n \\
(\mathrm{~L} / \mathrm{min})\end{array}$ & $\begin{array}{c}\text { SEVO } \\
\text { flurane } \\
(\%)\end{array}$ & Temperature & EKG & $\begin{array}{l}\mathrm{FIO}_{2} \\
(\%)\end{array}$ & $\begin{array}{c}\mathrm{SpO}_{2} \\
(\%)\end{array}$ & $\begin{array}{c}\mathrm{CO} \\
2\end{array}$ & PIP \\
\hline 1 & $10: 30 \mathrm{am}$ & $100(7: 30 \mathrm{am})$ & $25(10 \mathrm{am})$ & 2 & 2 & 36 & SR & 50 & 100 & 30 & 18 \\
\hline 2 & $10: 10 \mathrm{am}$ & $\begin{array}{c}110(8 \mathrm{am}), 40(9 \\
\mathrm{am})\end{array}$ & $25(9: 45 \mathrm{am})$ & 1.5 & 2 & 34.7 & SR & 50 & 100 & 27 & 15 \\
\hline 3 & $9: 10 \mathrm{am}$ & na & $10(8: 45 \mathrm{am})$ & 2.5 & 2.5 & 34.6 & SR & 95 & 100 & 29 & 13 \\
\hline 4 & $3: 54 \mathrm{pm}$ & $\begin{array}{c}100(1: 15 \mathrm{pm}), \\
30(2: 30 \mathrm{pm})\end{array}$ & $50(2: 45 \mathrm{pm})$ & 1 & 1.2 & 35.7 & SR & 40 & 100 & 32 & 12 \\
\hline 5 & $9: 22 \mathrm{am}$ & $130(7: 30 \mathrm{am})$ & $\begin{array}{c}50(8: 30 \mathrm{am}, \\
9: 30 \mathrm{am})\end{array}$ & 1 & 1.4 & 36 & SR & 40 & 100 & 33 & 17 \\
\hline 6 & $12: 32 \mathrm{pm}$ & $\begin{array}{l}\text { inf. } 100 \mathrm{mg} / \mathrm{kg} \\
\text { per min }\end{array}$ & $50(12 \mathrm{pm})$ & 1 & 0.5 & 37 & SR & 54 & 100 & 27 & 20 \\
\hline 7 & 1:03 pm & $\begin{array}{c}150(10 \mathrm{am}), 100 \\
(10: 30 \mathrm{am})\end{array}$ & $\begin{array}{c}50(12: 15 \\
\mathrm{pm})\end{array}$ & 1 & 2.5 & 35.5 & SR & 40 & 100 & 36 & 18 \\
\hline 8 & 11:04 am & $\begin{array}{l}\text { inf. } 100 \mathrm{mg} / \mathrm{kg} \\
\text { per min }\end{array}$ & $100(9: 30 \mathrm{am})$ & 2 & na & 35.7 & SR & 50 & 100 & 28 & 16 \\
\hline 9 & $11: 02 \mathrm{am}$ & $100(8: 15 \mathrm{am})$ & $20(9: 45 \mathrm{am})$ & 0.9 & 1.3 & 35 & SR & 35 & 100 & 27 & 16 \\
\hline 10 & $10: 29 \mathrm{am}$ & $\begin{array}{c}\text { inf. } 50 \mathrm{mg} / \mathrm{kg} \text { per } \\
\mathrm{min}\end{array}$ & $50(10 \mathrm{am})$ & 1 & 0.7 & 35.7 & SR & 40 & 100 & 29 & 17 \\
\hline 11 & $3: 00 \mathrm{pm}$ & na & $5(3 \mathrm{PM})$ & 2 & 0.9 & 34.5 & SR & 50 & 100 & 32 & 16 \\
\hline
\end{tabular}

SEVO flurane: Sevoflurane;

EKG: Electrocardiogram;

FIO2: Fraction of inspired oxygen;

$\mathrm{SpO} 2$ : Peripheral capillary oxygen saturation;

PIP: Peak inspiratory pressure;

inf: infusion;

SR: sinus rhythm.

na: not available 


\section{Summary}

In the first part of the dissertation, a new tissue differentiation algorithm was developed to enable intraoperative detection of brain tumors at the resection front using a probe-based diffuse reflectance spectroscopy system. The accuracy of the algorithm was validated experimentally on 20 pediatric patients undergoing brain tumor surgery at Nicklaus Children's Hospital. Three indicative parameters, hemoglobin concentration $([\mathrm{Hb}])$, diffuse reflectance signal at $700 \mathrm{~nm}(\mathrm{Rd} 700)$, and oxygen saturation level $\left(\mathrm{SatO}_{2}\right)$, were extracted from the diffuse reflectance spectra within the visible light region acquired from normal and tumorous brain tissues. Support vector machine (SVM), a machine learning method, was employed to identify the differences between these indicative parameters retrieved from normal brain tissues and from the tumorous tissues, and yielded a very high accuracy (92\%), sensitivity (91\%) and specificity (93\%). These positive results validate the concept of building an intraoperative guidance system for pediatric brain tumor surgery using diffuse reflectance spectroscopy. However, its accuracy could be compromised by the hand motion introduced to the probe.

The second part of the dissertation introduces a novel hybrid spectroscopy imaging system. It was devised to address the two drawbacks of the contact-probe-based diffuse reflectance spectroscopy system in the intraoperative applications: high susceptibility to artifacts induced by hand motion and interference to the surgical procedure. The system was evaluated on its capability to quantify the optical properties of an absorbingscattering medium in a non-contact fashion. The system acquired total diffuse reflectance signals from a subject illuminated by a broad uniform beam, the signals were then 
converted to the ratios of the reduced scattering to the absorption coefficients using a look-up table established by a Monte Carlo simulation model for photon migration. The accuracy of the system was verified theoretically and experimentally over a wide range of optical properties. The system holds tremendous value in in vivo biomedical applications, such as intraoperatively detecting brain tumor at the resection front.

In the third part of the dissertation, a new spectra interpretation algorithm was developed to extract useful hemodynamic and structural characteristics from biological tissue specifically for the newly devised optical system described in the second portion. The algorithm was both validated theoretically with Monte Carlo simulation and tested experimentally with Wistar rats undergoing forepaw stimulation. The new system and algorithm could detect significant changes in all extracted hemodynamic and structural characteristics from the rat somatosensory cortex, which could have tremendous values in both clinical and preclinical applications in the future.

The new system and the spectral interpretation algorithm will be applied in a series of validation studies at Nicklaus Children's Hospital in the near future to ascertain their ability in differentiating brain tumors from normal brain tissue at the resection front. Their performance in terms of detecting tumorous tissues will be compared with that of the contact-probe-based diffuse reflectance spectroscopy system.

The final part of the dissertation introduces three new analytical methods developed to intraoperatively detect and differentiate epileptogenic cortex from eloquent cortex in pediatric patients with focal epilepsy, based on dynamic intrinsic optical imaging (DIOI) and static digital imaging. For each patient, the negatively-correlated hemodynamic lowfrequency oscillations (LFOs, $\sim 0.001-0.01 \mathrm{~Hz}$ ) obtained from the DIOI system at $500 \mathrm{~nm}$ 
and $700 \mathrm{~nm}$ were classified into multiple groups, in accordance with their unique temporal profiles. The causal relationships among these groups were investigated by Granger causality method, and $83 \%$ of the identified epileptogenic cortical areas were found to have a directed influence on one or more cortical areas within the field of view of DIOI. In addition, SVM was employed to differentiate the eloquent cortical area from epileptogenic ones with a high sensitivity (93\%) and adequate specificity (70\%) based on the features extracted from hemodynamic LFOs using the autoregressive modeling with exogenous source and wavelet decomposition methods. This achievement implies unique alterations within the vascular network and/or cellular structure in epileptogenic cortex. A statistically-significant change in vessel density $(\mathrm{P}<0.05)$ indeed was found in the epileptogenic cortices relative to normal areas. These positive results suggest that a new economic and effective means for intraoperative demarcation of epileptogenic cortex may be developed based on DIOI. It has the potential to be used in conjunction with the existing technologies for epileptogenic/eloquent cortex localization to facilitate clinical decision-making. The clinical studies will be extended to a larger population of the pediatric patients with different types of focal epilepsy (i.e., lesional and/or non-lesional), as well as the adult patients. 


\section{LIST OF REFERENCES}

1. Jain RK, di Tomaso E, Duda DG, Loeffler JS, Sorensen AG, Batchelor TT. Angiogenesis in brain tumours. Nat Rev Neurosci. 2007;8(8):610-22. doi: http://www.nature.com/nrn/journal/v8/n8/suppinfo/nrn2175_S1.html.

2. Oliver L, Olivier C, Marhuenda FB, Campone M, Vallette FM. Hypoxia and the malignant glioma microenvironment: regulation and implications for therapy. Curr Mol Pharmacol. 2009;2(3):263-84. Epub 2009/12/22. PubMed PMID: 20021464.

3. Brandão LA, Poussaint TY. Pediatric Brain Tumors. Neuroimaging Clinics of North America. 2013;23(3):499-525.

4. Grondin RT, Scott RM, Smith ER. Pediatric brain tumors. Advances in pediatrics. 2009;56(1):249-69.

5. Ostrom QT, Gittleman H, Liao P, Rouse C, Chen Y, Dowling J, et al. CBTRUS statistical report: primary brain and central nervous system tumors diagnosed in the United States in 2007-2011. Neuro-oncology. 2014;16(suppl 4):iv1-iv63.

6. Fernandez C, Figarella-Branger D, Girard N, Bouvier-Labit C, Gouvernet J, Paredes AP, et al. Pilocytic astrocytomas in children: prognostic factors - a retrospective study of 80 cases. Neurosurgery. 2003;53(3):544-55.

7. Hirsch J-F, Sainte Rose C, Pierre-Kahn A, Pfister A, Hoppe-Hirsch E. Benign astrocytic and oligodendrocytic tumors of the cerebral hemispheres in children. Journal of neurosurgery. 1989;70(4):568-72.

8. Laws Jr ER, Taylor WF, Clifton MB, Okazaki H. Neurosurgical management of low-grade astrocytoma of the cerebral hemispheres. Journal of neurosurgery. 1984;61(4):665-73.

9. Mercuri S, Russo A, Palma L. Hemispheric supratentorial astrocytomas in children: Long-term results in 29 cases. Journal of neurosurgery. 1981;55(2):170-3.

10. Pollack IF, Claassen D, Al-Shboul Q, Janosky JE, Deutsch M. Low-grade gliomas of the cerebral hemispheres in children: an analysis of 71 cases. Journal of neurosurgery. 1995;82(4):536-47.

11. Office of Communications and Public Liaison NIoNDaS. http://www.ninds.nih.gov/disorders/epilepsy/detail_epilepsy.htm\#281563109 2015.

12. Winston GP. Epilepsy surgery, vision, and driving: What has surgery taught us and could modern imaging reduce the risk of visual deficits? Epilepsia. 2013;54(11):1877-88. 
13. Gasser T, Ganslandt O, Sandalcioglu E, Stolke D, Fahlbusch R, Nimsky C. Intraoperative functional MRI: implementation and preliminary experience. Neuroimage. 2005;26(3):685-93.

14. Nabavi A, Black PM, Gering DT, Westin C-F, Mehta V, Pergolizzi Jr RS, et al. Serial intraoperative magnetic resonance imaging of brain shift. Neurosurgery. 2001;48(4):787-98.

15. Gering DT, Weber DM. Intraoperative, real-time, functional MRI. Journal of Magnetic Resonance Imaging. 1998;8(1):254-7.

16. Sommer B, Grummich P, Coras R, Kasper BS, Blumcke I, Hamer HM, et al. Integration of functional neuronavigation and intraoperative MRI in surgery for drugresistant extratemporal epilepsy close to eloquent brain areas. Neurosurgical focus. 2013;34(4):E4.

17. Cannestra AF, Pouratian N, Bookheimer SY, Martin NA, Becker DP, Toga AW. Temporal spatial differences observed by functional MRI and human intraoperative optical imaging. Cerebral Cortex. 2001;11(8):773-82.

18. Unsgaard G, Gronningsaeter A, Ommedal S, Hernes TAN. Brain operations guided by real-time two-dimensional ultrasound: new possibilities as a result of improved image quality. Neurosurgery. 2002;51(2):402-12.

19. El Beltagy MA, Aggag M, Kamal M. Role of intraoperative ultrasound in resection of pediatric brain tumors. Childs Nerv Syst. 2010;26(9):1189-93. Epub 2010/02/25. doi: 10.1007/s00381-010-1091-4. PubMed PMID: 20179947.

20. Gerganov VM, Samii A, Akbarian A, Stieglitz L, Samii M, Fahlbusch R. Reliability of intraoperative high-resolution 2D ultrasound as an alternative to high-field strength MR imaging for tumor resection control: a prospective comparative study. J Neurosurg. 2009;111(3):512-9. Epub 2009/03/31. doi: 10.3171/2009.2.JNS08535. PubMed PMID: 19326992.

21. Auer LM, Velthoven V. Intraoperative ultrasound imaging in neurosurgery: Springer Science \& Business Media; 1990.

22. Knake J, Bowerman R, Silver T, McCracken S. Neurosurgical applications of intraoperative ultrasound. Radiologic Clinics of North America. 1985;23(1):73-90.

23. Keles GE, Lundin DA, Lamborn KR, Chang EF, Ojemann G, Berger MS. Intraoperative subcortical stimulation mapping for hemispheric perirolandic gliomas located within or adjacent to the descending motor pathways: evaluation of morbidity and assessment of functional outcome in 294 patients. Journal of neurosurgery. 2004;100(3):369-75. 
24. Ojemann JG, Ojemann GA, Lettich E. Cortical stimulation mapping of language cortex by using a verb generation task: effects of learning and comparison to mapping based on object naming. Journal of neurosurgery. 2002;97(1):33-8.

25. Signorelli F, Guyotat J, Isnard J, Schneider F, Mohammedi R, Bret P. The value of cortical stimulation applied to the surgery of malignant gliomas in language areas. Neurological Sciences. 2001;22(1):3-10.

26. Tharin S, Golby A. Functional brain mapping and its applications to neurosurgery. Neurosurgery. 2007;60(4):185-202.

27. Bakker Schut T, Witjes M, Sterenborg H, Speelman O, Roodenburg J, Marple E, et al. In vivo detection of dysplastic tissue by Raman spectroscopy. Analytical chemistry. 2000;72(24):6010-8.

28. Harris AT, Rennie A, Waqar-Uddin H, Wheatley SR, Ghosh SK, Martin-Hirsch DP, et al. Raman spectroscopy in head and neck cancer. Head \& neck oncology. 2010;2(1):26.

29. Freeberg JA, Benedet J, MacAulay C, West LA, Follen M. The performance of fluorescence and reflectance spectroscopy for the in vivo diagnosis of cervical neoplasia; point probe versus multispectral approaches. Gynecologic oncology. 2007;107(1):S248S55.

30. Perelman LT. Optical diagnostic technology based on light scattering spectroscopy for early cancer detection. 2006.

31. Brown JQ, Vishwanath K, Palmer GM, Ramanujam N. Advances in quantitative UV-visible spectroscopy for clinical and pre-clinical application in cancer. Current opinion in biotechnology. 2009;20(1):119-31.

32. Bigio IJ, Bown SG. Spectrroscopic sensing of cancer and cancer therapy: Current status of translational research. Cancer biology \& therapy. 2004;3(3):259-67.

33. Cheong W. Summary of optical properties in Optical-Thermal Response of LaserIrradiated Tissue, AJ Welch and MJC van Gemert, Eds., 275-303. New York, NY, Plenum Press; 1995.

34. Prahl SA. Optical absorption of hemoglobin http://omlc.ogi.edu/spectra/hemoglobin/index.html.

35. Wang H-W, Jiang J-K, Lin C-H, Lin J-K, Huang G-J, Yu J-S. Diffuse reflectance spectroscopy detects increased hemoglobin concentration and decreased oxygenation during colon carcinogenesis from normal to malignant tumors. Optics express. 2009;17(4):2805-17. 
36. Mallia R, Thomas SS, Mathews A, Kumar R, Sebastian P, Madhavan J, et al. Oxygenated hemoglobin diffuse reflectance ratio for in vivo detection of oral pre-cancer. Journal of biomedical optics. 2008;13(4):041306--10.

37. Knotzer H, Hasibeder WR. Microcirculatory function monitoring at the bedsidea view from the intensive care. Physiological measurement. 2007;28(9):R65.

38. Verdant C, De Backer D. How monitoring of the microcirculation may help us at the bedside. Current opinion in critical care. 2005;11(3):240-4.

39. Oximeter V. Continuous, noninvasive, and localized microvascular tissue oximetry using visible light spectroscopy. Anesthesiology. 2004;100(6):1469-75.

40. Siegemund M, Van Bommel J, Ince C. Assessment of regional tissue oxygenation. Intensive care medicine. 1999;25(10):1044-60.

41. Toms SA, Lin W-C, Weil RJ, Johnson MD, Jansen ED, Mahadevan-Jansen A. Intraoperative optical spectroscopy identifies infiltrating glioma margins with high sensitivity. Neurosurgery. 2005;57(4):382-91.

42. Lin WC, Toms SA, Johnson M, Jansen ED, Mahadevan-Jansen A. In Vivo Brain Tumor Demarcation Using Optical Spectroscopy $\uparrow$. Photochemistry and Photobiology. 2001;73(4):396-402 .

43. Lin W-C, Sandberg DI, Bhatia S, Johnson M, Oh S, Ragheb J. Diffuse reflectance spectroscopy for in vivo pediatric brain tumor detection. Journal of biomedical optics. 2010;15(6):061709--6.

44. Sun Y, Hatami N, Yee M, Phipps J, Elson DS, Gorin F, et al. Fluorescence lifetime imaging microscopy for brain tumor image-guided surgery. Journal of biomedical optics. 2010;15(5):056022--5.

45. Haglund MM, Berger MS, Hochman DW. Enhanced optical imaging of human gliomas and tumor margins. Neurosurgery. 1996;38(2):308-17.

46. Ti Y, Lin W-C. Effects of probe contact pressure on in vivo optical spectroscopy. Optics express. 2008;16(6):4250-62.

47. Ruderman S, Gomes AJ, Stoyneva V, Rogers JD, Fought AJ, Jovanovic BD, et al. Analysis of pressure, angle and temporal effects on tissue optical properties from polarization-gated spectroscopic probe measurements. Biomedical optics express. 2010;1(2):489-99.

48. Reif R, Amorosino MS, Calabro KW, A'Amar O, Singh SK, Bigio IJ. Analysis of changes in reflectance measurements on biological tissues subjected to different probe pressures. Journal of biomedical optics. 2008;13(1):010502--3. 
49. Lim L, Nichols B, Rajaram N, Tunnell JW. Probe pressure effects on human skin diffuse reflectance and fluorescence spectroscopy measurements. Journal of biomedical optics. 2011;16(1):011012--9.

50. Rivoire K, Nath A, Cox D, Atkinson EN, Richards-Kortum R, Follen M. The effects of repeated spectroscopic pressure measurements on fluorescence intensity in the cervix. American journal of obstetrics and gynecology. 2004;191(5):1606-17.

51. Lavine M, Haglund MM, Hochman DW. Dynamic linear model analysis of optical imaging data acquired from the human neocortex. Journal of neuroscience methods. 2011;199(2):346-62.

52. Cannestra AF, Bookheimer SY, Pouratian N, O'Farrell A, Sicotte N, Martin NA, et al. Temporal and topographical characterization of language cortices using intraoperative optical intrinsic signals. Neuroimage. 2000;12(1):41-54.

53. Sato K, Nariai T, Sasaki S, Yazawa I, Mochida H, Miyakawa N, et al. Intraoperative intrinsic optical imaging of neuronal activity from subdivisions of the human primary somatosensory cortex. Cerebral Cortex. 2002;12(3):269-80.

54. Sobottka SB, Meyer T, Kirsch M, Koch E, Steinmeier R, Morgenstern U, et al. Intraoperative optical imaging of intrinsic signals: a reliable method for visualizing stimulated functional brain areas during surgery: Clinical article. Journal of neurosurgery. 2013;119(4):853-63.

55. Haglund MM, Hochman DW. Optical imaging of epileptiform activity in human neocortex. Epilepsia. 2004;45(s4):43-7.

56. Ma H, Zhao M, Schwartz TH. Dynamic neurovascular coupling and uncoupling during ictal onset, propagation, and termination revealed by simultaneous in vivo optical imaging of neural activity and local blood volume. Cerebral Cortex. 2013;23(4):885-99.

57. Zhao M, Ma H, Suh M, Schwartz TH. Spatiotemporal dynamics of perfusion and oximetry during ictal discharges in the rat neocortex. The Journal of Neuroscience. 2009;29(9):2814-23.

58. Zhao M, Nguyen J, Ma H, Nishimura N, Schaffer CB, Schwartz TH. Preictal and ictal neurovascular and metabolic coupling surrounding a seizure focus. The Journal of Neuroscience. 2011;31(37):13292-300.

59. Song Y, Torres RA, Bae J, Deshmukh A, Lin W-C, Zheng Y, et al. Modeling neuro-vascular/metabolic coupling druing ictal activity in a preclinical model of chronic focal epilepsy: implications on EEG-fMRI techniques. IEEE Transactions on Biomedical Engineering. 2015:Under Review.

60. Wisoff $\mathrm{JH}$, Boyett JM, Berger MS, Brant $\mathrm{C}$, Li H, Yates AJ, et al. Current neurosurgical management and the impact of the extent of resection in the treatment of 
malignant gliomas of childhood: a report of the Children's Cancer Group trial no. CCG945. J Neurosurg. 1998;89(1):52-9. Epub 1998/07/01. doi: 10.3171/jns.1998.89.1.0052. PubMed PMID: 9647172.

61. Levy R, Cox RG, Hader WJ, Myles T, Sutherland GR, Hamilton MG. Application of intraoperative high-field magnetic resonance imaging in pediatric neurosurgery. J Neurosurg Pediatr. 2009;4(5):467-74. Epub 2009/11/03. doi: 10.3171/2009.4.PEDS08464. PubMed PMID: 19877782.

62. Haglund MM, Berger MS, Hochman DW. Enhanced optical imaging of human gliomas and tumor margins. Neurosurgery. 1996;38(2):308-17. Epub 1996/02/01. PubMed PMID: 8869058.

63. Lin WC, Sandberg DI, Bhatia S, Johnson M, Morrison G, Ragheb J. Optical spectroscopy for in-vitro differentiation of pediatric neoplastic and epileptogenic brain lesions. J Biomed Opt. 2009;14(1):014028. Epub 2009/03/05. doi: 10.1117/1.3080144. PubMed PMID: 19256716.

64. Lin WC, Sandberg DI, Bhatia S, Johnson M, Oh S, Ragheb J. Diffuse reflectance spectroscopy for in vivo pediatric brain tumor detection. J Biomed Opt. 2010;15(6):061709. Epub 2011/01/05. doi: 10.1117/1.3505012. PubMed PMID: 21198157.

65. Lin WC, Toms SA, Johnson M, Jansen ED, Mahadevan-Jansen A. In vivo brain tumor demarcation using optical spectroscopy. Photochem Photobiol. 2001;73(4):396402. Epub 2001/05/03. PubMed PMID: 11332035.

66. Toms SA, Lin WC, Weil RJ, Johnson MD, Jansen ED, Mahadevan-Jansen A. Intraoperative optical spectroscopy identifies infiltrating glioma margins with high sensitivity. Neurosurgery. 2007;61(1 Suppl):327-35; discussion 35-6. Epub 2008/09/25. doi: 10.1227/01.neu.0000279226.68751.21. PubMed PMID: 18813157.

67. Chen P, Fernald B, Lin W. Estimation of regional hemoglobin concentration in biological tissues using diffuse reflectance spectroscopy with a novel spectral interpretation algorithm. Phys Med Biol. 2011;56(13):3985-4000. Epub 2011/06/15. doi: 10.1088/0031-9155/56/13/015. PubMed PMID: 21666291.

68. Chen PC, Lin WC. Spectral-profile-based algorithm for hemoglobin oxygen saturation determination from diffuse reflectance spectra. Biomed Opt Express. 2011;2(5):1082-96. Epub 2011/05/12. doi: 10.1364/boe.2.001082. PubMed PMID: 21559121; PubMed Central PMCID: PMC3087566.

69. Mourant JR, Freyer JP, Hielscher AH, Eick AA, Shen D, Johnson TM. Mechanisms of Light Scattering from Biological Cells Relevant to Noninvasive OpticalTissue Diagnostics. Appl Opt. 1998;37(16):3586-93. 
70. Eggert HR, Blazek V. Optical properties of human brain tissue, meninges, and brain tumors in the spectral range of 200 to $900 \mathrm{~nm}$. Neurosurgery. 1987;21(4):459-64. Epub 1987/10/01. PubMed PMID: 3683777.

71. Gebhart SC, Lin WC, Mahadevan-Jansen A. In vitro determination of normal and neoplastic human brain tissue optical properties using inverse adding-doubling. Phys Med Biol. 2006;51(8):2011-27. Epub 2006/04/06. doi: 10.1088/0031-9155/51/8/004. PubMed PMID: 16585842.

72. Taddeucci A, Martelli F, Barilli M, Ferrari M, Zaccanti G. Optical properties of brain tissue. J Biomed Opt. 1996;1(1):117-23.

73. Gevertz JL, Torquato S. Modeling the effects of vasculature evolution on early brain tumor growth. J Theor Biol. 2006;243(4):517-31. Epub 2006/08/30. doi: 10.1016/j.jtbi.2006.07.002. PubMed PMID: 16938311.

74. Holash J, Wiegand SJ, Yancopoulos GD. New model of tumor angiogenesis: dynamic balance between vessel regression and growth mediated by angiopoietins and VEGF. Oncogene. 1999;18(38):5356-62. Epub 1999/09/28. doi: 10.1038/sj.onc.1203035. PubMed PMID: 10498889.

75. Jensen RL, Mumert ML, Gillespie DL, Kinney AY, Schabel MC, Salzman KL. Preoperative dynamic contrast-enhanced MRI correlates with molecular markers of hypoxia and vascularity in specific areas of intratumoral microenvironment and is predictive of patient outcome. Neuro Oncol. 2014;16(2):280-91. Epub 2013/12/07. doi: 10.1093/neuonc/not148. PubMed PMID: 24305704; PubMed Central PMCID: PMC3895375.

76. Law M, Oh S, Johnson G, Babb JS, Zagzag D, Golfinos J, et al. Perfusion magnetic resonance imaging predicts patient outcome as an adjunct to histopathology: a second reference standard in the surgical and nonsurgical treatment of low-grade gliomas. Neurosurgery. 2006;58(6):1099-107; discussion -107. Epub 2006/05/26. doi: 10.1227/01.neu.0000215944.81730.18. PubMed PMID: 16723889.

77. Tietze A, Mouridsen K, Lassen-Ramshad Y, Ostergaard L. Perfusion MRI derived indices of microvascular shunting and flow control correlate with tumor grade and outcome in patients with cerebral glioma. PLoS One. 2015;10(4):e0123044. Epub 2015/04/16. doi: 10.1371/journal.pone.0123044. PubMed PMID: 25875182; PubMed Central PMCID: PMC4395250.

78. Bevilacqua F, Piguet D, Marquet P, Gross JD, Tromberg BJ, Depeursinge C. $<\mathrm{i}>$ In Vivo $</ \mathrm{i}>$ Local Determination of Tissue Optical Properties: Applications to Human Brain. Applied optics. 1999;38(22):4939-50.

79. Cerussi A, Shah N, Hsiang D, Durkin A, Butler J, Tromberg BJ. In vivo absorption, scattering, and physiologic properties of 58 malignant breast tumors 
determined by broadband diffuse optical spectroscopy. Journal of biomedical optics. 2006;11(4):044005--16.

80. Doornbos R, Lang R, Aalders M, Cross F, Sterenborg H. The determination of in vivo human tissue optical properties and absolute chromophore concentrations using spatially resolved steady-state diffuse reflectance spectroscopy. Physics in medicine and biology. 1999;44(4):967.

81. Farrell TJ, Patterson MS, Wilson B. A diffusion theory model of spatially resolved, steady-state diffuse reflectance for the noninvasive determination of tissue optical properties invivo. Medical physics. 1992;19(4):879-88.

82. Fishkin JB, Coquoz O, Anderson ER, Brenner M, Tromberg BJ. Frequencydomain photon migration measurements of normal and malignant tissue optical properties in a human subject. Applied Optics. 1997;36(1):10-20.

83. Matcher S, Cope M, Delpy D. In vivo measurements of the wavelength dependence of tissue-scattering coefficients between 760 and $900 \mathrm{~nm}$ measured with time-resolved spectroscopy. Applied Optics. 1997;36(1):386-96.

84. Tromberg BJ, Shah N, Lanning R, Cerussi A, Espinoza J, Pham T, et al. Noninvasive in vivo characterization of breast tumors using photon migration spectroscopy. Neoplasia. 2000;2(1):26-40.

85. Cuccia DJ, Bevilacqua F, Durkin AJ, Ayers FR, Tromberg BJ. Quantitation and mapping of tissue optical properties using modulated imaging. Journal of biomedical optics. 2009;14(2):024012--13.

86. Bish SF, Rajaram N, Nichols B, Tunnell JW. Development of a noncontact diffuse optical spectroscopy probe for measuring tissue optical properties. Journal of biomedical optics. 2011;16(12):120505-1205053.

87. Foschum F, Kienle A. Broadband absorption spectroscopy of turbid media using a dual step steady-state method. Journal of biomedical optics. 2012;17(3):0370091-7.

88. Song Y, Joasil A, Lin W-C, editors. Optical Spectroscopy for In Vivo Estimation of Hemodynamics and Structural Properties of the Brain. Biomedical Engineering Conference (SBEC), 2013 29th Southern; 2013: IEEE.

89. Wang L, Jacques SL, Zheng L. CONV - convolution for responses to a finite diameter photon beam incident on multi-layered tissues. Computer methods and programs in biomedicine. 1997;54(3):141-50.

90. Wang L, Jacques SL, Zheng L. MCML-Monte Carlo modeling of light transport in multi-layered tissues. Computer methods and programs in biomedicine. 1995;47(2):131-46. 


\section{Prahl SA. http://omlc.org/ prahl/projects/phantoms.html.}

92. Prahl SA, van Gemert MJ, Welch AJ. Determining the optical properties of turbid media by using the adding-doubling method. Applied optics. 1993;32(4):559-68.

93. Moffitt T, Chen Y-C, Prahl SA. Preparation and characterization of polyurethane optical phantoms. Journal of Biomedical Optics. 2006;11(4):041103--10.

94. Jacques SL. Optical properties of biological tissues: a review. Physics in medicine and biology. 2013;58(11):R37.

95. Chen P-C. In vivo tissue diagnosis for myocardial infarction using optical spectroscopy with novel spectral interpretation algorithms. 2011.

96. Chen P-C, Lin W-C. Spectral-profile-based algorithm for hemoglobin oxygen saturation determination from diffuse reflectance spectra. Biomedical optics express. 2011;2(5):1082-96.

97. Paxinos $\mathrm{G}$, Watson $\mathrm{C}$. The rat brain in stereotaxic coordinates: sixth edition: Academic press; 2007.

98. Delpy D, Cope M. Quantification in tissue near-infrared spectroscopy. Philosophical Transactions of the Royal Society B: Biological Sciences. 1997;352(1354):649-59.

99. Dunn AK, Devor A, Dale AM, Boas DA. Spatial extent of oxygen metabolism and hemodynamic changes during functional activation of the rat somatosensory cortex. Neuroimage. 2005;27(2):279-90.

100. Kohl M, Lindauer U, Royl G, Kühl M, Gold L, Villringer A, et al. Physical model for the spectroscopic analysis of cortical intrinsic optical signals. Physics in medicine and biology. 2000;45(12):3749.

101. Leenders K, Perani D, Lammertsma A, Heather J, Buckingham P, Jones T, et al. Cerebral blood flow, blood volume and oxygen utilization normal values and effect of age. Brain. 1990;113(1):27-47.

102. Marchal G, Rioux P, Petit-Taboué M-C, Sette G, Travere J-M, Le Poec C, et al. Regional cerebral oxygen consumption, blood flow, and blood volume in healthy human aging. Archives of neurology. 1992;49(10):1013-20.

103. Song Y, Chen P-C, Bhatia S, Ragheb J, Jayakar P, Lin W-C, editors. Lowfrequency pathophysiological characteristics of pediatric epileptic cortex during the interictal period detected using a dual-wavelength imaging system. SPIE Medical Imaging; 2012: International Society for Optics and Photonics. 
104. Buxton RB. Dynamic models of BOLD contrast. Neuroimage. 2012;62(2):95361.

105. Guo L, Rivero D, Dorado J, Rabunal JR, Pazos A. Automatic epileptic seizure detection in EEGs based on line length feature and artificial neural networks. Journal of neuroscience methods. 2010;191(1):101-9.

106. Subasi A, Gursoy MI. EEG signal classification using PCA, ICA, LDA and support vector machines. Expert Systems with Applications. 2010;37(12):8659-66.

107. Zavar M, Rahati S, Akbarzadeh-T M-R, Ghasemifard H. Evolutionary model selection in a wavelet-based support vector machine for automated seizure detection. Expert Systems with Applications. 2011;38(9):10751-8.

108. Chaplot S, Patnaik L, Jagannathan N. Classification of magnetic resonance brain images using wavelets as input to support vector machine and neural network. Biomedical Signal Processing and Control. 2006;1(1):86-92.

109. El-Naqa I, Yang Y, Wernick MN, Galatsanos NP, Nishikawa RM. A support vector machine approach for detection of microcalcifications. Medical Imaging, IEEE Transactions on. 2002;21(12):1552-63.

110. Ramírez J, Górriz J, Salas-Gonzalez D, Romero A, López M, Álvarez I, et al. Computer-aided diagnosis of Alzheimer's type dementia combining support vector machines and discriminant set of features. Information Sciences. 2013;237:59-72.

111. Mallat SG. A theory for multiresolution signal decomposition: the wavelet representation. Pattern Analysis and Machine Intelligence, IEEE Transactions on. 1989;11(7):674-93.

112. Nasiriavanaki M, Xia J, Wan H, Bauer AQ, Culver JP, Wang LV. High-resolution photoacoustic tomography of resting-state functional connectivity in the mouse brain. Proceedings of the National Academy of Sciences. 2014;111(1):21-6.

113. Fox MD, Raichle ME. Spontaneous fluctuations in brain activity observed with functional magnetic resonance imaging. Nature Reviews Neuroscience. 2007;8(9):70011.

114. Mayhew JE, Askew S, Zheng Y, Porrill J, Westby GM, Redgrave P, et al. Cerebral vasomotion: a $0.1-\mathrm{Hz}$ oscillation in reflected light imaging of neural activity. Neuroimage. 1996;4(3):183-93.

115. Sliwka U, Harscher S, Diehl R, Van Schayck R, Niesen W, Weiller C. Spontaneous oscillations in cerebral blood flow velocity give evidence of different autonomic dysfunctions in various types of headache. Headache: The Journal of Head and Face Pain. 2001;41(2):157-63. 
116. Toronov V, Franceschini MA, Filiaci M, Fantini S, Wolf M, Michalos A, et al. Near-infrared study of fluctuations in cerebral hemodynamics during rest and motor stimulation: temporal analysis and spatial mapping. Medical physics. 2000;27(4):801-15.

117. White BR, Liao SM, Ferradal SL, Inder TE, Culver JP. Bedside optical imaging of occipital resting-state functional connectivity in neonates. Neuroimage. 2012;59(3):2529-38.

118. Buxton RB, Uludağ K, Dubowitz DJ, Liu TT. Modeling the hemodynamic response to brain activation. Neuroimage. 2004;23:S220-S33.

119. Song YS, Basavaraju G ; Hyder, Fahmeed; Lin, Wei-Chiang; Riera, Jorge J. Distributions of irritative zones are related to individual alterations of resting-state networks in focal epilepsy. PLos one. 2015.

120. McCaslin AF, Chen BR, Radosevich AJ, Cauli B, Hillman EM. In vivo 3D morphology of astrocyte-vasculature interactions in the somatosensory cortex: implications for neurovascular coupling. Journal of Cerebral Blood Flow \& Metabolism. 2011;31(3):795-806.

121. Hamilton NB, Attwell D, Hall CN. Pericyte-mediated regulation of capillary diameter: a component of neurovascular coupling in health and disease. Frontiers in neuroenergetics. 2010;2.

122. Chen BR, Kozberg MG, Bouchard MB, Shaik MA, Hillman EM. A critical role for the vascular endothelium in functional neurovascular coupling in the brain. Journal of the American Heart Association. 2014;3(3):e000787.

123. Liwnicz BH, Leach JL, Yeh H-S, Privitera M. Pericyte degeneration and thickening of basement membranes of cerebral microvessels in complex partial seizures: electron microscopic study of surgically removed tissue. Neurosurgery. 1990;26(3):40920.

124. Cui Z, Luan G. A venous malformation accompanying focal cortical dysplasia resulting in a reorganization of language-eloquent areas. Journal of Clinical Neuroscience. 2011;18(3):404-6.

125. Ndode-Ekane X, Hayward N, Gröhn O, Pitkänen A. Vascular changes in epilepsy: functional consequences and association with network plasticity in pilocarpineinduced experimental epilepsy. Neuroscience. 2010;166(1):312-32.

126. Spreafico R, Blümcke I. Focal cortical dysplasias: clinical implication of neuropathological classification systems. Acta neuropathologica. 2010;120(3):359-67.

127. Dachet F, Bagla S, Keren-Aviram G, Morton A, Balan K, Saadat L, et al. Predicting novel histopathological microlesions in human epileptic brain through transcriptional clustering. Brain. 2014:awu350. 
128. Choi H, Kim YK, Kang H, Lee H, Im H-J, Kim EE, et al. Abnormal metabolic connectivity in the pilocarpine-induced epilepsy rat model: a multiscale network analysis based on persistent homology. NeuroImage. 2014;99:226-36.

129. Jupp B, Williams J, Binns D, Hicks RJ, Cardamone L, Jones N, et al. Hypometabolism precedes limbic atrophy and spontaneous recurrent seizures in a rat model of TLE. Epilepsia. 2012;53(7):1233-44.

130. Lu H, Golay X, Pekar JJ, van Zijl P. Functional magnetic resonance imaging based on changes in vascular space occupancy. Magnetic Resonance in Medicine. 2003;50(2):263-74.

131. Krieger SN, Huber L, Poser BA, Turner R, Egan GF. Simultaneous acquisition of cerebral blood volume-, blood flow-, and blood oxygenation-weighted MRI signals at ultra-high magnetic field. Magnetic Resonance in Medicine. 2014.

132. Ding Z, White PF. Anesthesia for electroconvulsive therapy. Anesthesia \& Analgesia. 2002;94(5):1351-64.

133. Kalkman C, Traast H, Zuurmond W, Bovill J. Differential effects of propofol and nitrous oxide on posterior tibial nerve somatosensory cortical evoked potentials during alfentanil anaesthesia. British journal of anaesthesia. 1991;66(4):483-9.

134. Scanley BE, Kennan RP, Cannan S, Skudlarski P, Innis RB, Gore JC. Functional magnetic resonance imaging of median nerve stimulation in rats at $2.0 \mathrm{~T}$. Magnetic resonance in medicine. 1997;37(6):969-72.

135. Zijlmans M, Huiskamp GM, Cremer OL, Ferrier CH, van Huffelen AC, Leijten FS. Epileptic high-frequency oscillations in intraoperative electrocorticography: The effect of propofol. Epilepsia. 2012;53(10):1799-809.

136. Boveroux P, Vanhaudenhuyse A, Bruno M-A, Noirhomme Q, Lauwick S, Luxen A, et al. Breakdown of within-and between-network resting state functional magnetic resonance imaging connectivity during propofol-induced loss of consciousness. Anesthesiology. 2010;113(5):1038-53.

137. Mhuircheartaigh RN, Rosenorn-Lanng D, Wise R, Jbabdi S, Rogers R, Tracey I. Cortical and subcortical connectivity changes during decreasing levels of consciousness in humans: a functional magnetic resonance imaging study using propofol. The Journal of Neuroscience. 2010;30(27):9095-102.

138. Stamatakis EA, Adapa RM, Absalom AR, Menon DK. Changes in resting neural connectivity during propofol sedation. PLoS One. 2010;5(12):e14224.

139. Rayshubskiy A, Wojtasiewicz TJ, Mikell CB, Bouchard MB, Timerman D, Youngerman BE, et al. Direct, intraoperative observation of $0.1 \mathrm{~Hz}$ hemodynamic 
oscillations in awake human cortex: implications for fMRI. Neuroimage. 2014;87:32331.

140. Cheng MA, Tempelhoff R, Silbergeld DL, Theard MA, Haines SK, Miller JW. Large-dose propofol alone in adult epileptic patients: electrocorticographic results. Anesthesia \& Analgesia. 1996;83(1):169-74.

141. Hufnagel A, Elger C, Nadstawek J, Stoeckel H, Böker D. Specific response of the epileptic focus to anesthesia with propofol. Journal of Epilepsy. 1990;3(1):37-45.

142. Wang X, Chen Y, Zhou X, Liu F, Zhang T, Zhang C. Effects of propofol and ketamine as combined anesthesia for electroconvulsive therapy in patients with depressive disorder. The journal of ECT. 2012;28(2):128-32.

143. Yalcin S, Aydoğan H, Selek S, Kucuk A, Yuce HH, Karababa F, et al. Ketofol in electroconvulsive therapy anesthesia: two stones for one bird. Journal of anesthesia. 2012;26(4):562-7. 
VITA

\title{
YINCHEN SONG
}

\author{
Born, Nanjing, China
}

2009

Bachelor of Engineering in Biomedical Engineering Southeast University

China

2009-2015

Graduate Assistant, Research Assistant

Department of Biomedical Engineering

Florida International University

Miami, Florida

\section{PUBLICATION}

Yinchen Song, Sarahy Garcia, Yisel Frometa, Mohamed Almadi, Mohammad Soltani, Jorge J Riera and Wei-Chiang Lin. "A new algorithm for determination of hemodynamic and structural characteristics of brain tissue from total diffuse reflectance measurements". Journal of Biomedical Optics. Submitted. 2015

Po-Ching Chen, Yinchen Song, Sanjiv Bhatia, John Ragheb, Mahlon Johnson, David Sandberg, and Wei-Chiang Lin. "Intraoperative pediatric brain tumor detection using diffuse reflectance spectroscopy". Journal of Biomedical Optics. Submitted. 2015

Yinchen Song, Jorge J Riera, Sanjiv Bhatia, John Ragheb, Claudia Garcia, Alexander G. Weil, Prasanna Jayakar, Wei-Chiang Lin. "Intraoperative optical mapping of epileptogenic cortices in pediatric patients". NeuroImage: Clinical. Under Review. 2015

Yinchen Song, Jessica Ramella-Roman, Mohammad Soltani, Wei-Chiang Lin. "Determination of the optical properties of turbid media using total diffuse reflectance". Biomedical Optics Express. Under Review. 2015

Yinchen Song, Rafael A. Torres, Sarahy Garcia, Yisel Frometa, Jihye Bae, Abhay Deshmukh, Wei-Chiang Lin, Ying Zheng and Jorge J Riera. "Dysfunction of neurovascular/metabolic coupling in chronic focal epilepsy". IEEE Transaction on Biomedical Engineering. In Press. 2015

Yinchen Song, Basavaraju Sanganahalli, Fahmeed Hyder, Wei-Chiang Lin, Jorge J Riera. "Distributions of irritative zones are related to individual alterations of resting-state networks in focal epilepsy". PLOS One 10(7): e0134352. 2015 
Jihye Bae, Abhay Deshmukh, Yinchen Song, Jorge J Riera. "Brain source imaging in rats with preclinical models of focal epilepsy using high resolution EEG recordings". JoVE, (100), e52700, (2015).

Yinchen Song; Arnold Joasil.; Wei-Chiang Lin, "Optical Spectroscopy for In Vivo Estimation of Hemodynamics and Structural Properties of the Brain," Biomedical Engineering Conference (SBEC), 2013 29th Southern, 107-108 (2013).

Nitin, Yadav, Sanjiv Bhatia, John Ragheb, Yinchen Song, Adrian Romero, Sanghoon Oh, Wei-Chiang Lin, "Evaluating and improving the quality of time-dependent, diffuse reflectance spectroscopic signals measured from in vivo brain during craniotomy", Medical Engineering \& Physics, May. 2013.

Yinchen Song, Po-Ching Chen, Sanjiv Bhatia, John Ragheb, Prasanna Jayakar, WeiChiang Lin, "Low-frequency pathophysiological characteristics of pediatric epileptic cortex during the interictal period detected using a dual-wavelength imaging system", Proc. SPIE 8317, 83170V (2012). 